

\title{
Boundary Measures for Symmetric Domains and Integral Formulas for the Discrete Wallach Points
}

\author{
Jonathan Arazy Harald Upmeier
}

\begin{abstract}
Let $D$ be an irreducible hermitian symmetric domain of rank $r$ in $\mathbb{C}^{d}$ and let $G:=$ $A u t(D)$ be the group of all biholomorphic automorphisms of D. We construct explicit integral formulas for the G-invariant inner products on spaces of holomorphic functions on $D$ associated with the discrete Wallach points by means of integration on G-orbits in the boundary $\partial D$ of $D$. These formulas avoid the somewhat unnatural "shifting of parameters" and extend to the infinite dimensional setting of Hilbert-Schmidt symmetric domains. Similar results are obtained, in the bounded and unbounded case, for the "dual" parameters $\alpha_{\ell}$ embedded in the continuous part of the Wallach set. The semi-invariant measures on the boundary orbits are explicitly constructed, including a polar decomposition with respect to a compact subgroup.
\end{abstract}

\section{Contents}

0 Introduction

1 Extension of Invariant Operators on Boundary Orbits of Symmetric Domains

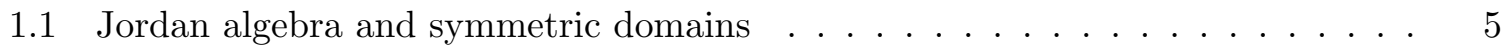

1.2 Invariant differential operators on symmetric cones . . . . . . . . . . . . 9

1.3 Conialization of functions . . . . . . . . . . . . . . . . . 15

1.4 Integration on $K$-orbits $\ldots \ldots \ldots \ldots \ldots \ldots \ldots \ldots \ldots$

2 Integral Formulas for Invariant Inner Products 22

2.1 Discrete Wallach points for Cartan domains . . . . . . . . . . . . . 22

2.2 Discrete Wallach points for symmetric tube domains using Fourier transform . 27

2.3 The case of a symmetric Siegel domain of type $I I \ldots \ldots$. . . . . . . . . 31

2.4 Realization of $\mathcal{H}_{\alpha_{\ell}}(T(\Omega))$ and $\mathcal{H}_{\alpha_{\ell}}(D)$ by boundary integration $\ldots \ldots \ldots \ldots . \quad 41$

3 Semi-invariant Measures on Boundary Orbits 46

3.1 General formulas for homogeneous spaces . . . . . . . . . . . . . 46

3.2 Boundary orbits of symmetric cones . . . . . . . . . . . . . . . 50

*Supported by a grant from the German-Israeli Foundation (GIF), I-415-023.06/95 
3.3 Boundary orbits of symmetric Siegel domains . . . . . . . . . . . . . . 55

3.4 Boundary orbits of Cartan domains . . . . . . . . . . . . . . . . 60

3.5 Restricted boundaries of symmetric domains . . . . . . . . . . . . . . . 69 


\section{Introduction}

For an irreducible hermitian symmetric space $D$ of non-compact type, the holomorphic automorphism group $G=\operatorname{Aut}(D)$ has a (scalar) holomorphic discrete series whose analytic continuation is given by parameters forming the so-called "Wallach set". It is an important problem to give explicit realizations of the corresponding irreducible representations of $G$ in terms of the (boundary) geometry of the underlying domain $D$. A standard reference using Lie theoretic methods is [RV76]. In our previous works ([AU97] and [AU98]) we considered mainly certain parameter values within the continuous part of the Wallach set and constructed realizations emphasizing the Jordan theoretic description of $D$ [FK94]. In this paper we treat the more difficult discrete part and find explicit integral formulas using Lassalle's boundary measures [La87]. The paper contains also a new realization (and proof of existence) of Lassalle's measures, using only basic results from Jordan theory (Peirce decomposition).

The problem of concrete description of the analytic continuation of the holomorphic discrete series by means of Sobolev-type integral formulas attracted the attention of many mathematicians (see for instance [RV76], [O80], [A92-1], [A92-2], [FK90], [Y93], [AU97] and [AU98]). This problem is intimately connected with the problem of the concrete description of the analytic continuation of the Riesz distribution (see [Ri49], [Ga47], [O80], [Gi75] and [AU97]). One of the oldest results on the description of the analytic continuity of the holomorphic discrete series is the realization of $\mathcal{H}_{\frac{d}{r}}$ as the Hardy space $H^{2}(S)=L_{a}^{2}(S, \sigma)$ (where $S$ is the Shilov boundary of $D$ and $\sigma$ is the unique $K$-invariant probability measure on $S$ ).

The shifting method of Yan (see [Y93] and [AU97]) enables one to give integral formulas of the form

$$
<f, g>_{\lambda}=<S_{\lambda, \ell} f, g>_{\lambda+\ell}
$$

for suitable $\ell \in \mathbb{N}$ and shifting operator $S_{\lambda, \ell}$ (which is a $\operatorname{GL}(\Omega)$-invariant differential operator). In particular, if $\lambda+\ell>p-1$ or $\lambda+\ell=\frac{d}{r}$ one obtains integral formulas for $\langle f, g\rangle_{\lambda}$ of the desired type. However, these integral formulas are not best possible, in that

(i) They use unnecessary large numbers of parameters.

(i.e. the topological dimension of the set on which the integration is performed is bigger than the Gelfand-Kirillov dimension of the representation).

(ii) They do not permit generalization to the infinite-rank case.

Our main goal here is to obtain explicit, Sobolev-type integral formulas for the invariant inner products $\left\langle\cdot, \cdot>_{\ell \frac{a}{2}}\right.$ associated with the discrete Wallach points $\ell \frac{a}{2}, \ell=0,1,2, \ldots, r-1$, by means of integration on the $G$-orbits on the boundary $\partial D$. These formulas use the optimal number of parameters (i.e. the topological dimension of the set on which the integration is performed is minimal), and furthermore allow the passage to the case of infinite rank domains. The paper is a continuation of [AU97] and [AU98], in which we develop the formulas of the desired type for $\left\langle f, g>_{\frac{a}{2}}\right.$. The proofs in the general case given here, which are simpler and more conceptual, use the Harish-Chandra isomorphism between the rings of invariant differential operators and the symmetric polynomials.

There is another type of integral formulas for $\langle\cdot, \cdot\rangle_{\lambda}, \lambda \in W(D)$ which use the Cayley transform (which realizes $D$ as a symmetric Siegel domain, denoted by $T(\Omega)$ ) and the Fourier 
transform (which realizes the weighted Bergman spaces on $T(\Omega)$ as weighted $L^{2}$-spaces on $\Omega$ ). These formulas are also extended to the discrete Wallach points $\ell \frac{a}{2}$; they are quite natural but do not allow to work directly with the data coming from $D$.

The paper is organized in the following way. Part 1 is devoted to the construction of the tools needed to prove our results. In Section 1.2 we survey the Harish-Chandra isomorphism between the rings of invariant differential operators on symmetric cones and of the symmetric polynomials. Using spectral theory we extend this result to more general invariant operators. In Section 1.3 we use the conical polar decomposition $Z=K \cdot \Omega$ to study $K$-averaging of certain functions on $D$, (a process we call "conialization"). In Section 1.4 we construct for each $\ell \in\{1,2, \ldots, r-1\}$ two $K$-orbits on $\bar{D}$ and natural measures on them.

After these preparatory sections we prove the above mentioned theorem, in section 1.4 (see Theorem 2.1.7 for the exact formulation). Some related results are established as well.

Section 2.2 is devoted to the development of canonical integral formulas for the inner products $<\cdot, \cdot>_{\ell}, 1 \leq \ell \leq r-1$, in the framework of the symmetric Siegel domain $T(\Omega)$ associated with the Cartan domain $D$ via the Cayley transform. The case of symmetric Siegel domains of type I (i.e. tubes over the symmetric cones $\Omega$ ) is treated first, where we use in an essential way the semi-invariant Lassalle measures on the boundary orbits $\partial_{\ell} \Omega$ of the cone $\Omega$. The development of the analogous integral formulas in the context of symmetric Siegel domains of type II is technically harder and requires additional efforts. In Section 2.4 we use the Lassalle measures to construct integral formulas for the invariant inner products associated with the continuous Wallach points $\alpha_{\ell}:=\frac{d}{r}+\ell \frac{a}{2}, 0 \leq \ell \leq r-1$, for symmetric Siegel domains of type II, which generalize the analogous formulas for symmetric Siegel domains of type I constructed in [AU97].

Finally, in Part 3 we present a new construction of the Lassalle measures. Unlike the original construction of Lassalle (see [La87]) which uses local coordinates (coming from the subgroup $A N$ of $G L(\Omega)$ ), our formulas use global coordinates and make the semi-invariance apparent. 


\section{Extension of Invariant Operators on Boundary Orbits of Symmetric Domains}

\subsection{Jordan algebra and symmetric domains}

In this section we review some known results in analysis on Jordan algebras and triples and on the associated symmetric domains, and establish the notation. For more information see [Hu63], [Gi64], [Lo77], [U87], [FK94] and [A95].

Let $D \subset \mathbb{C}^{d}$ be a Cartan domain, i.e. $D$ is an irreducible bounded symmetric domain in the Harish-Chandra realization. This is equivalent to saying that $D$ is the open unit ball of $\mathbb{C}^{d}$ with respect to a certain norm $\|\cdot\|$, such that the group $G:=\operatorname{Aut}(D)$ of all biholomorphic automorphisms of $D$ acts transitively on $D$. By [Lo77], [U87], there exists a triple product $\{\cdot, \cdot, \cdot\}: \mathbb{C}^{d} \times \mathbb{C}^{d} \times \mathbb{C}^{d} \rightarrow \mathbb{C}^{d}$ so that $Z:=\left(\mathbb{C}^{d},\|\cdot\|,\{\cdot, \cdot, \cdot\}\right)$ is a Jordan-Banach ${ }^{*}$-triple (JB*triple). The maximal compact subgroup of $G$ is $K:=\{\varphi \in G ; \varphi(0)=0\}=G \cap \operatorname{GL}(Z)$, and $D \equiv G / K$.

Let $(r, a, b)$ be the type of $D$ (or, of $Z$ ), where $r$ is the rank and $a, b$ are the characteristic multiplicities. Thus the dimension $d$ and the genus $p$ are given by

$$
d=r+\frac{r(r-1)}{2} a+r b, \quad p=2+(r-1) a+b .
$$

A tripotent $v \in Z$ is an element satisfying $\{v, v, v\}=v$. The Peirce decomposition associated with the tripotent $v$ is

$$
Z=Z_{1}(v) \oplus Z_{\frac{1}{2}}(v) \oplus Z_{0}(v)
$$

where $Z_{\nu}(v):=\{z \in Z ;\{v, v, z\}=\nu z\}, \quad \nu=1, \frac{1}{2}, 0$. The associated Peirce projection $P_{\nu}(v)$, is the projection whose range is $Z_{\nu}(v)$ and whose kernel is the sum of the other two Peirce subspaces. We denote also

$$
D_{\nu}(v):=D \cap Z_{\nu}(v) .
$$

The spaces $Z_{\nu}(v)$ are sub-triples of $Z$, and the rank of the tripotent $v$ is by definition the rank of $Z_{1}(v)$. We define

$$
S_{j}=\text { the set of tripotents of } \operatorname{rank} j, \quad j=0,1,2, \ldots, r .
$$

$S:=S_{r}$ is the Shilov boundary of $D$. Let us choose a frame

$$
e_{1}, e_{2}, \ldots, e_{r}
$$

i.e. a maximal set of tripotents of rank one which are pairwise orthogonal, i.e. $\left\{e_{i}, e_{i}, e_{j}\right\}=0$ whenever $i \neq j$. The tripotent

$$
e=e_{1}+e_{2}+\ldots+e_{r}
$$

is maximal (having rank $r$ ), and thus $Z_{0}(e)=0$. The stabilizer of $e$ in $K$, namely

$$
L:=\{k \in K ; k(e)=e\},
$$

will play an important role in the sequel. Notice that since $K$ acts transitively on $S$, we have $S \equiv K / L$. More generally, $K$ acts transitively on the frames, and in particular it is transitive 
on each of the $S_{j}$. The sub-triple $Z_{1}(e)$ has the structure of a $J B^{*}$-algebra with respect to the product $z \circ w:=\{z, e, w\}$ and the involution $z^{*}:=\{e, z, e\}$, and $e$ is the unit of $Z_{1}(e)$. The real part of $Z_{1}(e)$, i.e. the subset $X=X_{1}(e):=\left\{x \in Z_{1}(e) ; x^{*}=x\right\}$ of self-adjoint elements of $Z_{1}(e)$ is a Euclidean (or formally-real) Jordan algebra, with determinant ("norm") and trace polynomials

$$
N(z)=\operatorname{det}(z) \text { and } \operatorname{tr}(z):=<z, e>
$$

respectively. Here $\langle z, w\rangle$ denotes the unique $K$-invariant scalar product on $Z$ satisfying $\left\langle e_{1}, e_{1}\right\rangle=$ 1. The set

$$
\Omega:=\left\{x^{2} ; x \in X, N(x) \neq 0\right\}
$$

is the symmetric cone associated with $X$. The group $L$, restricted to $X$, coincides with the Jordan-algebra automorphisms of $X$. In particular, it is transitive on the frames of orthogonal minimal idempotents in $X$ whose sum is the unit element $e$.

For $1 \leq j \leq r$, let $u_{j}=e_{1}+\ldots+e_{j}$ and let $N_{j}$ denote the determinant polynomial of the Jordan sub-algebra $Z^{(j)}:=Z_{1}\left(u_{j}\right)$, extended to all of $Z$ via $N_{j}(z):=N_{j}\left(P_{1}\left(u_{j}\right) z\right)$. Note that $N_{r} \equiv N$. The conical function associated with $\mathbf{s}=\left(s_{1}, s_{2}, \ldots, s_{r}\right) \in \mathbb{C}^{r}$ is defined by

$$
N_{\mathbf{S}}(x):=N_{1}(x)^{s_{1}-s_{2}} N_{2}(x)^{s_{2}-s_{3}} \cdots N_{r-1}(x)^{s_{r-1}-s_{r}} N_{r}(x)^{s_{r}}, \quad \forall x \in \Omega .
$$

A partition is a sequence $\mathbf{m}=\left(m_{1}, m_{2}, \ldots, m_{r}\right)$ of integers so that $m_{1} \geq m_{2} \geq \ldots \geq m_{r} \geq 0$. Note that for any partition $\mathbf{m}, N_{\mathbf{m}}$ is a polynomial (called conical), and it extends to all of $Z$. Let us denote

$$
P_{\mathbf{m}}:=\operatorname{span}\left\{N_{\mathbf{m}} \circ k ; k \in K\right\} .
$$

A fundamental theorem [Sch69], (see also [U86]) says that the spaces $P_{\mathbf{m}}$ are irreducible and mutually inequivalent with respect to the action $\pi(k)(f):=f \circ k^{-1}$ of $K$, and that the space $\mathcal{P}$ of all holomorphic polynomials on $Z$ is their direct sum: $\mathcal{P}=\sum_{\mathbf{m}}^{\oplus} P_{\mathbf{m}}$. Thus the $P_{\mathbf{m}}$ are mutually orthogonal with respect to any $K$-invariant inner-product on $\mathcal{P}$. The Fischer-Fock inner-product on $\mathcal{P}$ is given by

$$
<f, g>_{F}=\frac{1}{\pi^{d}} \int_{\mathbb{C}^{d}} f(z) \overline{g(z)} e^{-|z|^{2}} d m(z),
$$

where $|\cdot|$ is the Euclidean norm, and $d m(z)$ is the Lebesgue measure. The reproducing kernel of $P_{\mathbf{m}}$ with respect to $\langle\cdot, \cdot\rangle_{F}$ is denoted by $K_{\mathbf{m}}(z, w)$. Thus, $\sum_{\mathbf{m}} K_{\mathbf{m}}(z, w)=e^{<z, w>}$.

The Gindikin-Koecher Gamma function associated with the cone $\Omega$ is defined for $\mathbf{s}=$ $\left(s_{1}, s_{2}, \ldots, s_{r}\right) \in \mathbb{C}^{r}$ with $\Re s_{j}>(j-1) \frac{a}{2}$ by the convergent integral

$$
\Gamma_{\Omega}(\mathbf{s}):=\int_{\Omega} e^{-t r(x)} N_{\mathbf{s}}(x) d \mu_{\Omega}(x),
$$

where $d \mu_{\Omega}(x):=N(x)^{-\frac{d_{1}}{r}} d m(x)$ is the (unique up to a multiplicative constant) measure on $\Omega$ which is invariant under the group $\operatorname{GL}(\Omega):=\{g \in \mathrm{GL}(X) ; g(\Omega)=\Omega\}$, and $d_{1}:=\operatorname{dim}_{\mathbb{R}}(X)=$ $\frac{r(r-1)}{2} a+r$. It is known that $\Gamma_{\Omega}$ can be expressed as a product of ordinary Gamma functions:

$$
\Gamma_{\Omega}(\mathbf{s}):=(2 \pi)^{\frac{d_{1}-r}{2}} \prod_{j=1}^{r} \Gamma\left(s_{j}-(j-1) \frac{a}{2}\right),
$$


and this allows the extension of $\Gamma_{\Omega}$ to a meromorphic function on all of $\mathbb{C}^{r}$. The Beta function associated with the cone $\Omega$ is related to the Gamma function via

$$
\mathrm{B}_{\Omega}(\mathbf{p}, \mathbf{q}):=\frac{\Gamma_{\Omega}(\mathbf{p}) \Gamma_{\Omega}(\mathbf{q})}{\Gamma_{\Omega}(\mathbf{p}+\mathbf{q})} .
$$

For $\lambda \in \mathbb{C}$ and any partition $\mathbf{m}$ we denote

$$
(\lambda)_{\mathbf{m}}:=\frac{\Gamma_{\Omega}(\lambda+\mathbf{m})}{\Gamma_{\Omega}(\lambda)}=\prod_{j=1}^{r}\left(\lambda-(j-1) \frac{a}{2}\right)_{m_{j}},
$$

where $(t)_{m}:=t(t+1)(t+2) \cdots(t+m-1)$.

Let $h(z, w)$ be the unique $K$-invariant irreducible polynomial, which in holomorphic in $z$, anti-holomorphic in $w$, and satisfies $h(x, x)=N\left(e-x^{2}\right) \quad \forall x \in X$. It is known that

$$
h(z, w)^{-\lambda}=\sum_{\mathbf{m}}(\lambda)_{\mathbf{m}} K_{\mathbf{m}}(z, w), \quad \forall z, w \in D, \quad \forall \lambda \in \mathbb{C},
$$

and the series converges absolutely and uniformly on compact subsets of $D \times D \times \mathbb{C}$. The fundamental formula (1.1.16) (called the "binomial expansion") was proved in special cases in [Hu63] and [La86], and in full generality in [FK94]. The Wallach set $W(D)$ of $D$ consists of all those $\lambda \in \mathbb{C}$ for which $(z, w) \mapsto h(z, w)^{-\lambda}$ is positive definite. Using the expansion (1.1.16) one sees that

$$
W(D)=\left\{0, \frac{a}{2}, 2 \frac{a}{2}, \ldots,(r-1) \frac{a}{2}\right\} \cup\left((r-1) \frac{a}{2}, \infty\right) .
$$

This result was established by several authors using various techniques: [Be75], [RV76] (in the context of Siegel domains), [W79], [La87] and [FK90]. For each $\lambda \in W(D)$ we denote by $\mathcal{H}_{\lambda}$ the completion of $\operatorname{span}\left\{h(\cdot, w)^{-\lambda} ; w \in D\right\}$ with respect to the unique inner-product $\left\langle\cdot, \cdot>_{\lambda}\right.$ determined by

$$
<h(\cdot, w)^{-\lambda}, h(\cdot, z)^{-\lambda}>_{\lambda}=h(z, w)^{-\lambda}, \quad \forall z, w \in D .
$$

Point evaluations are continuous linear functionals on $\mathcal{H}_{\lambda}$ and the corresponding reproducing kernel is $h(z, w)^{-\lambda}$.

If $\lambda>(r-1) \frac{a}{2}$ then $\mathcal{H}_{\lambda}$ contains $\mathcal{P}$ as a dense subspace. On the other hand, for the discrete Wallach points (which are our main concern in this paper) $\ell \frac{a}{2}, 0 \leq \ell \leq r-1, \mathcal{H}_{\ell \frac{a}{2}}$ is the completion of

$$
\mathcal{P}_{\ell}:=\sum_{m_{1} \geq \cdots m_{\ell} \geq 0=m_{\ell+1}=\cdots=m_{r}} P_{\mathbf{m}} .
$$

Since $K$ acts irreducibly on each $P_{\mathbf{m}}$, every $K$-invariant inner product on $P_{\mathbf{m}}$ is proportional to the Fischer inner product. The computation of the proportionality constants for the inner products $\langle\cdot, \cdot\rangle_{\lambda}$ is one of the major steps in the proof of (1.1.16). Thus for every $\lambda \in W(D)$ and every partition $\mathbf{m}$ for which $P_{\mathbf{m}} \subset \mathcal{H}_{\lambda}$,

$$
<f, g>_{\lambda}=\frac{<f, g>_{F}}{(\lambda)_{\mathbf{m}}}, \quad \forall f, g \in P_{\mathbf{m}} .
$$

This implies for all functions $f=\sum_{\mathbf{m}} f_{\mathbf{m}}$ and $g=\sum_{\mathbf{m}} g_{\mathbf{m}}$ in $\mathcal{H}_{\lambda}$ (with $f_{\mathbf{m}}, g_{\mathbf{m}} \in P_{\mathbf{m}} \forall \mathbf{m}$ ),

$$
<f, g>_{\lambda}=\sum_{\mathbf{m}} \frac{<f_{\mathbf{m}}, g_{\mathbf{m}}>_{F}}{(\lambda)_{\mathbf{m}}} .
$$


Let us define an action of $G$ on functions on $D$ via

$$
\left(U^{(\lambda)}\left(\varphi^{-1}\right) f\right)(z):=f(\varphi(z))(J \varphi(z))^{\frac{\lambda}{p}}, \quad \varphi \in G,
$$

where $J \varphi(z):=\operatorname{Det}\left(\varphi^{\prime}(z)\right)$. Then, for $\lambda \in W(D), U^{(\lambda)}$ is a projective representation of $G$ on $\mathcal{H}_{\lambda}$.

It is well known that for $\lambda>p-1 \mathcal{H}_{\lambda}$ is the weighted Bergman space $L_{a}^{2}\left(D, \mu_{\lambda}\right)$, i.e. the space of all analytic functions in $L^{2}\left(D, \mu_{\lambda}\right)$, where

$$
d \mu_{\lambda}(z):=c_{\lambda} h(z, z)^{\lambda-p} d m(z), \quad c_{\lambda}:=\frac{\Gamma_{\Omega}(\lambda)}{\pi^{d} \Gamma_{\Omega}\left(\lambda-\frac{d}{r}\right)} .
$$

The representations $\left\{U^{(\lambda)} ; \lambda>p-1\right\}$ form the holomorphic discrete series of representations of $G$.

We now turn to the structure of the boundary $\partial D$ and introduce some more notation. The boundary component associated to a tripotent $v$ is the set $B(v):=v+D_{0}(v)$ (see (1.1.3)). Its closure is a face of $\bar{D}$ and all the faces arise in this way. Notice that $D_{1}(v)$ and $D_{0}(v)$ are Cartan domains of type $(\ell, a, 0)$ and $(r-\ell, a, b)$ respectively, where $\ell:=\operatorname{rank}(v)$. Let us denote

$$
\partial_{\ell} D:=\cup_{v \in S_{\ell}} B(v), \quad 1 \leq \ell \leq r .
$$

The sets $\partial_{\ell} D$ are the $G$-orbits on $\partial D$, and:

$$
\partial_{\ell} D=G\left(u_{\ell}\right)=\left\{\varphi\left(u_{\ell}\right) ; \varphi \in G\right\},
$$

where $\left.\left\{e_{j}\right\}_{j=1}^{r}\right)$ is the fixed frame and $u_{\ell}=e_{1}+\cdots+e_{\ell}$. Thus

$$
\partial D=\cup_{\ell=1}^{r} \partial_{\ell} D
$$

and the orbits of $G$ in $\bar{D}$ are $\partial_{0} D:=D, \partial_{1} D, \ldots$, and $\partial_{r} D=S$. Let us denote also

$$
v_{\ell}=e-u_{\ell}=e_{\ell+1}+\cdots+e_{r}
$$

Then $u_{\ell}, v_{\ell}$ are orthogonal tripotents of rank $\ell$ and $r-\ell$ respectively, and $u_{\ell}+v_{\ell}=e . Z^{(\ell)}:=$ $Z_{1}\left(u_{\ell}\right)$ is a $\mathrm{JB}^{*}$-sub-algebra of $Z$ with unit $u_{\ell}$, real part

$$
X^{(\ell)}:=\left\{z \in Z^{(\ell)} ; z^{*}=z\right\},
$$

and associated symmetric cone

$$
\Omega^{(\ell)}:=\left\{x^{2} ; x \in X^{(\ell)}, N_{\ell}(x) \neq 0\right\} .
$$

Let $T \in \mathcal{D}_{\ell}$ be extended to a $K^{(\ell)^{\mathbb{C}}}$-invariant differential operator on $Z_{0}\left(v_{\ell}\right)$. Given a tripotent $v \in S_{r-l}$, we define a differential operator $T_{v}$ on $Z_{0}(v)$ in the following way. Since $K$ acts transitively on $S_{r-\ell}$, there exists $k \in K$ for which $k\left(v_{\ell}\right)=v$. We define

$$
T_{v}:=C_{k}^{-1} T C_{k}
$$


where $C_{k}(f):=f \circ k . T_{v}$ is well-defined, i.e. independent of the particular $k \in K$ for which $k\left(v_{\ell}\right)=v$. Indeed, if $k_{1}, k_{2} \in K$ satisfy $k_{1}\left(v_{\ell}\right)=k_{2}\left(v_{\ell}\right)=v$, then $k_{1}^{-1}\left(k_{2}\left(v_{\ell}\right)\right)=v_{\ell}$, and so $k_{2}=k_{1} k$ for some $k \in K$ for which $k\left(v_{\ell}\right)=v_{\ell}$. As we remarked above, $k \in K^{(\ell)}$, and therefore

$$
C_{k_{2}} T C_{k_{2}}=C_{k_{1}}^{-1} C_{k}^{-1} T C_{k} C_{k_{1}}=C_{k_{1}} T C_{k_{1}} \text {. }
$$

For any function $f$ on $\bar{D}$ and any tripotent $v$, the restriction of $f$ to $B(v)$ yields a function $f_{v}$ on $D_{0}(v)$ via

$$
f_{v}(z):=f(v+z), \quad z \in D_{0}(v) .
$$

For any $1 \leq \ell \leq r$ let $\nu_{\ell}$ be the unique $K$-invariant probability measure on $S_{\ell}$, defined via

$$
\int_{S_{\ell}} f d \nu_{\ell}:=\int_{K} f\left(k\left(u_{\ell}\right)\right) d k
$$

Our main result in this framework is the following theorem (compare Theorem 2.1.7)

Theorem Let $1 \leq \ell \leq r-1$ and let $\lambda>(\ell-1) \frac{a}{2}$. Then there exists $T=T^{(\ell, \lambda)} \in \mathcal{D}_{\ell}$ so that for every $f, g \in \mathcal{H}_{\ell \frac{a}{2}}$ which are analytic in a neighborhood of $\bar{D}$,

$$
<f, g>_{\ell \frac{a}{2}}=\int_{S_{r-\ell}}<T_{v} f_{v}, g_{v}>_{\mathcal{H}_{\lambda}\left(D_{0}(v)\right)} d \nu_{r-\ell}(v) .
$$

For general $f, g \in \mathcal{H}_{\ell \frac{a}{2}}$ the integral (1.1.29) is an improper Riemann integral, namely

$$
<f, g>_{\ell \frac{a}{2}}=\lim _{t \nearrow 1} \int_{S_{r-\ell}}<T_{v}\left(f^{t}\right)_{v},\left(g^{t}\right)_{v}>_{\mathcal{H}_{\lambda}\left(D_{0}(v)\right)} d \nu_{r-\ell}(v)
$$

where $f^{t}(z):=f(t z), g^{t}(z):=g(t z)$.

We remark that the case $\ell=0$ in the above theorem (and in subsequent results) is trivial since $\mathcal{H}_{0}$ consists of constant functions.

\subsection{Invariant differential operators on symmetric cones}

In this section we review briefly the connection between the $\operatorname{ring} \mathcal{D}=\operatorname{Diff}(\Omega) \operatorname{GL}(\Omega)$ of $\mathrm{GL}(\Omega)$ invariant differential operators on $\Omega$ and the ring $\mathcal{S}$ of symmetric polynomials in $r$ variables. See [FK94] for more details and [He78] for the general theory.

We denote the half-sum of the strongly orthogonal positive roots by

$$
\boldsymbol{\rho}=\left(\rho_{1}, \rho_{2}, \ldots, \rho_{r}\right) \text { where } \rho_{j}:=(2 j-r-1) \frac{a}{4}, 1 \leq j \leq r .
$$

The $L$-spherical functions are the $L$-averages of the conical functions:

$$
\Phi_{\boldsymbol{\lambda}}(x):=\int_{L} N_{\boldsymbol{\lambda}}(\ell(x)) d \ell .
$$

They are $L$-invariant and normalized by the condition $\Phi_{\boldsymbol{\lambda}}(e)=1$. It is known that the $\Phi_{\boldsymbol{\lambda}}$ are the spherical functions associated with the Riemannian symmetric space $\Omega$ in the usual sense. 
The Weyl group $W_{r}$ in this case is simply the permutation group, acting naturally on $\mathbb{C}^{r}$ and thus on the $\Phi_{\boldsymbol{\lambda}}$ 's. It is known that $\Phi_{\boldsymbol{\lambda}}=\Phi_{\boldsymbol{\mu}}$ if and only if $\boldsymbol{\lambda}$ and $\boldsymbol{\mu}$ are in the same orbit of $W_{r}$.

For each partition $\mathbf{m}$ the function $\Phi_{\mathbf{m}}$ is an $L$-invariant polynomial which belongs to $P_{\mathbf{m}}$ and in particular extends to a polynomial on $Z$. Every $L$-invariant polynomial in $P_{\mathbf{m}}$ is proportional to $\Phi_{\mathbf{m}}$. The ring

$$
\mathcal{S}=\mathbb{C}\left[\lambda_{1}, \lambda_{2}, \ldots, \lambda_{r}\right]^{W_{r}}
$$

of symmetric (i.e. permutation invariant) polynomials in $\boldsymbol{\lambda}=\left(\lambda_{1}, \lambda_{2}, \ldots, \lambda_{r}\right)$ is isomorphic to the full polynomial ring $\mathbb{C}\left[\sigma_{1}, \sigma_{2}, \ldots, \sigma_{r}\right]$ via the elementary symmetric polynomials $\left\{\sigma_{j}\right\}_{j=1}^{r}$ defined by

$$
\sigma_{j}(\boldsymbol{\lambda}):=\sum_{1 \leq i_{1}<i_{2}<\cdots<i_{j} \leq r} \lambda_{i_{1}} \lambda_{i_{2}} \cdots \lambda_{i_{j}} .
$$

Thus, for each $p \in \mathcal{S}$ there is a unique polynomial $q \in \mathbb{C}\left[\sigma_{1}, \sigma_{2}, \ldots, \sigma_{r}\right]$ so that

$$
p(\boldsymbol{\lambda})=q\left(\sigma_{1}(\boldsymbol{\lambda}), \sigma_{2}(\boldsymbol{\lambda}), \ldots, \sigma_{r}(\boldsymbol{\lambda})\right) .
$$

Thus, $\left\{\sigma_{j}\right\}_{j=1}^{r}$ are algebraically independent generators of $\mathcal{S}$.

A fundamental property of the spherical functions is that they are the joint eigenfunctions of the operators in $\mathcal{D}$.

Theorem 1.2.1 (i) The conical and the spherical functions are eigenfunctions of every $T \in \mathcal{D}:$ For all $\boldsymbol{\lambda} \in \mathbb{C}^{r}$ we have

$$
T\left(N_{\boldsymbol{\lambda}+\boldsymbol{\rho}}\right)=\gamma_{T}(\boldsymbol{\lambda}) N_{\boldsymbol{\lambda}+\boldsymbol{\rho}}, \quad T\left(\Phi_{\boldsymbol{\lambda}+\boldsymbol{\rho}}\right)=\gamma_{T}(\boldsymbol{\lambda}) \Phi_{\boldsymbol{\lambda}+\boldsymbol{\rho}}
$$

(ii) $\gamma_{T}(\boldsymbol{\lambda})$ is a symmetric polynomial in $\lambda_{1},, \lambda_{2}, \ldots, \lambda_{r}$, thus $\gamma_{T} \in \mathcal{S}$.

(iii) The map $\gamma: \mathcal{D} \rightarrow \mathcal{S}$ defined via $\mathcal{D} \ni T \mapsto \gamma_{T} \in \mathcal{S}$ is a surjective ring isomorphism, called the Harish-Chandra isomorphism.

(iv) $\mathcal{D}$ is commutative.

Definition 1.2.2 For $1 \leq j \leq r$ we define $\Delta_{j}:=\gamma^{-1}\left(\sigma_{j}\right)$. Namely, for every $\boldsymbol{\lambda} \in \mathbb{C}^{r}$ :

$$
\Delta_{j}\left(N_{\boldsymbol{\lambda}+\boldsymbol{\rho}}\right)=\sigma_{j}(\boldsymbol{\lambda}) N_{\boldsymbol{\lambda}+\boldsymbol{\rho}}, \quad \Delta_{j}\left(\Phi_{\boldsymbol{\lambda}+\boldsymbol{\rho}}\right)=\sigma_{j}(\boldsymbol{\lambda}) \Phi_{\boldsymbol{\lambda}+\boldsymbol{\rho}}
$$

Corollary 1.2.3 The operators $\left\{\Delta_{j}\right\}_{j=1}^{r}$ are algebraically independent generators of $\mathcal{D}$.

Since $\Omega=\operatorname{GL}(\Omega) / L$ is a Riemannian symmetric space (more precisely, a direct product of $\mathbb{R}_{+}$with an irreducible symmetric space $\Omega^{\prime}:=\{x \in \Omega: N(x)=1\}$ of non-compact type), one has a direct integral decomposition

$$
L^{2}(\Omega)=\int_{\mathbb{R}^{r} / W_{r}} H_{\boldsymbol{\lambda}}|c(\boldsymbol{\lambda})|^{2} d \boldsymbol{\lambda}
$$


where $c(\boldsymbol{\lambda})$ is Harish-Chandra's $c$-function and $H_{\boldsymbol{\lambda}}$ is the Hilbert space completion of the space spanned by all GL( $\Omega$ )-translates of $\Phi_{\boldsymbol{\lambda}}$, endowed with its natural inner product [He84]. Via (1.2.7), the translation representation $T$ of $\mathrm{GL}(\Omega)$ on $L^{2}(\Omega)$ has a decomposition

$$
T=\int_{\mathbb{R}^{r} / W_{r}} T_{\boldsymbol{\lambda}}|c(\boldsymbol{\lambda})|^{-2} d \boldsymbol{\lambda}
$$

where $T_{\boldsymbol{\lambda}}$ is the (irreducible) spherical representation of $\mathrm{GL}(\Omega)$ on $H_{\boldsymbol{\lambda}}$. For any continuous $W_{r}$-invariant function $F: \mathbb{R}^{r} \rightarrow \mathbb{R}$ one can define a $\mathrm{GL}(\Omega)$-invariant self-adjoint operator $\hat{F}$ on $L^{2}(\Omega)$ by the formula

$$
\hat{F} f=\int F(\boldsymbol{\lambda}) f_{\boldsymbol{\lambda}}|c(\boldsymbol{\lambda})|^{-2} d \boldsymbol{\lambda}
$$

for

$$
f=\int f_{\boldsymbol{\lambda}}|c(\boldsymbol{\lambda})|^{-2} d \boldsymbol{\lambda}, \quad f_{\boldsymbol{\lambda}} \in H_{\boldsymbol{\lambda}} .
$$

The domain of $\hat{F}$ is defined as the space of functions $f$ such that

$$
\int|F(\boldsymbol{\lambda})|^{2}\left\|f_{\boldsymbol{\lambda}}\right\|_{\boldsymbol{\lambda}}^{2}|c(\boldsymbol{\lambda})|^{-2} d \boldsymbol{\lambda}<+\infty
$$

Thus $\hat{F}$ is bounded if $F$ is a bounded function. Let

$$
\boldsymbol{\sigma}:=\left(\sigma_{1}, \ldots, \sigma_{r}\right): \mathbb{C}^{r} \rightarrow \mathbb{C}^{r}
$$

where the $\sigma_{j}$ are defined by (1.2.4). The direct integral decomposition above diagonalizes simultaneously the (commuting) operators $\Delta_{k}$. Writing

$$
F=f \circ \sigma
$$

for some continuous bounded function $f: \mathbb{R}^{r} \rightarrow \mathbb{R}$, the bounded operator $\hat{F}$ can be expressed as a function

$$
\hat{F}=f\left(\Delta_{1}, \ldots, \Delta_{r}\right),
$$

in the spectral-theoretic sense, of $\Delta_{1}, \ldots, \Delta_{r}$.

Remark 1.2.4 There are many other natural choices of $r$ algebraically independent generators of $\mathcal{S}$, and each such choice yields $r$ algebraically independent generators of $\mathcal{D}$ via the HarishChandra isomorphism. See [FK94], [N89], [M87], and [M95].

Lemma 1.2.5 Let $U \subset \mathbb{C}^{r}$ be a $W_{r}$-invariant domain, and let $F$ be a $W_{r}$-invariant holomorphic function on $U$.

(i) The associated $G L(\Omega)$-invariant operator $T=\hat{F}$ satisfies

$$
T\left(\Phi_{\boldsymbol{\lambda}+\boldsymbol{\rho}}\right)=F(\boldsymbol{\lambda}) \Phi_{\boldsymbol{\lambda}+\boldsymbol{\rho}} \quad \forall \boldsymbol{\lambda} \in U
$$

(ii) There exists a unique holomorphic function $f$ on $\sigma(U)$ so that $F=f \circ \sigma$, i.e.

$$
F(\boldsymbol{\lambda})=f\left(\sigma_{1}(\boldsymbol{\lambda}), \sigma_{2}(\boldsymbol{\lambda}), \ldots, \sigma_{r}(\boldsymbol{\lambda})\right) \quad \forall \boldsymbol{\lambda} \in U .
$$


(iii) In terms of the $L^{2}$-functional calculus associated with $\left\{\Delta_{j}\right\}_{j=1}^{r}$,

$$
T=f\left(\Delta_{1}, \Delta_{2}, \ldots, \Delta_{r}\right) .
$$

The results described above are valid in the context of the cones $\Omega^{(\ell)}, 1 \leq \ell \leq r$. Thus the ring $\mathcal{S}_{\ell}:=\mathbb{C}\left[\lambda_{1}, \ldots, \lambda_{\ell}\right]^{W_{\ell}}$ of the symmetric polynomials in $\boldsymbol{\lambda}^{(\ell)}:=\left(\lambda_{1}, \ldots, \lambda_{\ell}\right)$ is isomorphic to the full polynomial ring $\mathbb{C}\left[\sigma_{1}, \ldots, \sigma_{\ell}\right]$, and the elementary symmetric polynomials

$$
\sigma_{j}^{(\ell)}\left(\boldsymbol{\lambda}^{(\ell)}\right):=\sum_{1 \leq i_{1}<i_{2}<\cdots<i_{j} \leq \ell} \lambda_{i_{1}} \lambda_{i_{2}} \cdots \lambda_{i_{j}} \quad 1 \leq j \leq \ell
$$

are algebraically independent generators of $\mathcal{S}_{\ell}$. The spherical functions in the context of $\Omega^{(\ell)}$ are parametrized by $\mathbb{C}^{\ell}$ and are defined as before via

$$
\Phi_{\boldsymbol{\lambda}^{(\ell)}}^{(\ell)}(x):=\int_{L^{(\ell)}} N_{\lambda^{(\ell)}}(k(x)) d k, \quad x \in \Omega^{(\ell)} .
$$

The Harish-Chandra isomorphism between $\mathcal{D}_{\ell}=\operatorname{Diff}\left(\Omega^{(\ell)}\right)^{\mathrm{GL}\left(\Omega^{(\ell)}\right)}$ and $\mathcal{S}_{\ell}$ is given via

$$
T\left(\Phi_{\boldsymbol{\lambda}^{(\ell)}+\boldsymbol{\rho}^{(\ell)}}^{(\ell)}\right)=\gamma_{T}^{(\ell)}\left(\boldsymbol{\lambda}^{(\ell)}\right) \Phi_{\boldsymbol{\lambda}^{(\ell)}+\boldsymbol{\rho}^{(\ell)}}^{(\ell)}, \quad \boldsymbol{\lambda}^{(\ell)} \in \mathbb{C}^{(\ell)},
$$

where

$$
\boldsymbol{\rho}^{(\ell)}:=\left(\rho_{1}^{(\ell)}, \rho_{2}^{(\ell)}, \ldots, \rho_{\ell}^{(\ell)}\right), \quad \text { and } \quad \rho_{j}^{(\ell)}:=\frac{a}{4}(2 j-\ell-1) .
$$

The algebraically independent generators of $\mathcal{D}_{\ell}$ are

$$
\Delta_{j}^{(\ell)}:=\left(\gamma^{(\ell)}\right)^{-1}\left(\sigma_{j}^{(\ell)}\right), \quad 1 \leq j \leq \ell .
$$

Lemma 1.2.5 is valid in the context of $\Omega^{(\ell)}$ with obvious notational changes.

Consider the group of linear automorphisms of $\Omega^{(\ell)}$

$$
\mathrm{GL}\left(\Omega^{(\ell)}\right):=\left\{g \in \mathrm{GL}\left(X^{(\ell)}\right) ; g\left(\Omega^{(\ell)}\right)=\Omega^{(\ell)}\right\}
$$

and the associated ring of $\mathrm{GL}\left(\Omega^{(\ell)}\right)$-invariant differential operators

$$
\mathcal{D}_{\ell}:=\operatorname{Diff}\left(\Omega^{(\ell)}\right) \operatorname{GL}\left(\Omega^{(\ell)}\right) .
$$

Thus, $\mathcal{D}_{\ell}$ consists of all differential operators $T$ on $\Omega^{(\ell)}$ so that $T C_{g}=C_{g} T$ for all $g \in \operatorname{GL}\left(\Omega^{(\ell)}\right)$, where $C_{g}(f):=f \circ g$. Let us denote

$$
L^{(\ell)}:=\left\{k \in K ; k\left(u_{\ell}\right)=u_{\ell}\right\} .
$$

Then $k_{\mid \Omega^{(\ell)}} \in \mathrm{GL}\left(\Omega^{(\ell)}\right) \quad \forall k \in L^{(\ell)}$, and in particular $T(f \circ k)=(T f) \circ k$ for all $T \in \mathcal{D}_{\ell}$ and $f \in C^{\infty}\left(\Omega^{(\ell)}\right)$. Let

$$
K^{(\ell)}:=\left\{k \in K ; k\left(Z_{\nu}\left(v_{\ell}\right)\right)=Z_{\nu}\left(v_{\ell}\right), \quad \nu=1, \frac{1}{2}, 0\right\} .
$$

Clearly, $\left\{k \in K ; k\left(v_{\ell}\right)=v_{\ell}\right\} \subset K^{(\ell)}$. Also, every triple-automorphism of $Z_{\nu}\left(v_{\ell}\right)$ for some $\nu=1, \frac{1}{2}, 0$ extends to a triple-automorphism of $Z$ which preserves all the $Z_{\nu}\left(v_{\ell}\right)$, i.e. to an element of $K^{(\ell)}$. Let $K^{(\ell)}$ Cenote the complexification of $K^{(\ell)}$. We need the following technical result. 
Lemma 1.2.6 Every $T \in \mathcal{D}_{\ell}$ extends uniquely to a holomorphic differential operator on $Z_{0}\left(v_{\ell}\right)$ which is invariant under the group $K^{(\ell)}{ }^{\mathbb{C}}$.

Replacing $Z_{0}\left(v_{\ell}\right)$ by $Z$ it is enough to show

Lemma 1.2.7 Let $Z=Z_{1}(e) \oplus Z_{1 / 2}(e)$ be the Peirce decomposition of $Z$ with respect to the maximal tripotent e (cf. (1.1.6)). Then every $G L(\Omega)$-invariant differential operator $D$ on $\Omega$ has a unique extension to a holomorphic differential operator $\tilde{D}$ on $Z$ which is $K^{\mathbb{C}}$-invariant.

Proof: It is well-known that the ring $\operatorname{Diff}(\Omega)^{\mathrm{GL}(\Omega)}$ of all invariant differential operators on $\Omega$ is a (commutative) polynomial algebra in $r$ algebraically independent generators $D_{1}, \ldots, D_{r}$. According to $[\mathrm{N} 89$, p. 130$]$ we may assume

$$
D_{j}=p_{j}\left(x, \frac{\partial}{\partial x}\right) \quad(1 \leq j \leq r)
$$

and

$$
\begin{aligned}
p_{2 j-1}(x, y) & =\left(\left(P_{x} P_{y}\right)^{j-1} x \mid y\right) \\
p_{2 j}(x, y) & =\left(\left(P_{x} P_{y}\right)^{j-1} x \mid P_{y} x\right)
\end{aligned}
$$

where $P_{x}$ is the quadratic representation of $X:=\left\{x \in Z_{1}(e): x^{*}=x\right\}$. Let $\left\{u v^{*} w\right\}$ denote the Jordan triple product of $Z$ and put

$$
Q_{Z} w:=\left\{z w^{*} z\right\}
$$

Define

$$
\begin{aligned}
q_{2 j-1}(z, w) & :=\left(\left(Q_{Z} Q_{w}\right)^{j-1} z \mid w\right) \\
q_{2 j}(z, w) & :=\left(\left(Q_{Z} Q_{w}\right)^{j-1} z \mid Q_{w} z\right)
\end{aligned}
$$

for $z, w \in Z$. Then we have

$$
q_{m}(x, y)=p_{m}(x, y)
$$

for all $x, y \in X$.

By [U85a, p. 297, (18.2.1)], we have

$$
\left\{a u^{*}\left\{a u^{*} a\right\}\right\}=\left\{a\left\{u a^{*} v\right\}^{*} a\right\} .
$$

We use this identity to show that

$$
Q_{z} Q_{w}^{j} z=\left(z \square w^{*}\right)^{2 j} z
$$

for all $j \geq 1$. In fact

$$
Q_{z} Q_{w} z=\left\{z\left\{w z^{*} w\right\}^{*} z\right\}=\left\{z w^{*}\left\{z w^{*} z\right\}\right\}=\left(z \square w^{*}\right)^{2} z .
$$

Now let $j \geq 1$ and put

$$
V:=\left(z \square w^{*}\right)^{2 j-2}=\left(Q_{z} Q_{w}\right)^{j-1} z
$$


and

$$
u:=\left(z \square x^{*}\right)^{2 j-1} z=\left\{z w^{*} v\right\}
$$

Then we have by induction

$$
\begin{aligned}
& Q_{z} Q_{w} v=\left(Q_{z} Q_{w}\right)^{j} z=\left(z \square w^{*}\right)^{2 j} z \\
& Q_{w} u=\left\{w\left\{z w^{*} v\right\}^{*}\right\}=\left\{w z^{*}\left\{w v^{*} w\right\}\right\}=\left\{w z^{*}\left(Q_{w} v\right)\right\} \\
& Q_{z} Q_{w} u=\left\{z\left\{z\left\{w w^{+}\left(Q_{w} v\right)\right\}^{*} z\right\}=\right. \\
& \quad\left\{z w^{*}\left\{z\left(Q_{w} v\right)^{*} z\right\}\right\}=\left\{z w^{*}\left(Q_{z} Q_{w} v\right)\right\}=\left(z \square w^{*}\right)^{2 j+1} z
\end{aligned}
$$

and hence

$$
\begin{aligned}
& \left(Q_{z} Q_{w}\right)^{j+1} z=Q_{z} Q_{w}\left(\left(z \square w^{*}\right)^{2 j} z\right)=Q_{z} Q_{w}\left\{z w^{*} u\right\} \\
& \quad=\left\{z\left\{w\left\{z w^{+} u\right\}^{*} w\right\}^{*} z\right\}=\left\{z\left\{w z^{*}\left\{w u^{*} w\right\}\right\}^{*} z\right\} \\
& \quad=\left\{z w^{*}\left\{z\left\{w u^{*} w\right\}^{*} z\right\}\right\}=\left\{z w^{*}\left(Q_{z} Q_{w} u\right)\right\} \\
& \quad=\left(z \square w^{*}\right)^{2 j+2} z .
\end{aligned}
$$

This completes the induction and proves (1. ).

Combining (1. ) and (1. ), we see that

$$
q_{m}(z, w)=\left(\left(z \square w^{*}\right)^{m-1} z \mid w\right)
$$

for all $m \geq 1$. In fact, (1. ) implies

$$
q_{2 j-1}(z, w)=\left(\left(Q_{z} Q_{w}\right)^{j-1} z \mid w\right)=\left(\left(z \square w^{*}\right)^{2 j-2} z \mid w\right)
$$

and

$$
\begin{aligned}
& q_{2 j}(z, w)=\left(\left(Q_{z} Q_{w}\right)^{j-1} z \mid Q_{w} z\right)= \\
& \left(\left(z \square w^{*}\right)^{2 j-2} z \mid\left\{w z^{*} w\right\}\right)=\left(\left\{\left(\left(z \square w^{*}\right)^{2 j-2} z\right) w^{*} z\right\} \mid w\right) \\
& =\left(\left(z \square w^{*}\right)^{2 j-1} z \mid w\right)
\end{aligned}
$$

since $\left(\left\{u v^{*} w\right\} \mid z\right)=\left(u \mid\left\{v w^{*} z\right\}\right)$ for all $u, v, w, z \in Z$. Now let $b_{1}, \ldots, b_{k} \in X$ and $b_{k+1}, \ldots, b_{n} \in Z_{1 / 2}(e)$ be orthonormal basis. Then

$$
w=\sum_{i=1}^{n}\left(w \mid b_{i}\right) b_{i}
$$

and hence

$$
\begin{gathered}
q_{m}(z, w)=\sum_{1 \leq i_{m} \leq n}\left(\left(\sum_{1 \leq i \leq n}\left(b_{i} \mid w\right) z \square b_{i}^{*}\right)^{m-1} z \mid b_{i m}\right)\left(b_{i m} \mid w\right) \\
=\sum_{1 \leq i_{1}, \ldots, i_{m} \leq n}\left(\left(z \square b_{i_{1}}^{*}\right) \cdots\left(z \square b_{i_{m-1}}^{*}\right) z \mid b_{i m}\right)\left(b_{i_{1}} \mid w\right) \cdots\left(b_{i m} \mid w\right) .
\end{gathered}
$$

It follows that

$$
q_{m}\left(z, \frac{\partial}{\partial z}\right)=\sum_{1 \leq i_{1}, \ldots, i_{m} \leq n}\left(\left(z \square b_{i_{1}}^{*}\right) \cdots\left(z \square b_{i_{m-1}}^{*}\right) z \mid b_{i m}\right) \frac{\partial}{\partial z_{i_{1}}} \cdots \frac{\partial}{\partial z_{i_{m}}} .
$$


For $x \in X$ we have

$$
\left\{x b_{i}^{*} x\right\} \in X \quad(1 \leq i \leq k)
$$

and

$$
\left\{x b_{i}^{*} x\right\}=0 \quad(k<i \leq n)
$$

by the Peirce multiplication rules. It follows that

$$
\left(\left(x \square b_{i_{1}}^{*}\right) \cdots\left(x \square b_{i_{m-1}}^{*}\right) x \mid b_{i_{m}}\right)=0
$$

unless $i_{1}, \ldots, i_{m} \leq k$. This shows that the holomorphic differential operator

$$
\tilde{D}_{m}:=q_{m}\left(z, \frac{\partial}{\partial z}\right)
$$

agrees with

$$
D_{m}=p_{m}\left(x, \frac{\partial}{\partial x}\right)
$$

when restricted to $X$. Since $q_{m}(k z, k w)=q_{m}(z, w)$ for all $k \in K, \tilde{D}_{m}$ is $K$-invariant and hence, by holomorphicity, even $K^{\mathbb{C}}$-invariant.

\subsection{Conialization of functions}

In this section we study conialization (i.e. "conical polarization") of functions on $Z$. The basic fact used here is that every $z \in Z$ admits a conical polar decomposition $z=k(x)$ with $k \in K$ and a unique $x \in \Omega$. Thus $Z=K \cdot \Omega$, and we have a formula for integration in conical polar coordinates for functions $f \in L^{1}(Z, m)$ :

$$
\int_{Z} f(z) d m(z)=c_{0} \int_{\Omega}\left(\int_{K} f\left(k\left(x^{\frac{1}{2}}\right)\right) d k\right) N(x)^{b} d x
$$

where $m$ is Lebesgue measure, and $c_{0}=\pi^{d} / \Gamma_{\Omega}\left(\frac{d}{r}\right)$. The function

$$
\tilde{f}(x):=\int_{K} f\left(k\left(x^{\frac{1}{2}}\right)\right) d k, \quad x \in \Omega,
$$

is called the conialization of $f$. The map $E(f)(x):=\tilde{f}\left(x^{2}\right)$ can be considered as the averaging projection (i.e. conditional expectation) from $L^{1}(Z, m)$ onto its subspace of $K$-invariant functions.

Lemma 1.3.1 (i) For every partition $\mathbf{m}$ and every $x \in \Omega$

$$
\int_{K}\left|\Phi_{\mathbf{m}}\left(k\left(x^{\frac{1}{2}}\right)\right)\right|^{2} d k=\frac{\Phi_{\mathbf{m}}(x)}{d_{\mathbf{m}}},
$$

where $d_{\mathbf{m}}:=\operatorname{dim}\left(P_{\mathbf{m}}\right)$.

(ii) For every $x \in \Omega$ and all polynomials $f=\sum_{\mathbf{m}} f_{\mathbf{m}}$ and $g=\sum_{\mathbf{m}} g_{\mathbf{m}}$ with $f_{\mathbf{m}}, g_{\mathbf{m}} \in P_{\mathbf{m}}$ for all $\mathbf{m}$,

$$
\widetilde{(f \bar{g})}(x)=\sum_{\mathbf{m}}<f_{\mathbf{m}}, g_{\mathbf{m}}>_{\frac{d}{r}} \Phi_{\mathbf{m}}(x)
$$


Proof: Formula (1.3.3) is proved in [FK94], Proposition XI.4.1 in the case where $Z$ is a JB*algebra, and in [FK90] in the case where $Z$ is a $\mathrm{JB}^{*}$-triple. Notice that (1.3.3) with $x=e$ yields for every $\mathbf{m}$

$$
\left\|\Phi_{\mathbf{m}}\right\|_{\frac{d}{r}}^{2}=\int_{K}\left|\Phi_{\mathbf{m}}(k(e))\right|^{2} d k=\frac{1}{d_{\mathbf{m}}} .
$$

To prove (1.3.4), consider the $K$-invariant inner product

$$
<f, g>_{x}:=\widetilde{(f \bar{g})}(x)=\int_{K} f\left(k\left(x^{\frac{1}{2}}\right)\right) \overline{g\left(k\left(x^{\frac{1}{2}}\right)\right)} d k
$$

on $\mathcal{P}$. Using the fact that the actions of $K$ on the $P_{\mathbf{m}}$ are irreducible and pair-wise inequivalent, we see that the $P_{\mathbf{m}}$ are pair-wise orthogonal with respect to $\langle\cdot, \cdot\rangle_{x}$, and that there exist positive constants $c_{\mathbf{m}}(x)$ so that

$$
<f_{\mathbf{m}}, g_{\mathbf{m}}>_{x}=c_{\mathbf{m}}(x)<f_{\mathbf{m}}, g_{\mathbf{m}}>_{\frac{d}{r}}, \quad \forall f_{\mathbf{m}}, g_{\mathbf{m}} \in P_{\mathbf{m}} .
$$

The proportionality constants are computed by taking $f_{\mathbf{m}}=g_{\mathbf{m}}=\Phi_{\mathbf{m}}$ and using (1.3.3) for $x$ and $e$.

Let $1 \leq \ell \leq r$ and denote the vectors in $\mathbb{C}^{\ell}$ by $\boldsymbol{\lambda}^{(\ell)}=\left(\lambda_{1}, \ldots, \lambda_{\ell}\right)$. For notational simplicity we shall adopt the convention that $\Phi_{\boldsymbol{\lambda}^{(\ell)}}=\Phi_{\left(\lambda_{1}, \ldots, \lambda_{\ell}, 0, \ldots, 0\right)}$, and similarly for the conical functions. Recall that the spherical functions associated with the symmetric cone $\Omega^{(\ell)}$ of $X^{(\ell)}$ are denoted by $\Phi_{\lambda^{(\ell)}}^{(\ell)}$.

Proposition 1.3.2 Let $1 \leq \ell \leq r$ and let $\mathbf{m}^{(\ell)}=\left(m_{1}, \ldots, m_{\ell}\right) \in \mathbb{N}^{\ell}$ be a partition. Then for every $x \in X^{(\ell)}$

$$
\Phi_{\mathbf{m}^{(\ell)}}(x)=\gamma_{\mathbf{m}^{(\ell)}} \Phi_{\mathbf{m}^{(\ell)}}^{(\ell)}(x),
$$

where

$$
\gamma_{\mathbf{m}^{(\ell)}}=\frac{\left(\ell \frac{a}{2}\right)_{\mathbf{m}^{(\ell)}}}{\left(r \frac{a}{2}\right)_{\mathbf{m}^{(\ell)}}}=\frac{\Gamma_{\Omega^{(\ell)}}\left(r \frac{a}{2}\right)}{\Gamma_{\Omega^{(\ell)}}\left(\ell \frac{a}{2}\right)} \prod_{j=1}^{\ell} \frac{\Gamma\left(m_{j}+(\ell+1-j) \frac{a}{2}\right)}{\Gamma\left(m_{j}+(r+1-j) \frac{a}{2}\right)} .
$$

Proof: Recall that for every $y \in X$ and $\lambda \in \mathbb{C}$,

$$
N(e-y)^{-\lambda}=\sum_{\mathbf{m}}(\lambda)_{\mathbf{m}} \frac{\Phi_{\mathbf{m}}(y)}{\left\|\Phi_{\mathbf{m}}\right\|_{F}^{2}} .
$$

Similarly, for $x \in X^{(\ell)}$ and $\lambda \in \mathbb{C}$,

$$
N(e-x)^{-\lambda}=N_{\ell}\left(u_{\ell}-x\right)^{-\lambda} \sum_{\mathbf{m}^{(\ell)}}(\lambda)_{\mathbf{m}^{(\ell)}} \frac{\Phi_{\mathbf{m}^{(\ell)}}^{(\ell)}(x)}{\left\|\Phi_{\mathbf{m}^{(\ell)}}^{(\ell)}\right\|_{F}^{2}} .
$$

In order to continue the proof of the proposition, we need the following result.

Lemma 1.3.3 Let $1 \leq \ell \leq r$ and let $y \in X$ be an element of rank at most $\ell$. If $\mathbf{n}=\left(n_{1}, \ldots, n_{r}\right)$ is a partition with $n_{\ell+1} \geq 1$, then $N_{\mathbf{n}}(y)=\Phi_{\mathbf{n}}(y)=0$. 
Proof: The condition $n_{\ell+1} \geq 1$ guarantees that for some $j>\ell, N_{\mathbf{n}}$ is divisible by $N_{j}$ to a positive power. Notice that $\operatorname{rank}\left(P_{1}\left(u_{j}\right) y\right) \leq \operatorname{rank}(y) \leq \ell$. Hence, $N_{j}(y)=N_{j}\left(P_{1}\left(u_{j}\right) y\right)=0$ (because in the Jordan algebra $X^{(j)}$ elements of rank smaller than $j$ have zero determinant). In particular, $N_{\mathbf{n}}(y)=0$. If $k \in L$ then $\operatorname{rank}(k(y))=\operatorname{rank}(y) \leq \ell$, and therefore $N_{\mathbf{n}}(k(y))=0$. Finally, $\Phi_{\mathbf{n}}(y)=\int_{L} N_{\mathbf{n}}(k(y)) d k=0$.

Using Lemma 1.3.3 we see that (1.3.8) for $x \in X^{(\ell)}$ yields

$$
N(e-x)^{-\lambda}=\sum_{\mathbf{m}^{(\ell)}}(\lambda)_{\mathbf{m}^{(\ell)}} \frac{\Phi_{\mathbf{m}^{(\ell)}}(x)}{\left\|\Phi_{\mathbf{m}^{(\ell)}}\right\|_{F}^{2}} .
$$

Since $\Phi_{\mathbf{m}^{(\ell)} \mid Z_{0}\left(v_{\ell}\right)} \in P_{\mathbf{m}}^{(\ell)}$, we obtain by comparing the expansions (1.3.9) and (1.3.10) that

$$
\Phi_{\mathbf{m}^{(\ell)}}(x)=\frac{\left\|\Phi_{\mathbf{m}^{(\ell)}}\right\|_{F}^{2}}{\left\|\Phi_{\mathbf{m}^{(\ell)}}^{(\ell)}\right\|_{F}^{2}} \Phi_{\mathbf{m}^{(\ell)}}^{(\ell)}(x)=\gamma_{\mathbf{m}^{(\ell)}} \Phi_{\mathbf{m}^{(\ell)}}^{(\ell)}(x) \quad \forall x \in X^{(\ell)} .
$$

In order to compute $\gamma_{\mathbf{m}^{(\ell)}}$ we use the known fact (see [FK90]) that

$$
\left\|\Phi_{\mathbf{m}^{(\ell)}}\right\|_{F}^{2}=\frac{\left(\frac{d}{r}\right)_{\mathbf{m}^{(\ell)}}}{d_{\mathbf{m}^{(\ell)}}} \text { and }\left\|\Phi_{\mathbf{m}^{(\ell)}}^{(\ell)}\right\|_{F}^{2}=\frac{\left(\frac{d_{\ell}}{\ell}\right)_{\mathbf{m}^{(\ell)}}}{d_{\mathbf{m}^{(\ell)}}^{(\ell)}},
$$

where $d_{\ell}:=\operatorname{dim} Z_{1}\left(u_{\ell}\right)=\ell+\ell(\ell-1) \frac{a}{2}, d_{\mathbf{m}^{(\ell)}}=\operatorname{dim}\left(P_{\mathbf{m}^{(\ell)}}\right)$, and $d_{\mathbf{m}^{(\ell)}}^{(\ell)}$ has the same meaning with respect to the algebra $Z_{1}\left(u_{\ell}\right)$. Quite generally, the dimensions $d_{\mathbf{m}}$ are expressed by

$$
d_{\mathbf{m}}=\prod_{1 \leq i<j \leq r} \frac{B\left((j-i) \frac{a}{2}, \frac{a}{2}\right)}{B\left(m_{i}-m_{j}+(j-i) \frac{a}{2}, \frac{a}{2}\right)} \frac{B\left((i-j) \frac{a}{2}, \frac{a}{2}\right)}{B\left(m_{j}-m_{i}+(i-j) \frac{a}{2}, \frac{a}{2}\right)}
$$

where $B(x, y):=\Gamma(x) \Gamma(y) / \Gamma(x+y)$ is the ordinary Beta function. (see [U83] for the general case, and [FK94], p. 315 for the case of $\mathrm{JB}^{*}$-algebras).

A straightforward computation yields the expression (1.3.7) for $\gamma_{\mathbf{m}^{(\ell)}}$.

Remark 1.3.4 One can prove Proposition 1.3.2 using the connection between the spherical polynomials and the Jack symmetric functions $J_{\mathbf{m}}^{(\alpha)}$, where $\alpha:=\frac{2}{a}$, and $\mathbf{m}$ ranges over all finite partitions. (See [M87], [M95] and [St89] for the study of Jack symmetric functions). $J_{\boldsymbol{\lambda}}^{(\alpha)}$ is defined on all finite sequences (identified with infinite sequences which contain only finitely many non-zero terms), and it is permutation invariant. The connecting formula is

$$
\Phi_{\mathbf{m}}\left(\sum_{j=1}^{r} t_{j} e_{j}\right)=\frac{J_{\mathbf{m}}^{(\alpha)}\left(t_{1}, \ldots, t_{r}, 0, \ldots, 0, \ldots\right)}{J_{\mathbf{m}}^{(\alpha)}\left(1^{r}\right)} \quad \forall t_{1}, \ldots, t_{r}>0,
$$

where $1^{r}:=(1, \ldots, 1,0, \ldots, 0, \ldots)$ has $r$ "1". A similar formula is valid also for the spherical functions $\Phi_{\mathbf{m}^{(\ell)}}^{(\ell)}$ associated with $\Omega^{(\ell)}$ :

$$
\Phi_{\mathbf{m}^{(\ell)}}^{(\ell)}\left(\sum_{j=1}^{\ell} t_{j} e_{j}\right)=\frac{J_{\mathbf{m}^{(\ell)}}^{(\alpha)}\left(t_{1}, \ldots, t_{\ell}, 0, \ldots, 0, \ldots\right)}{J_{\mathbf{m}^{(\ell)}}^{(\alpha)}\left(1^{\ell}\right)} \quad \forall t_{1}, \ldots, t_{\ell}>0,
$$


It follows that for every $t_{1}, \ldots, t_{\ell}>0$,

$$
\frac{\Phi_{\mathbf{m}^{(\ell)}}\left(\sum_{j=1}^{\ell} t_{j} e_{j}\right)}{\Phi_{\mathbf{m}^{(\ell)}}^{(\ell)}\left(\sum_{j=1}^{\ell} t_{j} e_{j}\right)}=\frac{J_{\mathbf{m}^{(\ell)}}^{(\alpha)}\left(1^{\ell}\right)}{J_{\mathbf{m}^{(\ell)}}^{(\alpha)}\left(1^{r}\right)}=\gamma_{\mathbf{m}^{(\ell)}} .
$$

The numbers $J_{\mathbf{m}}^{(\alpha)}\left(1^{\nu}\right), \nu \in \mathbb{N}$, are known in full generality (see [St89] Th. 5.4 and [M95]):

$$
J_{\mathbf{m}}^{(\alpha)}\left(1^{\nu}\right)=\prod_{i=1}^{\ell(\mathbf{m})} \prod_{j=1}^{m_{i}}(\nu+1-i+\alpha(j-1))
$$

where $\ell(\mathbf{m}):=\max \left\{k ; m_{k} \neq 0\right\}$ is the length of $\mathbf{m}$. It follows that if $\ell(\mathbf{m}) \leq r$ and $\alpha=\frac{2}{a}$, then

$$
J_{\mathbf{m}}^{(\alpha)}\left(1^{\nu}\right)=\left(\frac{2}{a}\right)^{|\mathbf{m}|} \prod_{j=1}^{r} \frac{\Gamma\left(m_{j}+(\nu+1-j) \frac{a}{2}\right)}{\Gamma\left((\nu+1-j) \frac{a}{2}\right)}=\left(\frac{2}{a}\right)^{|\mathbf{m}|}\left(\nu \frac{a}{2}\right)_{\mathbf{m}} .
$$

In particular,

$$
\gamma_{\mathbf{m}^{(\ell)}}=\frac{J_{\mathbf{m}^{(\ell)}}^{(\alpha)}\left(1^{\ell}\right)}{J_{\mathbf{m}^{(\ell)}}^{(\alpha)}\left(1^{r}\right)}=\frac{\left(\ell \frac{a}{2}\right)_{\mathbf{m}^{(\ell)}}}{\left(r \frac{a}{2}\right)_{\mathbf{m}^{(\ell)}}}=\frac{\Gamma_{\Omega^{(\ell)}}\left(r \frac{a}{2}\right)}{\Gamma_{\Omega^{(\ell)}}\left(\ell \frac{a}{2}\right)} \prod_{j=1}^{\ell} \frac{\Gamma\left(m_{j}+(\ell+1-j) \frac{a}{2}\right)}{\Gamma\left(m_{j}+(r+1-j) \frac{a}{2}\right)},
$$

where $\Gamma_{\Omega^{(\ell)}}$ is the Gamma function associated with the cone $\Omega^{(\ell)}$. The spectral theorem in $X$ [Lo77] and the fact that $L$ acts transitively on the frames of primitive idempotents in $X$ imply that every spherical polynomial $\Phi_{\mathbf{m}}$ is determined by its restriction to $\operatorname{span}\left\{e_{j}\right\}_{j=1}^{r}$. Thus (1.3.6) in general follows from (1.3.6) for $x=\sum_{j=1}^{\ell} t_{j} e_{j}$, i.e. from (1.3.14).

Remark 1.3.5 Recall that the half sum of the strongly orthogonal positive roots associated with $\Omega^{(\ell)}$ is $\boldsymbol{\rho}^{(\ell)}:=\left(\rho_{1}^{(\ell)}, \ldots \rho_{\ell}^{(\ell)}\right), \quad \rho_{j}^{(\ell)}:=(2 j-\ell-1) \frac{a}{4}$. For any partition $\mathbf{m}^{(\ell)}=\left(m_{1}, \ldots, m_{\ell}\right)$ define $\boldsymbol{\lambda}^{(\ell)}=\left(\lambda_{1}, \ldots, \lambda_{\ell}\right)$ via the " $\rho^{(\ell)}$-shift"

$$
\boldsymbol{\lambda}^{(\ell)}:=\mathbf{m}^{(\ell)}-\boldsymbol{\rho}^{(\ell)} \text {, namely } \lambda_{j}:=m_{j}-\rho_{j}^{(\ell)}=m_{j}-(2 j-\ell-1) \frac{a}{4}, \quad 1 \leq j \leq \ell .
$$

Then $\gamma_{\mathbf{m}^{(\ell)}}$ can be written as a symmetric function of $\boldsymbol{\lambda}^{(\ell)}=\left(\lambda_{1}, \ldots, \lambda_{\ell}\right)$ :

$$
\gamma_{\mathbf{m}^{(\ell)}}=\frac{\Gamma_{\Omega^{(\ell)}}\left(r \frac{a}{2}\right)}{\Gamma_{\Omega^{(\ell)}}\left(\ell \frac{a}{2}\right)} \prod_{j=1}^{\ell} \frac{\Gamma\left(\lambda_{j}+(\ell+1) \frac{a}{4}\right)}{\Gamma\left(\lambda_{j}+(2 r-\ell+1) \frac{a}{4}\right)} .
$$

This will be crucial in the sequel.

Recall that $\mathcal{H}_{\ell \frac{a}{2}}$ is the completion of $\mathcal{P}_{\ell}$ (see (1.1.18)) with respect to the inner product (1.1.20) with $\lambda=\ell \frac{a}{2}$.

Corollary 1.3.6 For all functions $f, g \in \mathcal{H}_{\ell \frac{a}{2}}$ with expansions $f=\sum_{\mathbf{m}^{(\ell)}} f_{\mathbf{m}^{(\ell)}}$ and $g=$ $\sum_{\mathbf{m}^{(\ell)}} g_{\mathbf{m}^{(\ell)}}$, and for every $x \in \Omega^{(\ell)}$,

$$
\begin{aligned}
\widetilde{(f \bar{g})}(x) & =\sum_{\mathbf{m}^{(\ell)}}<f_{\mathbf{m}^{(\ell)}}, g_{\mathbf{m}^{(\ell)}}>_{\frac{d}{r}} \frac{\left(\ell \frac{a}{2}\right)_{\mathbf{m}^{(\ell)}}}{\left(r \frac{a}{2}\right)_{\mathbf{m}^{(\ell)}}} \Phi_{\mathbf{m}^{(\ell)}}^{(\ell)}(x) \\
& =\frac{\Gamma_{\Omega^{(\ell)}}\left(r \frac{a}{2}\right)}{\Gamma_{\Omega^{(\ell)}}\left(\ell \frac{a}{2}\right)} \sum_{\mathbf{m}^{(\ell)}}\left(\prod_{j=1}^{\ell} \frac{\Gamma\left(\lambda_{j}+(\ell+1) \frac{a}{4}\right)}{\Gamma\left(\lambda_{j}+(2 r-\ell+1) \frac{a}{4}\right)} \Phi_{\mathbf{m}^{(\ell)}}^{(\ell)}(x)\right)<f_{\mathbf{m}^{(\ell)}}, g_{\mathbf{m}^{(\ell)}}>_{\frac{d}{r}},
\end{aligned}
$$


where, as before,

$$
\lambda_{j}=m_{j}-\rho_{j}^{(\ell)}=m_{j}-(2 j-\ell-1) \frac{a}{4}, \quad 1 \leq j \leq \ell .
$$

The point is that the coefficients of $\left\langle f_{\mathbf{m}^{(\ell)}}, g_{\mathbf{m}^{(\ell)}}\right\rangle_{\frac{d}{r}}$ in the expansion of $\widetilde{(f \bar{g})}(x)$ are symmetric functions of $\boldsymbol{\lambda}^{\ell}=\left(\lambda_{1}, \ldots, \lambda_{\ell}\right)$.

\subsection{Integration on $K$-orbits}

In this section we will be interested in two sequences of $K$-orbits. The first sequence is the $G$-orbits $\left\{\partial_{j} D\right\}_{j=1}^{r}$ on $\partial D$. Notice that $\partial_{r-\ell} D=K\left(B\left(v_{\ell}\right)\right)$, where $v_{\ell}=e_{\ell+1}+\cdots+e_{r}$. Recall that $u_{\ell}=e_{1}+\cdots+e_{\ell}$, and denote the open unit interval in the cone $\Omega^{(\ell)}$ by

$$
I^{(\ell)}:=\Omega^{(\ell)} \cap\left(u_{\ell}-\Omega^{(\ell)}\right)=\left\{x \in X^{(\ell)} ; 0<x<u_{\ell}\right\} .
$$

The second sequence of $K$-orbits that we shall need is

$$
\mathcal{O}_{\ell}:=K\left(I^{(\ell)}\right), \quad 1 \leq \ell \leq r .
$$

Note that $\partial_{r-\ell} D=K\left(v_{\ell}+I^{(\ell)}\right)$ and $\mathcal{O}_{\ell}$ are the $K$-orbits of the opposite faces $I^{(\ell)}$ and $v_{\ell}+I^{(\ell)}$ of the unit interval $I:=\Omega \cap(e-\Omega)$ of the cone $\Omega$.

We shall use the subgroup

$$
G_{v_{\ell}}:=\left\{\varphi \in G ; \varphi\left(v_{\ell}\right)=v_{\ell}\right\}
$$

of $G$, identified naturally with $\operatorname{Aut}\left(D_{0}\left(v_{\ell}\right)\right)$, and the subgroups $K_{v_{\ell}}:=K \cap G_{v_{\ell}}$ and

$$
K^{(\ell)}:=\left\{k \in K ; k\left(Z_{\nu}\left(v_{\ell}\right)\right)=Z_{\nu}\left(v_{\ell}\right), \quad \nu=1, \frac{1}{2}, 0\right\}
$$

of $K$.

We describe now a construction which assigns to a measure $\nu$ on $\overline{I^{(\ell)}}$ measures $\hat{\mu}$ and $\tilde{\mu}$ on the orbits $\partial_{r-\ell} D$ and $\mathcal{O}_{\ell}$ respectively. The construction uses as an intermediate step a construction of a measure $\mu$ on $\overline{D_{0}\left(v_{\ell}\right)}$.

Let $\nu$ be a measure on $\overline{I^{(\ell)}}$, and define a measure $\mu$ (depending on $\nu$ ) on $\overline{D_{0}\left(v_{\ell}\right)}$ via

$$
\int_{\overline{D_{0}\left(v_{\ell}\right)}} f d \mu=\int_{\overline{I^{(\ell)}}}\left(\int_{K_{\nu_{\ell}}} f\left(k\left(x^{\frac{1}{2}}\right)\right) d k\right) d \nu(x) .
$$

We call $\nu$ the conical part of $\mu$. Using $\mu$ we construct measures $\tilde{\mu}$ and $\hat{\mu}$ on the $K$-orbits $\mathcal{O}_{\ell}$ and $\partial_{r-\ell} D$ in a canonical way.

Construction of $\tilde{\mu}$ : We define

$$
\begin{aligned}
\int_{\mathcal{O}_{\ell}} f d \tilde{\mu} & :=\int_{\overline{D_{0}\left(v_{\ell}\right)}}\left(\int_{K} f(k(z)) d k\right) d \mu(z) \\
& =\int_{\overline{I^{(\ell)}}}\left(\int_{K} f\left(k\left(x^{\frac{1}{2}}\right)\right) d k\right) d \nu(x)=\int_{\overline{I^{(\ell)}}} \tilde{f}(x) d \nu(x) .
\end{aligned}
$$


Example 1.4.1 Let $\lambda>p_{\ell}-1$ (where $p_{\ell}:=(\ell-1) a+2+b$ is the genus of $D_{0}\left(v_{\ell}\right)$ ), and consider the probability measure

$$
d \mu_{\lambda}^{(\ell)}(z):=c^{(\ell)} h_{\ell}(z, z)^{\lambda-p_{\ell}} d m(z), \quad c^{(\ell)}=\frac{\Gamma_{\Omega^{(\ell)}}(\lambda)}{\pi^{\ell} \Gamma_{\Omega^{(\ell)}}\left(\lambda-\frac{d^{(\ell)}}{\ell}\right)}
$$

on $D_{0}\left(v_{\ell}\right)$, where $d^{(\ell)}:=\operatorname{dim} Z_{0}\left(v_{\ell}\right)=\ell(\ell-1) \frac{a}{2}+\ell+\ell b$, and

$$
h_{\ell}\left(k\left(x^{\frac{1}{2}}\right), k\left(x^{\frac{1}{2}}\right)\right)=N_{\ell}\left(u_{\ell}-x\right), \quad \forall x \in I^{(\ell)}, \forall k \in K^{(\ell)} .
$$

The conical part of $d \mu_{\lambda}^{(\ell)}$ is the probability measure

$$
d \nu_{\lambda}^{(\ell)}(x):=\frac{1}{\mathrm{~B}_{\Omega^{(\ell)}}\left(\frac{d^{(\ell)}}{\ell}, \lambda-\frac{d^{(\ell)}}{\ell}\right)} N_{\ell}\left(u_{\ell}-x\right)^{\lambda-p_{\ell}} N_{\ell}(x)^{b} d m(x)
$$

on $I^{(\ell)}$, where $\mathrm{B}_{\Omega^{(\ell)}}$ is the Beta function associated with the cone $\Omega^{(\ell)}$ (see (1.1.14)).

Example 1.4.2 For $\lambda=\frac{d^{(\ell)}}{\ell}$ we consider the probability measure $\sigma_{\ell}$ on the Shilov boundary $\partial_{\ell} D_{0}\left(v_{\ell}\right)$ of $D_{0}\left(v_{\ell}\right)$ :

$$
\int_{\partial_{\ell} D_{0}\left(v_{\ell}\right)} f d \sigma_{\ell}:=\int_{K^{(\ell)}} f\left(k\left(u_{\ell}\right)\right) d k .
$$

Its conical part is the Dirac measure $\delta_{u_{\ell}}$.

Note that with respect to the measures $\mu_{\lambda}^{(\ell)}$ and $\sigma_{\ell}$ considered in Examples 1.4.1 and 1.4.2, we have

$$
\left\|\Phi_{\mathbf{m}^{(\ell)}}^{(\ell)}\right\|_{L^{2}\left(\mu_{\lambda}^{(\ell)}\right)}^{2}=\frac{1}{d_{\mathbf{m}^{(\ell)}}^{(\ell)}} \int_{I^{(\ell)}} \Phi_{\mathbf{m}^{(\ell)}}^{(\ell)}(x) d \nu_{\lambda}^{(\ell)}(x)=\frac{\left\|\Phi_{\mathbf{m}^{(\ell)}}^{(\ell)}\right\|_{F}^{2}}{(\lambda)_{\mathbf{m}^{(\ell)}}}
$$

and

$$
\left\|\Phi_{\mathbf{m}^{(\ell)}}^{(\ell)}\right\|_{L^{2}\left(\sigma_{\ell}\right)}^{2}=\frac{\left\|\Phi_{\mathbf{m}^{(\ell)}}^{(\ell)}\right\|_{F}^{2}}{\left(\frac{d^{(\ell)}}{\ell}\right)_{\mathbf{m}^{(\ell)}}}=\frac{1}{\left(\frac{d^{(\ell)}}{\ell}\right)_{\mathbf{m}^{(\ell)}}} .
$$

Applying Corollary 1.3.6, and using (1.4.8) and (1.4.9), we obtain

Corollary 1.4.3 Let $f, g \in \mathcal{P}_{\ell}$ have expansions $f=\sum_{\mathbf{m}^{(\ell)}} f_{\mathbf{m}^{(\ell)}}$ and $g=\sum_{\mathbf{m}^{(\ell)}} g_{\mathbf{m}^{(\ell)}}$. Then

(i)

$$
<f, g>_{L^{2}(\mathcal{O}, \tilde{\mu})}=\sum_{\mathbf{m}^{(\ell)}} \frac{\left(\ell \frac{a}{2}\right)_{\mathbf{m}^{(\ell)}}}{\left(r \frac{a}{2}\right)_{\mathbf{m}^{(\ell)}}} \int_{\overline{I^{(\ell)}}} \Phi_{\mathbf{m}^{(\ell)}}^{(\ell)}(x) d \nu(x) \frac{<f_{\mathbf{m}^{(\ell)}}, g_{\mathbf{m}^{(\ell)}}>_{F}}{\left(\frac{d}{r}\right)_{\mathbf{m}^{(\ell)}}} .
$$

(ii) For any $\lambda>p_{\ell}-1$,

$$
<f, g>_{L^{2}\left(\mathcal{O}_{\ell}, \mu_{\lambda}^{(\ell)}\right)}=\sum_{\mathbf{m}^{(\ell)}} \frac{\left(\ell \frac{a}{2}\right)_{\mathbf{m}^{(\ell)}}\left(\frac{d^{(\ell)}}{\ell}\right)_{\mathbf{m}^{(\ell)}}}{\left(r \frac{a}{2}\right)_{\mathbf{m}^{(\ell)}}\left(\frac{d}{r}\right)_{\mathbf{m}^{(\ell)}}} \frac{<f_{\mathbf{m}^{(\ell)}}, g_{\mathbf{m}^{(\ell)}}>_{F}}{(\lambda)_{\mathbf{m}^{(\ell)}}} .
$$

(iii)

$$
<f, g>_{L^{2}\left(\mathcal{O}_{\ell}, \sigma_{\ell}\right)}=\sum_{\mathbf{m}^{(\ell)}} \frac{\left(\ell \frac{a}{2}\right)_{\mathbf{m}^{(\ell)}}}{\left(r \frac{a}{2}\right)_{\mathbf{m}^{(\ell)}}\left(\frac{d}{r}\right)_{\mathbf{m}^{(\ell)}}}<f_{\mathbf{m}^{(\ell)}}, g_{\mathbf{m}^{(\ell)}}>_{F} .
$$


Construction of $\hat{\mu}$ : The $K^{(\ell)}$-invariant measure $\mu$ on $\overline{D_{0}\left(v_{\ell}\right)}$ is used to define a measure $\hat{\mu}$ on $\partial_{r-\ell} D$ :

$$
\int_{\partial_{r-\ell} D} f d \hat{\mu}=\int_{\overline{D_{0}\left(v_{\ell}\right)}}\left(\int_{K} f\left(k\left(v_{\ell}+z\right)\right) d k\right) d \mu(z) .
$$

Obviously,

$$
\int_{\partial_{r-\ell} D} f d \hat{\mu}=\int_{\overline{I^{(\ell)}}}\left(\int_{K} f\left(k\left(v_{\ell}+x^{\frac{1}{2}}\right)\right) d k\right) d \nu(x)=\int_{I^{(\ell)}} \tilde{f}\left(v_{\ell}+x\right) d \nu(x) .
$$




\section{Integral Formulas for Invariant Inner Products}

\subsection{Discrete Wallach points for Cartan domains}

In this section we obtain the formulas for the inner products $<f, g>_{\ell \frac{a}{2}}, 1 \leq \ell \leq r-1$, via integration on the $K$-orbits $\partial_{r-\ell} D$ and $\mathcal{O}_{\ell}$.

Let $\left\{\sigma_{j}^{(\ell)}\right\}_{j=1}^{\ell}$ be the elementary symmetric polynomials $(1.2 .4)$ in the variables $\boldsymbol{\lambda}^{(\ell)}=$ $\left(\lambda_{1}, \lambda_{2}, \ldots, \lambda_{\ell}\right)$, and let $\boldsymbol{\sigma}^{(\ell)}$ be the vector map $\boldsymbol{\lambda}^{(\ell)} \mapsto\left(\sigma_{1}^{(\ell)}\left(\boldsymbol{\lambda}^{(\ell)}\right), \ldots, \sigma_{\ell}^{(\ell)}\left(\boldsymbol{\lambda}^{(\ell)}\right)\right)$. Following the remark after Lemma 1.2.5, let $\gamma^{(\ell)}: \mathcal{D}_{\ell} \rightarrow \mathcal{S}_{\ell}$ be the Harish-Chandra isomorphism, and let

$$
\Delta_{j}^{(\ell)}=\left(\gamma^{(\ell)}\right)^{-1}\left(\sigma_{j}^{(\ell)}\right), \quad 1 \leq j \leq \ell
$$

We define also $\sigma_{0}^{(\ell)}\left(\boldsymbol{\lambda}^{(\ell)}\right) \equiv 1, \Delta_{0}^{(\ell)}=I$, and let $W_{\ell}$ be the permutation group of the coordinates

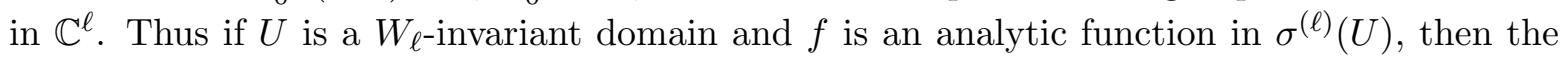
operator

$$
f\left(\Delta^{(\ell)}\right)=f\left(\Delta_{1}^{(\ell)}, \ldots, \Delta_{\ell}^{(\ell)}\right)
$$

(defined via the functional calculus analogous to Lemma 1.2.5) is $\operatorname{GL}\left(\Omega^{(\ell)}\right)$-invariant and satisfies

$$
f\left(\boldsymbol{\Delta}^{(\ell)}\right)\left(\Phi_{\boldsymbol{\lambda}^{(\ell)}+\boldsymbol{\rho}^{(\ell)}}^{(\ell)}\right)=f\left(\sigma^{(\ell)}\left(\boldsymbol{\lambda}^{(\ell)}\right)\right) \Phi_{\boldsymbol{\lambda}^{(\ell)}+\boldsymbol{\rho}^{(\ell)}}^{(\ell)}
$$

for every $\boldsymbol{\lambda}^{(\ell)} \in U$, where $\boldsymbol{\rho}^{(\ell)}$ is given by (1.2.13). In particular, for every partition $\mathbf{m}^{(\ell)}=$ $\left(m_{1}, \ldots, m_{\ell}, 0,0, \ldots, 0\right) \geq 0$ we obtain

$$
f\left(\boldsymbol{\Delta}^{(\ell)}\right)\left(\Phi_{\mathbf{m}^{(\ell)}}^{(\ell)}\right)=f\left(\sigma^{(\ell)}\left(\boldsymbol{\lambda}^{(\ell)}\right)\right) \Phi_{\mathbf{m}^{(\ell)}}^{(\ell)}
$$

where $\boldsymbol{\lambda}^{(\ell)}:=\mathbf{m}^{(\ell)}-\boldsymbol{\rho}^{(\ell)}$.

Lemma 2.1.1 Let $\alpha>(\ell-1) \frac{a}{2}$. Then for every partition $\mathbf{m}^{(\ell)}=\left(m_{1}, \ldots, m_{\ell}, 0,0, \ldots, 0\right)$ we have

$$
\Gamma_{\Omega^{(\ell)}}\left(\alpha+\mathbf{m}^{(\ell)}\right)=(2 \pi)^{\ell(\ell-1) \frac{a}{4}} \prod_{j=1}^{\ell} \Gamma\left(\lambda_{j}+\alpha-\frac{a}{4}(\ell-1)\right)
$$

where $\lambda_{j}:=m_{j}-\rho_{j}^{(\ell)}=m_{j}-(2 j-\ell-1) \frac{a}{4}$. Thus $\Gamma_{\Omega^{(\ell)}}\left(\alpha+\mathbf{m}^{(\ell)}\right)$ and $(\alpha)_{\mathbf{m}^{(\ell)}}=\Gamma_{\Omega^{(\ell)}}(\alpha+$ $\left.\mathbf{m}^{(\ell)}\right) / \Gamma_{\Omega^{(\ell)}}(\alpha)$ are symmetric functions of $\boldsymbol{\lambda}^{(\ell)}=\left(\lambda_{1}, \lambda_{2}, \ldots, \lambda_{\ell}\right)$. Moreover, for any $s \in \mathbb{N}$

$$
\begin{aligned}
\frac{(\alpha+s)_{\mathbf{m}^{(\ell)}}}{(\alpha)_{\mathbf{m}^{(\ell)}}} & =\prod_{\nu=0}^{s-1} \prod_{j=1}^{\ell}\left(\lambda_{j}+\alpha+\nu-\frac{a}{4}(\ell-1)\right) \\
& =\prod_{\nu=0}^{s-1} \sum_{k=0}^{\ell}\left(\alpha+\nu-\frac{a}{4}(\ell-1)\right)^{\ell-k} \sigma_{k}^{(\ell)}\left(\boldsymbol{\lambda}^{(\ell)}\right) .
\end{aligned}
$$

Thus $(\alpha+s)_{\mathbf{m}^{(\ell)}} /(\alpha)_{\mathbf{m}^{(\ell)}}$ is a symmetric polynomial in $\boldsymbol{\lambda}^{(\ell)}=\left(\lambda_{1}, \lambda_{2}, \ldots, \lambda_{\ell}\right)$. Hence the operator

$$
T:=\prod_{\nu=0}^{s-1} \sum_{k=0}^{\ell}\left(\alpha+\nu-\frac{a}{4}(\ell-1)\right)^{\ell-k} \Delta_{k}^{(\ell)}
$$


belongs to $\mathcal{D}_{\ell}$ and satisfies

$$
T f_{\mathbf{m}^{(\ell)}}=\frac{(\alpha+s)_{\mathbf{m}^{(\ell)}}}{(\alpha)_{\mathbf{m}^{(\ell)}}} f_{\mathbf{m}^{(\ell)}}
$$

for every $\mathbf{m}^{(\ell)}=\left(m_{1}, \ldots, m_{\ell}, 0, \ldots, 0\right) \geq 0$ and $f_{\mathbf{m}^{(\ell)}} \in P_{\mathbf{m}^{(\ell)}}$.

Proof: $(2.1 .5)$ is a consequence of $(1.1 .13)$ for the cone $\Omega^{(\ell)}$. The first equality in (2.1.6) is a consequence of (2.1.5) and the fact that $\Gamma(z+1)=z \Gamma(z)$, and the second is a well-known property of the $\left\{\sigma_{k}^{(\ell)}\right\}_{k=0}^{\ell}$. The rest follows from (2.1.3).

Remark 2.1.2 For every $\beta \in \mathbb{C}$ define

$$
D^{(\ell)}(\beta):=N_{\ell}^{\beta+1} \partial_{N_{\ell}} N_{\ell}^{-\beta} \in \mathcal{D}_{\ell}
$$

It is well-known (see [FK94] Chapter XIV and [AU97]) that

$$
\gamma_{D^{(\ell)}(\beta)}^{(\ell)}\left(\boldsymbol{\lambda}^{(\ell)}\right)=\prod_{j=1}^{\ell}\left(\lambda_{j}+\frac{a}{4}(\ell-1)-\beta\right) .
$$

It follows from (2.1.6) that if $\alpha>(\ell-1) \frac{a}{2}$ and $s \in \mathbb{N}$ then

$$
\frac{(\alpha+s)_{\mathbf{m}^{(\ell)}}}{(\alpha)_{\mathbf{m}^{(\ell)}}}=\prod_{\nu=0}^{s-1} \gamma_{D^{(\ell)}\left(\frac{a}{2}(\ell-1)-\alpha-\nu\right)}^{(\ell)}\left(\boldsymbol{\lambda}^{(\ell)}\right) .
$$

Since $\gamma^{(\ell)}: \mathcal{D}_{\ell} \rightarrow \mathcal{S}_{\ell}$ is a (surjective) ring isomorphism, it follows that the operator (2.1.7) admits the following expression

$$
T=N_{\ell}^{\frac{a}{2}(\ell-1)-\alpha} N_{\ell}\left(\frac{d}{d x}\right)^{s} N_{\ell}^{\alpha+s-\frac{a}{2}(\ell-1)} .
$$

Theorem 2.1.3 Let $0 \leq \ell \leq r-1$ and let $\beta>\frac{a}{2}(\ell-1)$. Then there exists an operator $T=T^{(\ell, \beta)}$ on $C^{\infty}\left(\Omega^{(\ell)}\right)$ which is invariant under $\mathrm{GL}\left(\Omega^{(\ell)}\right)$, so that for every $f \in \mathcal{H}_{\ell \frac{a}{2}}$ with Peter-Weyl expansion $f=\sum_{\mathbf{m}^{(\ell)}} f_{\mathbf{m}^{(\ell)}}$,

$$
T f=\sum_{\mathbf{m}^{(\ell)}} \frac{(\beta)_{\mathbf{m}^{(\ell)}}}{\left(\ell^{a}\right)_{\mathbf{m}^{(\ell)}}} f_{\mathbf{m}^{(\ell)}} .
$$

Hence, for all $f, g \in \mathcal{H}_{\ell \frac{a}{2}}$,

$$
<f, g>_{\ell \frac{a}{2}}=<T f, g>_{\beta} .
$$

Moreover, if $\beta-\ell \frac{a}{2} \in \mathbb{N}$ then $T \in \mathcal{D}_{\ell}$ (i.e. $T$ is a $\mathrm{GL}\left(\Omega^{(\ell)}\right)$-invariant differential operator).

Remark 2.1.4 Strictly speaking, the meaning of (2.1.13) is that $T^{\frac{1}{2}}$ (defined in general via the functional calculus $(1.2 .11)$, and for holomorphic functions via $T^{\frac{1}{2}}\left(\sum_{\mathbf{m}^{(\ell)}} f_{\mathbf{m}^{(\ell)}}\right)=$ $\left.\sum_{\mathbf{m}^{(\ell)}}\left(\frac{(\beta)_{\mathbf{m}^{(\ell)}}}{\left(\ell_{\frac{a}{2}}\right)_{\mathbf{m}}^{(\ell)}}\right)^{\frac{1}{2}} f_{\mathbf{m}^{(\ell)}}\right)$ maps $\mathcal{H}_{\ell_{\frac{2}{2}}}$ isometrically into $\mathcal{H}_{\beta}$. Formula $(2.1 .13)$ is valid for all polynomials $f, g$. 
Proof: We define an operator $T_{0}$ on holomorphic functions of the form $f=\sum_{\mathbf{m}^{(\ell)}} f_{\mathbf{m}^{(\ell)}}$ via $T_{0} f=\sum_{\mathbf{m}^{(\ell)}} \frac{(\beta) \mathbf{m}^{(\ell)}}{\left(\ell^{\frac{a}{2}}\right)_{\mathbf{m}^{(\ell)}}} f_{\mathbf{m}^{(\ell)}}$. Then $T_{0}$ is well defined and continuous with respect to the topology of uniform convergence on compact subsets of $D$ (see [A96]). Notice that the eigenvalues $(\beta)_{\mathbf{m}^{(\ell)}} /\left(\ell \frac{a}{2}\right)_{\mathbf{m}^{(\ell)}}$ are positive (since $m_{\ell+1}=\cdots=m_{r}=0$ and $\beta>\frac{a}{2}(\ell-1)$ ). If $f=\sum_{\mathbf{m}^{(\ell)}} f_{\mathbf{m}^{(\ell)}}$ and $g=\sum_{\mathbf{m}^{(\ell)}} g_{\mathbf{m}^{(\ell)}}$ are polynomials then

$$
<T f, g>_{\beta}=\sum_{\mathbf{m}^{(\ell)}} \frac{1}{\left(\ell_{\frac{a}{2}}\right)_{\mathbf{m}^{(\ell)}}}<f_{\mathbf{m}^{(\ell)}}, g_{\mathbf{m}^{(\ell)}}>_{F}=<f, g>_{\ell^{\frac{a}{2}}} .
$$

Thus $T_{0}^{\frac{1}{2}}$ maps $\mathcal{H}_{\ell \frac{a}{2}}$ into $\mathcal{H}_{\beta}$ isometrically. Using the notation $\boldsymbol{\lambda}^{(\ell)}=\mathbf{m}^{(\ell)}-\boldsymbol{\rho}^{(\ell)}$, Lemma 2.1.1 guarantees that there exists a symmetric function of $\boldsymbol{\lambda}^{(\ell)}$ of the form $p\left(\boldsymbol{\sigma}^{(\ell)}\left(\boldsymbol{\lambda}^{(\ell)}\right)\right)=$ $p\left(\sigma_{1}^{(\ell)}\left(\boldsymbol{\lambda}^{(\ell)}\right), \ldots, \sigma_{\ell}^{(\ell)}\left(\boldsymbol{\lambda}^{(\ell)}\right)\right)$, so that for all $\mathbf{m}^{(\ell)} \geq 0$

$$
\frac{(\beta)_{\mathbf{m}^{(\ell)}}}{\left(\ell \frac{a}{2}\right)_{\mathbf{m}^{(\ell)}}}=p\left(\boldsymbol{\sigma}^{(\ell)}\left(\boldsymbol{\lambda}^{(\ell)}\right)\right)
$$

Hence $T:=p\left(\boldsymbol{\Delta}^{(\ell)}\right)=p\left(\Delta_{1}^{(\ell)}, \ldots, \Delta_{\ell}^{(\ell)}\right)$ is a $\operatorname{GL}\left(\Omega^{(\ell)}\right)$-invariant operator whose restriction to the holomorphic functions of the form $\sum_{\mathbf{m}^{(\ell)}} f_{\mathbf{m}^{(\ell)}}$ is $T_{0}$. If $n:=\beta-\ell_{\frac{a}{2}} \in \mathbb{N}$, then (2.1.6) shows that $p$ is the polynomial

$$
p\left(x_{1}, \ldots, x_{\ell}\right)=\prod_{\nu=0}^{n-1}\left(\sum_{k=0}^{\ell}\left(\ell \frac{a}{2}+\nu-\frac{a}{4}(\ell-1)\right)^{\ell-k} x_{k}\right)
$$

where $x_{0}:=1$. Hence

$$
T=p\left(\Delta_{1}^{(\ell)}, \ldots, \Delta_{\ell}^{(\ell)}\right)=\prod_{\nu=0}^{n-1}\left(\sum_{k=0}^{\ell}\left(\ell \frac{a}{2}+\nu-\frac{a}{4}(\ell-1)\right)^{\ell-k} \Delta_{k}^{(\ell)}\right)
$$

is a member of $\mathcal{D}_{\ell}$ (i.e. a polynomial in the generators $\Delta_{1}^{(\ell)}, \ldots, \Delta_{\ell}^{(\ell)}$ ).

Using (2.1.4) and Corollary 1.3.6 we obtain the following result.

Corollary 2.1.5 Let $f, g \in \mathcal{H}_{\ell \frac{a}{2}}$ have Peter-Weyl expansions $f=\sum_{\mathbf{m}^{(\ell)}} f_{\mathbf{m}^{(\ell)}}$ and $g=$ $\sum_{\mathbf{m}^{(\ell)}} g_{\mathbf{m}^{(\ell)}}$. Then for every symmetric function of $\boldsymbol{\lambda}^{(\ell)}$ of the form

$$
p\left(\boldsymbol{\sigma}^{(\ell)}\left(\boldsymbol{\lambda}^{(\ell)}\right)\right)=p\left(\sigma_{1}^{(\ell)}\left(\boldsymbol{\lambda}^{(\ell)}\right), \ldots, \sigma_{\ell}^{(\ell)}\left(\boldsymbol{\lambda}^{(\ell)}\right)\right)
$$

the corresponding differential operator

$$
p\left(\boldsymbol{\Delta}^{(\ell)}\right)=p\left(\Delta_{1}^{(\ell)}, \ldots, \Delta_{\ell}^{(\ell)}\right) \in \mathcal{D}_{\ell}
$$

satisfies for every $x \in \Omega^{(\ell)}$ :

$$
\begin{aligned}
& \left(p\left(\boldsymbol{\Delta}^{(\ell)}\right)(\widetilde{f \bar{g}})\right)(x)= \\
& \quad=c_{\ell} \sum_{\mathbf{m}^{(\ell)}} \frac{\left(\ell \frac{a}{2}\right)_{\mathbf{m}^{(\ell)}}}{\left(r \frac{a}{2}\right)_{\mathbf{m}^{(\ell)}}\left(\frac{d}{r}\right)_{\mathbf{m}^{(\ell)}}} p\left(\boldsymbol{\sigma}^{(\ell)}\left(\mathbf{m}^{(\ell)}-\boldsymbol{\rho}^{(\ell)}\right)\right)<f_{\mathbf{m}^{(\ell)}}, g_{\mathbf{m}^{(\ell)}}>_{F} \Phi_{\mathbf{m}^{(\ell)}}^{(\ell)}(x) .
\end{aligned}
$$


If $\alpha>p_{\ell}-1=(\ell-1) a+1+b$, then

$$
\begin{aligned}
& \int_{I_{\ell}} p\left(\boldsymbol{\Delta}^{(\ell)}\right)(\widetilde{f \bar{g}}) d \nu_{\alpha}^{(\ell)}= \\
& \quad=c_{\ell} \sum_{\mathbf{m}^{(\ell)}} \frac{\left(\ell \frac{a}{2}\right)_{\mathbf{m}^{(\ell)}}\left(\frac{d_{\ell}}{\ell}\right)_{\mathbf{m}^{(\ell)}}}{\left(r \frac{a}{2}\right)_{\mathbf{m}^{(\ell)}}\left(\frac{d}{r}\right)_{\mathbf{m}^{(\ell)}}(\alpha)_{\mathbf{m}^{(\ell)}}} p\left(\boldsymbol{\sigma}^{(\ell)}\left(\mathbf{m}^{(\ell)}-\boldsymbol{\rho}^{(\ell)}\right)\right)<f_{\mathbf{m}^{(\ell)}}, g_{\mathbf{m}^{(\ell)}}>_{F} .
\end{aligned}
$$

Here $c_{\ell}=\Gamma_{\Omega^{(\ell)}}\left(\ell \frac{a}{2}\right) / \Gamma_{\Omega^{(\ell)}}\left(r \frac{a}{2}\right), d_{\ell}=\operatorname{dim}_{\mathbb{R}}\left(X_{1}\left(u_{\ell}\right)\right)=\ell(\ell-1) \frac{a}{2}+1$, and $\nu_{\alpha}^{(\ell)}$ is the measure defined in (1.4.6).

Notice that by Lemma 2.1.1 the coefficients of $<f_{\mathbf{m}^{(\ell)}}, g_{\mathbf{m}^{(\ell)}}>_{F} \Phi_{\mathbf{m}^{(\ell)}}^{(\ell)}(x)$ in $(2.1 .14)$ and (2.1.15) are symmetric functions of $\boldsymbol{\lambda}^{(\ell)}:=\mathbf{m}^{(\ell)}-\boldsymbol{\rho}^{(\ell)}$.

Remark 2.1.6 (i) If one uses (2.1.14) with $x=u_{\ell}$, one obtains (with $T=p\left(\boldsymbol{\Delta}^{(\ell)}\right)$ )

$$
\begin{aligned}
& (T \tilde{f} \cdot \bar{g})\left(u_{\ell}\right)=T(\widetilde{f \cdot \bar{g}})\left(u_{\ell}\right)= \\
& \quad=c_{\ell} \sum_{\mathbf{m}^{(\ell)}} \frac{\left(\ell \frac{a}{2}\right)_{\mathbf{m}^{(\ell)}} t_{\mathbf{m}^{(\ell)}}}{\left(r \frac{a}{2}\right)_{\mathbf{m}^{(\ell)}}\left(\frac{d}{r}\right)_{\mathbf{m}^{(\ell)}}}<f_{\mathbf{m}^{(\ell)}}, g_{\mathbf{m}^{(\ell)}}>_{F} .
\end{aligned}
$$

(ii) If we choose $T$ so that its eigenvalues satisfy

$$
c_{\ell} \frac{\left(\ell \frac{a}{2}\right)_{\mathbf{m}^{(\ell)}} t_{\mathbf{m}^{(\ell)}}}{\left(r \frac{a}{2}\right)_{\mathbf{m}^{(\ell)}}\left(\frac{d}{r}\right)_{\mathbf{m}^{(\ell)}}}=\frac{1}{\left(\ell_{\frac{a}{2}}\right)_{\mathbf{m}^{(\ell)}}}
$$

then for every $f, g \in \mathcal{H}_{\ell \frac{a}{2}}$

$$
\widetilde{T f \cdot \bar{g}}\left(u_{\ell}\right)=\sum_{\mathbf{m}^{(\ell)}} \frac{<f_{\mathbf{m}^{(\ell)}}, g_{\mathbf{m}^{(\ell)}}>_{F}}{\left(\ell_{\frac{a}{2}}\right)_{\mathbf{m}^{(\ell)}}}=<f, g>_{\ell \frac{a}{2}} .
$$

Namely

$$
<f, g>_{\ell \frac{a}{2}}=\int_{S_{\ell}}(T f \cdot \bar{g})(v) d \sigma_{\ell}(v) .
$$

This realizes $\mathcal{H}_{\ell \frac{a}{2}}$ as a Hardy-type space on $S_{\ell}$

(iii) It would be interesting to exhibit $T$ in concrete terms (not only via its eigenvalues). If $a$ is even then $T \in \mathcal{D}_{\ell}$, i.e. $T$ is a polynomial in the generators $\Delta_{1}^{(\ell)}, \Delta_{2}^{(\ell)}, \ldots, \Delta_{\ell}^{(\ell)}$ of $\mathcal{D}_{\ell}$. It would be interesting also to exhibit $T$ as a linear combination of Yan's operators (see [AU97]). If $a$ is odd then either $D$ is of type $I V_{n}$ with $n$ odd (a case which was considered in [AU97] and [AU98] since $\ell=1$ ), or $D$ is of type $I I I_{r}$ (with $a=1$ ).

Theorem 2.1.7 Let $0 \leq \ell \leq r-1$ and let $\alpha>p_{\ell}-1=(\ell-1) a+1+b$. Let $p\left(\sigma^{(\ell)}\left(\boldsymbol{\lambda}^{(\ell)}\right)\right)$ be the symmetric function of $\boldsymbol{\lambda}^{(\ell)}=\left(\lambda_{1}, \lambda_{2}, \ldots, \lambda_{\ell}\right)$ so that

$$
p\left(\sigma^{(\ell)}\left(\boldsymbol{\lambda}^{(\ell)}\right)\right)=\frac{1}{c_{\ell}} \frac{\left(r \frac{a}{2}\right)_{\mathbf{m}^{(\ell)}\left(\frac{d}{r}\right)} \mathbf{m}^{(\ell)}(\alpha)_{\mathbf{m}^{(\ell)}}}{\left(\ell_{2}^{a}\right)_{\mathbf{m}^{(\ell)}}^{2}\left(\frac{d_{\ell}}{\ell}\right)_{\mathbf{m}^{(\ell)}}}
$$


for every $\boldsymbol{\lambda}^{(\ell)}:=\mathbf{m}^{(\ell)}-\boldsymbol{\rho}^{(\ell)}$. Let $T=p\left(\Delta_{1}^{(\ell)}, \ldots, \Delta_{\ell}^{(\ell)}\right)$ be the $\operatorname{GL}\left(\Omega^{(\ell)}\right)$-invariant operator defined via the functional calculus (Lemma 1.2.5). Then for every $f, g \in \mathcal{H}_{\ell \frac{a}{2}}$

$$
<f, g>_{\ell \frac{a}{2}}=\int_{I_{\ell}} T(\widetilde{f \bar{g}}) d \nu_{\alpha}^{(\ell)}
$$

where $\nu_{\alpha}^{(\ell)}$ is the measure defined in (1.4.6). Moreover, if $s:=\alpha-\ell \frac{a}{2} \in \mathbb{N}$, then $p$ is a polynomial in $\boldsymbol{\lambda}^{(\ell)}$ and $T \in \mathcal{D}_{\ell}$, i.e. $T$ is a $\mathrm{GL}\left(\Omega^{(\ell)}\right)$-invariant differential operator on $\Omega$.

Proof: The right hand side of (2.1.18) is symmetric in $\boldsymbol{\lambda}^{(\ell)}:=\mathbf{m}^{(\ell)}-\boldsymbol{\rho}^{(\ell)}$ by Lemma 2.1.1. Thus (2.1.14) yields for any $f, g \in \mathcal{H}_{\ell \frac{a}{2}}$ with Peter-Weyl expansions $f=\sum_{\mathbf{m}^{(\ell)}} f_{\mathbf{m}^{(\ell)}}$ and $g=\sum_{\mathbf{m}^{(\ell)}} g_{\mathbf{m}^{(\ell)}}$,

$$
\begin{aligned}
\int_{I_{\ell}} T(\widetilde{f \bar{g}}) d \nu_{\alpha}^{(\ell)} & =\sum_{\mathbf{m}^{(\ell)}} \frac{<f_{\mathbf{m}^{(\ell)}}, g_{\mathbf{m}^{(\ell)}>_{F}}(\alpha)_{\mathbf{m}^{(\ell)}}}{\left(\ell_{\frac{a}{2}}\right)_{\mathbf{m}^{(\ell)}}\left(\frac{d_{\ell}}{\ell}\right)_{\mathbf{m}^{(\ell)}}} \int_{I_{\ell}} \Phi_{\mathbf{m}^{(\ell)}}^{(\ell)}(x) d \nu_{\alpha}^{(\ell)}(x) \\
& =\sum_{\mathbf{m}^{(\ell)}} \frac{<f_{\mathbf{m}^{(\ell)}}, g_{\mathbf{m}^{(\ell)}}>_{F}}{\left(\ell_{\frac{a}{2}}\right)_{\mathbf{m}^{(\ell)}}}=<f, g>_{\ell \frac{a}{2}} .
\end{aligned}
$$

Assume that $s:=\alpha-\ell \frac{a}{2} \in \mathbb{N}$. If also $n:=(r-\ell) \frac{a}{2} \in \mathbb{N}$ then

$$
r \frac{a}{2}-\ell \frac{a}{2}=\frac{d}{r}-\frac{d_{\ell}}{\ell}=n,
$$

and Lemma 2.1.1 guarantees that $p$ is a symmetric polynomial of degree $\ell(s+2 n)$ in $\left(\lambda_{1}, \lambda_{2}, \ldots, \lambda_{\ell}\right)$. If $(r-\ell) \frac{a}{2} \notin \mathbb{N}$, then necessarily $b=0$, and both

$$
n_{1}:=(r-\ell+1) \frac{a}{2}-1 \quad \text { and } \quad n_{2}:=\frac{d}{r}-\ell \frac{a}{2}=(r-1-\ell) \frac{a}{2}+1
$$

are in $\mathbb{N}$. Again, Lemma 2.1.1 guarantees that $p$ is a polynomial of degree $\ell\left(s+n_{1}+n_{2}\right)$ in $\left(\lambda_{1}, \lambda_{2}, \ldots, \lambda_{\ell}\right)$. This completes the proof.

Remark 2.1.8 (i) Using Lemma 1.3.1 and Proposition 1.3.2 it follows that if $T$ is a $\operatorname{GL}\left(\Omega^{(\ell)}\right)$ invariant operator on $C^{\infty}\left(\Omega^{(\ell)}\right)$, then for every $f, g \in \mathcal{H}_{\ell \frac{a}{2}}$

$$
T(\widetilde{f \bar{g}})=\widetilde{T f \cdot \bar{g}}=\widetilde{f \cdot \overline{T g}} .
$$

Theorems 2.1.3 and 2.1.7 can be reformulated accordingly. For instance, (2.1.19) can be rewritten as

$$
<f, g>_{\ell \frac{a}{2}}=\int_{I_{\ell}} \widetilde{T f \cdot \bar{g}} d \nu_{\alpha}^{(\ell)}=\int_{I_{\ell}} \widetilde{f \cdot \overline{T g}} d \nu_{\alpha}^{(\ell)} .
$$

(ii) Formula (2.1.18) can be rewritten as

$$
<f, g>_{\ell \frac{a}{2}}=\int_{K}\left(\int_{D_{0}\left(v_{\ell}\right)}(T f \cdot \bar{g})(k(z)) d \mu_{\alpha}^{(\ell)}(z)\right) d k
$$




\subsection{Discrete Wallach points for symmetric tube domains using Fourier trans- form}

In the following we develop integral formulas for the inner products in the spaces $\mathcal{H}_{\ell \frac{a}{2}}(T(\Omega))$, (where $T(\Omega)$ is the symmetric Siegel associated to $D$ via the Cayley transform) in terms of the Fourier transform of the functions. We begin with the relatively simple case of a Siegel domain of type $I$. The results presented below for the discrete Wallach points $\left\{\ell_{2} \frac{a}{2}\right\}_{\ell=0}^{r-1}$ will be somewhat analogous to our earlier results [AU97] for the continuous Wallach points $\lambda>(r-1) \frac{a}{2}$. The development of the integral formulas for a general symmetric Siegel domain of type $I I$ requires additional machinery, and will be treated in the next section.

Assume that $Z$ is a $J B^{*}$-algebra with a unit $e$. The open unit ball of $Z$ is holomorphically equivalent to the tube domain

$$
T(\Omega)=X+i \Omega
$$

via the Cayley transform $c(z)=i(e+z)(e-z)^{-1}, z \in D . T(\Omega)$ is a symmetric Siegel domain of type $I$. For any $\lambda \in W(D)$ the operator $V^{(\lambda)} f=\left(f \circ c^{-1}\right)\left(J c^{-1}\right)^{\lambda / p}$ maps the space $\mathcal{H}_{\lambda}(\mathcal{D})$ isometrically onto a Hilbert space of analytic functions on $T(\Omega)$, denoted by $\mathcal{H}_{\lambda}(T(\Omega))$. The inner product in $\mathcal{H}_{\lambda}(T(\Omega))$ is defined by

$$
\langle f, g\rangle_{\lambda}=\langle f, g\rangle_{\mathcal{H}_{\lambda}(T(\Omega))}=\left\langle V^{(\lambda)^{-1}}(f), V^{(\lambda)^{-1}} g\right\rangle_{\mathcal{H}_{\lambda}(\mathcal{D})} .
$$

The description of $\mathcal{H}_{\lambda}(T(\Omega))$ is therefore equivalent to the description of $\mathcal{H}_{\lambda}(\mathcal{D})$.

The reproducing kernel of $\mathcal{H}_{\lambda}(T(\Omega))$ is

$$
K_{\lambda}(z, w)=N\left(\frac{z-w^{*}}{i}\right)^{-\lambda}, \quad z, w \in T(\Omega) .
$$

Namely, for all $z, w \in T(\Omega)$

$$
\left(J\left(c^{-1}\right)(z)^{\lambda / p} h\left(c^{-1}(z), c^{-1}(w)\right)^{-\lambda}{\overline{\left(J c^{-1}(w)\right.}}^{\lambda / p}=N\left(\frac{z-w^{*}}{i}\right)^{-\lambda} .\right.
$$

It is known that for $\lambda>p-1 \mathcal{H}_{\lambda}(T(\Omega))$ is the weighted Bergman space

$$
\mathcal{H}_{\lambda}(T(\Omega))=L_{a}^{2}\left(T(\Omega), m_{\lambda}\right)=L^{2}\left(T(\Omega), m_{\lambda}\right) \cap\{\text { analytic function }\}
$$

where

$$
d m_{\lambda}(z)=c_{\lambda} d x N(2 y)^{\lambda-p} d y, \quad z=x+i y, x \in X, y \in \Omega
$$

and

$$
c_{\lambda}=\frac{\Gamma_{\Omega}(\lambda)}{\pi^{d} \Gamma_{\Omega}\left(y-\frac{d}{r}\right)}
$$

Also, the Shilov boundary of $T(\Omega)$ is $X:=\left\{z \in Z ; z^{*}=z\right\}$, and $\mathcal{H}_{\frac{d}{r}}(T(\Omega))$ coincides with the Hardy space $H^{2}(X)$ (consisting of all analytic functions $f$ in $T(\Omega)$ for which $\|f\|_{H^{2}(X)}^{2}:=$ $\left.\sup _{y \in \Omega} \int_{X}|f(x+i y)|^{2} d x<\infty\right)$.

Using the Fourier transform (with respect to $x$ ) one obtains the following result. Here for $\lambda>(r-1) \frac{g}{2}$ we consider on $\Omega$ the measure

$$
d \sigma_{\lambda}(v)=\beta_{\lambda} N(v)^{\frac{d}{r}-\lambda} d v, \quad \beta_{\lambda}=(2 \pi)^{-2 d} \Gamma_{\Omega}(\lambda) .
$$


Proposition 2.2.1 [AU97; Proposition 6.1] Let $\lambda>(r-1) \frac{a}{2}$, and let $f$ be a holomorphic function in $T(\Omega)$. Then the following are equivalent:

(i) $f \in \mathcal{H}_{\lambda}(T(\Omega))$;

(ii) The boundary values $f(x):=\lim _{\Omega \ni y \rightarrow 0} f(x+i y)$ exist almost everywhere on $X$, and the Fourier transform $\hat{f}$ of $f(x)$ is supported in $\bar{\Omega}$ and belongs to $L^{2}\left(\Omega, \sigma_{\lambda}\right)$;

Moreover, the map $f \mapsto \hat{f}$ is an isometry of $\mathcal{H}_{\lambda}(T(\Omega))$ onto $L^{2}\left(\Omega, \sigma_{\lambda}\right)$. Consequently, for all $f, g \in \mathcal{H}_{\lambda}(T(\Omega))$

$$
\langle f, g\rangle_{\lambda}=\int_{\Omega} \hat{f} \overline{\hat{g}} d \sigma_{\lambda}
$$

Our goal here is to extend Proposition 2.2.1 to the discrete Wallach points $\ell \frac{a}{2}, \ell=0,1, \ldots, r-$ 1. With respect to the fixed frame $\left\{e_{j}\right\}_{j=1}^{r}$ of minimal, pairwise orthogonal idempotents, we denote $u_{\ell}=\sum_{j=1}^{\ell} e_{j}, v_{\ell}=\sum_{j=\ell+1}^{r} e_{j}, 0 \leq \ell \leq r-1$. Recall that the orbits of $\operatorname{GL}(\Omega)$ on $\partial \Omega$ are

$$
\begin{aligned}
\partial_{\ell} \Omega & =\operatorname{GL}(\Omega)\left(u_{\ell}\right)=\left\{\varphi\left(u_{\ell}\right) ; \varphi \in \mathrm{GL}(\Omega)\right\} \\
& =\{x \in \bar{\Omega} ; \operatorname{rank}(x)=\ell\}, \ell=0,1,2, \ldots, r-1
\end{aligned}
$$

The following fundamental fact is established in [RV76] and [La87]. An alternative direct proof will be given in Section 3.1 below.

Theorem 2.2.2 Let $0 \leq \ell \leq r-1$. There exists a unique measure $\mu_{\ell}$ on $\partial_{\ell} \Omega$, having the following properties:

$$
d \mu_{\ell}(\varphi(x))=\operatorname{Det}(\varphi)^{\ell \frac{a}{2} / \frac{d_{1}}{r}} d \mu_{\ell}(x), \quad \forall \varphi \in G L(\Omega)
$$

where $d_{1}=\operatorname{dim}\left(Z_{1}(e)\right)=r(r-1) \frac{a}{2}+r$, and

$$
\int_{\partial_{\ell} \Omega} e^{-<x, y>} d \mu_{\ell}(y)=\gamma_{\ell} N_{\ell}(x)^{-\ell \frac{a}{2}} \quad \forall x \in \Omega,
$$

where $\gamma_{\ell}=(2 \pi)^{\ell(r-\ell) \frac{a}{2}} \Gamma_{\Omega^{(\ell)}}\left(\ell \frac{a}{2}\right)$.

Let $\mathrm{GL}(\Omega)=L N_{\Omega} A$ be the Iwasawa decomposition. Then it is known that the set

$$
N_{\Omega} A\left(u_{\ell}\right)=\left\{x \in \partial_{\ell}(\Omega) ; N_{\ell}(x)>0\right\}
$$

is open and dense in $\partial_{\ell} \Omega$ and $\mu_{\ell}\left(\partial_{\ell} \Omega \backslash N_{\Omega} A\left(u_{\ell}\right)\right)=0$. The following result is established in [La87].

Lemma 2.2.3 An element $x \in \partial_{\ell} \Omega$ belongs to $N_{\Omega} A\left(u_{\ell}\right)$ if and only if in its Peirce decomposition relative to $u_{\ell}: x=x_{1}+x_{1 / 2}+x_{0}, x_{1}$ is invertible in $X_{1}\left(u_{\ell}\right)$ and

$$
x_{0}=2 v_{\ell}\left(x_{1 / 2}\left(x_{1 / 2} x_{1}^{-1}\right)\right)
$$


The expression of $\mu_{\ell}$ in the coordinates $\left(x_{1}, x_{1 / 2}\right)$ of $x \in N_{\Omega} A\left(u_{\ell}\right)$ is

$$
d \mu_{\ell}(x)=N_{\ell}\left(x_{1}\right)^{\ell \frac{a}{2}-\frac{d}{r}} d x_{1} d x_{1 / 2}
$$

The properties of $\mu_{\ell}$ enable us to describe the space $\mathcal{H}_{\ell \frac{a}{2}}$.

Lemma 2.2.4 Fix $w \in T(\Omega)$ and $0 \leq \ell \leq r-1$. Then the Fourier transform with respect to $x$ of the function $K_{w}^{\left(\ell \frac{a}{2}\right)}(x)=K^{\left(\ell \frac{a}{2}\right)}(x, w)=N\left(\frac{x-w^{*}}{i}\right)^{-\ell \frac{a}{2}}$ is the following measure with support $\partial_{\ell} \Omega$ :

$$
\widehat{K_{w}^{\left(\ell \frac{a}{2}\right)}}(t)=\frac{(2 \pi)^{d}}{\gamma_{\ell}} e^{-i\left\langle w^{*} \mid t\right\rangle} d \mu_{\ell}(t)
$$

where $\gamma_{\ell}=(2 \pi)^{\ell(r-\ell) \frac{a}{2}} \Gamma_{\Omega(\ell)}\left(\frac{a}{2}\right)$, as in Theorem 2.2.2.

Proof: Theorem 2.2.2 and the fact that $\Omega$ is a set of uniqueness for holomorphic functions on $T(\Omega)$ imply that for all $z \in T(\Omega)$

$$
\int_{\partial_{\ell} \Omega} e^{i\langle z \mid t\rangle} d \mu_{\ell}(t)=\gamma_{\ell} N\left(\frac{z}{i}\right)^{\ell \frac{a}{2}}
$$

It follows that for all $z, w \in T(\Omega)$

$$
K^{\left(\ell \frac{a}{2}\right)}(z, w)=\left(N\left(\frac{z-w^{*}}{i}\right)\right)^{-\ell \frac{a}{2}}=\frac{1}{\gamma_{\ell}} \int_{\Omega_{\ell}} e^{-\left\langle\frac{z-w^{*}}{i} \mid t\right\rangle} d \mu_{\ell}(t)
$$

Hence

$$
K_{w}^{\left(\ell \frac{a}{2}\right)}(x)=\frac{1}{\gamma_{\ell}} \int_{\partial_{\ell} \Omega} e^{i\langle x \mid t\rangle} e^{-i\left\langle w^{*} \mid t\right\rangle} d \mu_{\ell}(t), \quad w \in T(\Omega)
$$

Thus $K_{w}^{\left(\ell \frac{a}{2}\right)}(x)$ is the inverse Fourier transform of the measure $\frac{(2 \pi)^{d}}{\gamma_{\ell}} e^{-i\left\langle w^{*} \mid t\right\rangle} d \mu_{\ell}(t)$, which is supported on $\partial_{\ell} \Omega$, and (2.2.11) follows.

Lemma 2.2 .4 can be reformulated by saying that $\widehat{K_{w}^{\left(\ell \frac{a}{2}\right)}}$ is a measure supported in $\partial_{\ell} \Omega$ which is absolutely continuous with respect to $\mu_{\ell}$, with Radon-Nikodym derivative

$$
\frac{d \widehat{K_{w}^{\left(\ell \frac{a}{2}\right)}}}{d \mu_{\ell}}(t)=\frac{(2 \pi)^{d}}{\gamma_{\ell}} e^{-i\left\langle w^{*} \mid t\right\rangle}
$$

Lemma 2.2.5 For every $z, w \in T(\Omega)$ and $0 \leq \ell \leq r-1$

$$
\left\langle\frac{d \widehat{K_{w}^{\left(\ell \frac{a}{2}\right)}}}{d \mu_{\ell}}, \frac{d \widehat{K_{z}^{\left(\ell \frac{a}{2}\right)}}}{d \mu_{\ell}}\right\rangle_{L^{2}\left(\partial_{\ell} \Omega, \mu_{\ell}\right)}=\frac{(2 \pi)^{2 d}}{\gamma_{\ell}} K^{\left(\ell \frac{a}{2}\right)}(z, w)
$$

Proof: Both sides of (2.2.14) are holomorphic in $z$ and anti-holomorphic in $w$. Therefore it suffices to prove (2.2.14) for $z=w=u+i v, u \in X, v \in \Omega$. In this case we obtain by Lemma 2.2 .3

$$
\begin{aligned}
\left\|\frac{d \widehat{K^{\left(\ell \frac{a}{2}\right)}}}{d \mu_{\ell}}\right\|_{L^{2}\left(\partial_{\ell} \Omega, \mu_{\ell}\right)}^{2} & =\frac{(2 \pi)^{2 d}}{\gamma_{\ell}^{2}} \int_{\partial_{\ell} \Omega}\left|e^{-i\left\langle w^{*} \mid t\right\rangle}\right|^{2} d \mu_{\ell}(t)=\frac{(2 \pi)^{2 d}}{\gamma_{\ell}^{2}} \int_{\partial_{\ell} \Omega} e^{-i\langle 2 v \mid t\rangle} d \mu_{\ell}(t) \\
& =\frac{(2 \pi)^{2 d}}{\gamma_{\ell}} N(2 v)^{-\ell \frac{a}{2}}=\frac{(2 \pi)^{2 d}}{\gamma_{\ell}} K^{\left(\ell \frac{a}{2}\right)}(w, w) .
\end{aligned}
$$


Fix $0 \leq \ell \leq r-1$ and consider the space

$$
\mathcal{H}_{\ell \frac{a}{2}}^{(0)}(T(\Omega)):=\operatorname{span}\left\{K_{w}^{\left(\ell \frac{a}{2}\right)} ; w \in T(\Omega)\right\} .
$$

We define a map $V_{\ell}^{(0)}$ on $\mathcal{H}_{\ell \frac{a}{2}}^{(0)}(T(\Omega))$ via

$$
V_{\ell}^{(0)} f=\frac{\gamma_{\ell}^{\frac{1}{2}}}{(2 \pi)^{d}} \frac{d \hat{f}}{d \mu_{\ell}}
$$

where $\hat{f}$ is the Fourier transform of the restriction of $f$ to the Shilov boundary $X$, and $\frac{d \hat{f}}{d \mu_{\ell}}$ is the Radon-Nikodym derivative of the measure $\hat{f}$ with respect to $\mu_{\ell}$, which exists in view of Lemma 2.2.4 and the fact that $f \in \mathcal{H}_{\ell \frac{a}{2}}^{(0)}(T(\Omega))$.

Lemma 2.2.6 $V_{\ell}^{(0)}$ is an isometry of $\mathcal{H}_{\ell \frac{a}{2}}^{(0)}(T(\Omega))$ into $L^{2}\left(\partial_{\ell} \Omega, \mu_{\ell}\right)$, and it has a dense range.

Proof: : Let $f=\sum_{j=1}^{n} c_{j} K_{w_{j}}^{\left(\ell \frac{a}{2}\right)} \in \mathcal{H}_{\ell \frac{a}{2}}^{(0)}(T \Omega)$. Then

$$
\|f\|_{\ell \frac{a}{2}}^{2}=\sum_{i, j=1}^{n} c_{i} \bar{c}_{j}\left\langle K_{w_{i}}^{\left(\ell \frac{a}{2}\right)}, K_{w_{j}}^{\ell \frac{a}{2}}\right\rangle_{\ell \frac{a}{2}}=\sum_{i, j=1}^{n} c_{i} \bar{c}_{j} K^{\left(\ell \frac{a}{2}\right)}\left(w_{j}, w_{i}\right) .
$$

Also, Lemma 2.2.5 implies

$$
\begin{aligned}
\left\|V_{\ell}^{(0)}\right\|_{L^{2}\left(\partial_{\ell} \Omega \mu_{\ell}\right)}^{2} & =\frac{\gamma_{\ell}}{(2 \pi)^{2 d}} \sum_{i, j=1}^{n} c_{i} \bar{c}_{j}\left\langle\frac{d \widehat{K_{w_{i}}^{\left(\ell \frac{a}{2}\right)}}}{d \mu_{\ell}}, \frac{d \widehat{K_{w_{j}}^{\left(\ell \frac{a}{2}\right)}}}{d \mu_{\ell}}\right\rangle_{L^{2}\left(\partial_{\ell} \Omega, \mu_{\ell}\right)} \\
& =\sum_{i, j=1}^{n} c_{i} \bar{c}_{j} K^{\left(\ell \frac{a}{2}\right)}\left(w_{j}, w_{i}\right)=\|f\|_{\ell \frac{a}{2}}^{2}
\end{aligned}
$$

Thus $V_{\ell}^{(0)}$ is an isometry. The range of $V_{\ell}^{(0)}$ contains all the functions

$$
V_{\ell}^{(0)}\left(\gamma_{\ell}^{1 / 2} K_{w}^{\left(\ell \frac{a}{2}\right)}\right)(t)=e^{-i\left\langle w^{*} \mid t\right\rangle}, w \in T(\Omega) .
$$

The linear span of these functions is a self-adjoint sub-algebra of $C\left(\partial_{\ell} \Omega\right)$, which separates the points of $\partial_{\ell} \Omega$. Therefore $V_{\ell}^{(0)}\left(\mathcal{H}_{\ell_{2}^{a}}^{(0)}(T(\Omega))\right.$ is dense in $C_{0}\left(\partial_{\ell} \Omega\right)$ by the Stone-Weierstrass theorem. Since $\mu_{\ell}$ is mutually absolutely continuous with respect to Lebesgue measure on $\partial_{\ell} \Omega$, the density of $V_{\ell}^{(0)}\left(\mathcal{H}_{\ell \frac{a}{2}}^{(0)}(T(\Omega))\right.$ in $L^{2}\left(\partial_{\ell} \Omega, \mu_{\ell}\right)$ follows now by standard arguments.

It follows from Lemma 2.2.6 that $V_{\ell}^{(0)}$ extends an isometry $V_{\ell}$ of $\mathcal{H}_{\ell \frac{a}{2}}^{(0)}(T(\Omega))$ onto $L^{2}\left(\partial_{\ell} \Omega, \mu_{\ell}\right)$. The exact statement is the following result.

Theorem 2.2.7 Let $0 \leq \ell \leq r-1$, and let $f$ be a holomorphic function in $T(\Omega)$. The following conditions are equivalent:

(i) $f \in \mathcal{H}_{\ell \frac{a}{2}}(T(\Omega))$; 
(ii) The boundary values $f(x)=\lim _{\Omega \ni y \rightarrow 0} f(x+i y)$ exist almost everywhere on $X$, the Fourier transform $\hat{f}$ of $f(x)$ is a measure with support in $\partial_{\ell} \Omega$ which is absolutely continuous with respect to $\mu_{\ell}$, and the Radon-Nikodym derivative $\frac{d \hat{f}}{d \mu_{\ell}}$ belongs to $L^{2}\left(\partial_{\ell} \Omega, \mu_{\ell}\right)$. Moreover, the map $V_{\ell} f=\frac{d \hat{f}}{d \mu_{\ell}}$ is an isometry of $\mathcal{H}_{\ell \frac{a}{2}}(T(\Omega))$ onto $L^{2}\left(\partial_{\ell} \Omega, \mu_{\ell}\right)$. Thus for all $f, g \in \mathcal{H}_{\ell \frac{a}{2}}^{(0)}(T(\Omega))$

$$
\langle f, g\rangle_{\ell \frac{a}{2}}=\frac{\Gamma_{\Omega(\ell)}\left(\ell \frac{a}{2}\right)}{(2 \pi)^{2 \delta_{\ell}}} \int_{\partial_{\ell} \Omega} \frac{d \hat{f}}{d \mu_{\ell}}(t) \frac{\overline{d \hat{g}}}{d \mu_{\ell}}(t) d \mu_{\ell}(t)
$$

where $\delta_{\ell}=d-\ell(r-\ell) \frac{a}{2}$.

Expressing $\mu_{\ell}$ via $(2.2 .10)$ on $N_{\Omega} A\left(u_{\ell}\right)$, we obtain

$$
\langle f, g\rangle_{\ell \frac{a}{2}}=\frac{\Gamma_{\Omega(\ell)}\left(\ell \frac{a}{2}\right)}{(2 \pi)^{2 \delta_{\ell}}} \int_{N_{\Omega} A\left(u_{\ell}\right)} \frac{d \hat{f}}{d \mu_{\ell}}(t) \overline{\frac{d \hat{g}}{d \mu_{\ell}}}(t) N_{\ell}\left(t_{1}\right)^{\ell \frac{a}{2}-\frac{d}{r}} d t_{1} d t_{2}
$$

Remark 2.2.8 In the case where $\lambda>(r-1) \frac{a}{2}$, (2.2.4) can be written in the form

$$
\langle f, g\rangle_{\lambda}=\frac{\Gamma_{\Omega}(\lambda)}{(2 \pi)^{2 d}} \int_{\Omega} \frac{d \hat{f}}{d \mu_{\lambda}}(t) \frac{\overline{d \hat{g}}}{d \mu_{\lambda}}(t) N(t)^{\lambda-\frac{d}{r}} d t
$$

(where $\hat{f}, \hat{g}$ are considered as the measures $\hat{f}(t) d t$ and $\hat{g}(t) d t)$. Thus (2.2.18) is the right analogue of (2.2.19), and therefore of (2.2.4). It is an interesting problem to obtain (2.2.18) from (2.2.19) by analytic continuation in the parameter $\lambda$.

\subsection{The case of a symmetric Siegel domain of type $I I$}

Assume now that $e$ is a maximal tripotent in $Z$ which is not unitary. Thus $Z_{1}(e)+Z_{1 / 2}(e)$ and $Z_{1 / 2}(e) \neq 0$. Thus

$$
d_{1}:=\operatorname{dim} Z_{1}(e)=r+r(r-1) \frac{a}{2}, d_{1 / 2}:=\operatorname{dim} Z_{1 / 2}(e)=r b
$$

(where $1 \leq b \in \mathbb{N}) . \quad Z_{1}(e)$ is a $J B^{*}$-algebra which operates on $Z_{1 / 2}(e)$ via

$$
R(z) w=2\{z, e, \eta\}, \quad z \in Z_{1}(e), \quad \eta \in Z_{1 / 2}(e) .
$$

$R: Z_{1}(e) \rightarrow$ End $\left(Z_{1 / 2}(e)\right)$ is a monomorphism of Jordan $*$-algebras, where the involution in End $\left(Z_{1 / 2}(e)\right)$ is induced by the given $K$-invariant inner product $\langle\xi \mid \eta\rangle$ (see [Lo75], Lemma 8.1, p.75). Let us denote

$$
F(\xi, \eta)=\{\xi, \eta, e\}, \quad \xi, \eta \in Z_{1 / 2}(e) .
$$

Then $F: Z_{1 / 2}(e) \times Z_{1 / 2}(e) \rightarrow Z_{1}(e)$ is sesquilinear, and $F(\xi, \xi) \in \bar{\Omega}$ for all $\xi \in Z_{1 / 2}(e)$. We denote also $F(\xi):=F(\xi, \xi)$. Let us define $\tau: Z \times Z \rightarrow Z_{1}(e)$ by

$$
\tau(z, w)=\frac{z_{1}-w_{1}^{*}}{i}-2 F\left(z_{1 / 2}, w_{1 / 2}\right)
$$


where $z=z_{1}+z_{1 / 2}, \quad w=w_{1}+w_{1 / 2} \quad\left(z_{1}, w_{1} \in Z_{1}^{(e)}\right.$ and $\left.z_{1 / 2}, w_{1 / 2} \in Z_{1 / 2}(e)\right)$. For convenience we denote $\tau(z)=\tau(z, z)$. The associated Siegel domain of type II is

$$
T(\Omega):=\{z \in Z ; \quad \tau(z) \in \Omega\} .
$$

It is known that the Cayley transform

$$
c(z)=i \frac{e+z_{1}}{e-z_{1}}+\sqrt{2} R\left(\left(e-z_{1}\right)^{-1}\right)\left(z_{1 / 2}\right), \quad z=z_{1}+z_{1 / 2}
$$

maps the Cartan domain $D$ (i.e. the open unit ball of $Z$ ) biholomorphically onto $T(\Omega)$. Again, for $\lambda \in W(D)$ the operator $V^{(\lambda)} f=\left(f \circ c^{-1}\right)\left(J c^{-1}\right)^{\lambda / p}$ maps $\mathcal{H}_{\lambda}(D)$ isometrically onto $\mathcal{H}_{\lambda}(T(D))$, which is endowed with the inner product (2.2.1). Also, the reproducing kernel of $\mathcal{H}_{\lambda}(T(D))$ is

$$
K^{(\lambda)}(z, w)=N(\tau(z, w))^{-\lambda}, \quad z, w \in T(\Omega) .
$$

Our main goal here is to describe the inner product of $\mathcal{H}_{\lambda}(T(D))$ concretely.

The Shilov boundary of $T(\Omega)$ is the set

$$
H=\{z \in T(\Omega) ; \tau(z)=0\}=\left\{x+i F(\xi)+\xi ; x \in X_{1}(e)+\xi \in Z_{1 / 2}(e)\right\}
$$

Proposition 2.3.1 Let $\xi, \eta \in Z_{1 / 2}(e)$. Then for every $v \in \Omega$

$$
|\langle F(\eta, \xi) \mid v\rangle| \leq\langle F(\xi \mid v)\rangle^{1 / 2}\langle F(\eta \mid v)\rangle^{1 / 2} \leq \frac{1}{2}\langle F(\xi)+F(\eta) \mid v\rangle
$$

Thus

$$
\operatorname{Re} F(\eta, \xi) \leq \frac{1}{2}\langle(F(\xi)+F(\eta)) .
$$

The straightforward proof is based on the positivity of $F$ (i.e. the fact that $F(\xi) \in \bar{\Omega}$ for all $\left.\xi \in Z_{1 / 2}(e)\right)$, and it is omitted.

Corollary 2.3.2 For all $z, w \in T(\Omega)$

$$
\operatorname{Re}(\tau(z, w)) \geq \frac{1}{2}(\tau(z)+\tau(w)) .
$$

In particular $\operatorname{Re}(\tau(z, w)) \in \Omega$, and this is true even if $z \in H$ and $w \in T(\Omega)$.

Proof: Using (2.3.9) we have

$$
\begin{aligned}
2 R \tau(z, w) & =\frac{z_{1}-z_{1}^{*}}{i}+\frac{w_{1}-w_{1}^{*}}{i}-4 \operatorname{Re} F\left(z_{1 / 2}, w_{1 / 2}\right) \\
& \geq \frac{z_{1}-z_{1}^{*}}{i}+\frac{w_{1}-w_{1}^{*}}{i}-2 F\left(z_{1 / 2}\right)-2 F\left(w_{1 / 2}\right)=\tau(z)+\tau(w) .
\end{aligned}
$$

For $\lambda>(r-1) \frac{a}{2}$ consider the measure $d \mu_{\lambda}(x)=N(x)^{\lambda-\frac{d_{1}}{r}} d x$ on $\Omega$. For $\lambda=\ell \frac{a}{2}, 0 \leq \ell \leq r-1$, let $\mu_{\lambda}:=\mu_{\ell}$ be the Lassalle measure (see Theorem 2.2.2 and Section 3.1 below). Then for all $\lambda \in W(D)$

$$
\int_{\Omega} e^{-\langle y \mid x\rangle} d \mu_{\lambda}(x)=\gamma_{\lambda} N(y)^{-\lambda}
$$

with $\gamma_{\lambda}=\Gamma_{\Omega}(\lambda)$ for $\lambda>(r-1) \frac{a}{2}$, and $\gamma_{\lambda}=\gamma_{\ell}=(2 \pi)^{\ell(\ell-1) \frac{a}{2}} \Gamma_{\Omega^{(\ell)}}\left(\ell \frac{a}{2}\right)$ for $\lambda=\ell_{\frac{a}{2}}, 0 \leq \ell \leq r-1$. 
Corollary 2.3.3 Let $z, w \in T(\Omega)$ and let $\lambda \in W(D)$. Then

$$
K^{(\lambda)}(z, w)=\frac{1}{\gamma_{\lambda}} \int_{\Omega} e^{-\langle\tau(z, w) \mid t\rangle} d \mu_{\lambda}(t)
$$

The formula holds also for $z \in H$ and $w \in T(\Omega)$.

Proof: Since $\operatorname{Re} \tau(z, w) \in \Omega$, the integral converges absolutely, and uniformly on compact subsets of $T(\Omega) \times T(\Omega)$. Therefore, the integral is holomorphic in $z$ and anti-holomorphic in $w$. Since $K^{(\lambda)}(z, w)$ is also sesqui-holomorphic, it is enough to show that (2.3.12) holds for $z=w \in T(\Omega)$. Writing $z=x+i y+\xi\left(x \in X,(e), y \in \Omega, \xi \in Z_{1 / 2}(e)\right)$, and using (2.3.11), we obtain

$$
\int_{\Omega} e^{-\langle\tau(z) \mid t\rangle} d \mu_{\lambda}(t)=\gamma_{\lambda} N(\tau(z))^{-\lambda}=\gamma_{\lambda} K^{(\lambda)}(z, z) .
$$

Thus (2.3.12) is established for all $z, w \in T(\Omega)$. Letting $\tau(z) \rightarrow 0$ in (2.3.12) for fixed $w \in T(\Omega)$ (i.e. $z \rightarrow H$ ), we obtain (2.3.12) also for $z \in H$ and $w \in T(\Omega)$.

Lemma 2.3.4 Let $\lambda \in W(D)$, fix $w=u+i v+\eta \in T(\Omega)$ (with $u \in X_{1}(e), v \in \Omega, \quad \eta \in Z_{1 / 2}(e)$ ) and $\xi \in Z_{1 / 2}(e)$, and consider the function

$$
K_{w, \xi}^{(\lambda)}(x)=K^{(\lambda)}(x+i F(\xi)+\xi, w), \quad x \in X_{1}(e) .
$$

Then the Fourier transform of $K_{w, \xi}^{(\lambda)}$, considered as measure, has support in $\bar{\Omega}$, and is given by

$$
\overline{K_{w, \xi}^{(\lambda)}}(t)=\frac{(2 \pi)^{d_{1}}}{\gamma_{\lambda}} \exp \{-\langle F(\xi)+v-2 F(\xi, \eta)+i u \mid t\rangle\} d \mu_{\lambda}(t)
$$

Proof: Using (2.3.12) for $w \in T(\Omega)$ and $z=x+i F(\xi)+\xi \in H$, we obtain

$$
K_{w, \xi}^{(\lambda)}(x)=\frac{1}{\gamma_{\lambda}} \int_{\Omega} e^{i\langle x \mid t\rangle} e^{-\langle F(\xi)+v-2 F(\xi, \eta)+i u \mid t\rangle} d \mu_{\lambda}(t) .
$$

Thus $K_{w, \xi}^{(\lambda)}$ is the inverse Fourier transform of the measure $\gamma_{\lambda}^{-1} \exp \{-\langle F(\xi)+v-2 F(\xi, \eta)+$ $i u|t\rangle\} d \mu_{\lambda}(t)$, whose support is contained in $\bar{\Omega}$. From this (2.3.14) follows by inverting the Fourier transform.

For $\lambda>(r-1) \frac{a}{2}$ we consider on $\Omega \times Z_{1 / 2}(e)$ the measure

$$
d \sigma_{\lambda}(t, \xi)=N(t)^{b} d \mu_{\lambda}(t) d \xi=N(t)^{\lambda-\frac{d_{1}}{r}+b} d t d \xi
$$

Lemma 2.3.5 For every $w \in T(\Omega)$

$$
\iint_{\Omega \times Z_{1 / 2}(e)}\left|\widehat{\frac{K_{w, \xi}^{(\lambda)}}{d \mu_{\lambda}}}(t)\right|^{2} d \sigma_{\lambda}(t, \xi)=\frac{(2 \pi)^{r p}}{\Gamma_{\Omega}(\lambda)} K^{(\lambda)}(w, w) .
$$


Proof: Writing $w=u+i v+\eta$ as in Lemma 2.3.4, we obtain $\mu_{\lambda}$ from (2.3.14)

$$
\begin{aligned}
\int_{Z_{1 / 2}(e)}\left|\frac{d \widehat{K_{w, \xi}^{(\lambda)}}}{d \mu_{\lambda}}(t)\right|^{2} d \xi & =\frac{(2 \pi)^{2 d_{1}}}{\gamma_{\lambda}^{2}} e^{-2\langle v \mid t\rangle} \int_{Z_{1 / 2}(e)} e^{-2\langle F(\xi)-2 \operatorname{Re} F(\xi, \eta) \mid t\rangle} d \xi \\
& =\frac{(2 \pi)^{2 d_{1}}}{\gamma_{\lambda}^{2}} e^{-2\langle v \mid t\rangle} e^{2\langle F(\eta) \mid t\rangle} \int_{Z_{1 / 2}(e)} e^{-2\langle F(\xi-\eta) \mid t\rangle} d \xi \\
& =\frac{(2 \pi)^{2 d_{1}}}{\gamma_{\lambda}^{2}} e^{-\langle\tau(w) \mid t\rangle} \int_{Z_{1 / 2}(e)} e^{-\left\|R\left(t^{1 / 2}\right) \xi\right\|^{2}} d \xi \\
& =\frac{(2 \pi)^{2 d_{1}+r b}}{\gamma_{\lambda}^{2}} N(t)^{-b} e^{-\langle\tau(w) \mid t\rangle} .
\end{aligned}
$$

Here we used the well-known formula

$$
\langle x \mid\{y, z, w\}\rangle=\langle\{x, y, z\} \mid w\rangle, \quad \forall x, y, z, w \in Z
$$

to obtain

$$
\left\|R\left(t^{1 / 2}\right) \xi\right\|^{2}=\langle\xi \mid R(t) \xi\rangle=\langle\xi \mid 2\{t, e, \xi\}\rangle=2\langle\{\xi, \xi, e\} \mid t\rangle=2\langle F(\xi) \mid t\rangle .
$$

It follows that

$$
\begin{aligned}
& \iint_{\Omega \times Z_{1 / 2}(e)}\left|\frac{d \widehat{K_{w, \xi}^{(\lambda)}}}{d \mu_{\lambda}}(t)\right|^{2} d \xi N(t)^{b} d \mu_{\lambda}(t)= \\
& =\frac{(2 \pi)^{r p}}{\gamma_{\lambda}^{2}} \int_{\Omega} e^{-\langle\tau(w) \mid t\rangle} d \mu_{\lambda}(t) \\
& =\frac{(2 \pi)^{r p}}{\Gamma_{\Omega}(\lambda)} \cdot N(\tau(w))^{-\lambda}=\frac{(2 \pi)^{r p}}{\Gamma_{\Omega}(\lambda)} K^{(\lambda)}(w, w) .
\end{aligned}
$$

Corollary 2.3.6 Let $\lambda>(r-1) \frac{a}{2}$. For all $z, w \in T(\Omega)$

$$
\int_{\Omega} \int_{Z_{\frac{1}{2}}(e)} \frac{\widehat{d K_{w, \xi}^{(\lambda)}}}{d t)} \frac{\widehat{d \bar{K}_{z, \xi}^{(\lambda)}}}{d \nu_{\lambda}}(t) d \sigma_{\lambda}(t, \xi)=\frac{(2 \pi)^{r p}}{\Gamma_{\Omega}(\lambda)} K^{(\lambda)}(z, w) .
$$

Also, considering $\widehat{K_{w, \xi}^{(\lambda)}}(t)$ as a function, we have

$$
\int_{\Omega} \int_{Z_{\frac{1}{2}}(e)} \widehat{K_{w, \xi}^{(\lambda)}}(t) \widehat{K_{z, \xi}^{(\lambda)}}(t) d \xi N(t)^{\frac{d}{r}-\lambda} d t=\frac{(2 \pi)^{r p}}{\Gamma_{\Omega}(\lambda)} K^{(\lambda)}(z, w) .
$$

Proof: Both sides of (2.3.18) are sesqui-holomorphic in $(z, w)$ and coincide on the "diagonal" $z=w$ by Lemma 2.3.5. Hence they coincide for all $z, w \in T(\Omega)$. (2.3.19) is an obvious consequence of (2.3.18), since

$$
\frac{\widehat{d K_{w, \xi}^{(\lambda)}}}{d \nu_{\lambda}}(t) \cdot N(t)^{\lambda-\frac{d}{r}}=\widehat{K_{w, \xi}^{(\lambda)}}(t) .
$$

The generalization of Proposition 2.2.1 to Siegel domains of type II is the following result. 
Theorem 2.3.7 Let $T(\Omega)$ be a symmetric Siegel domain of type II, let $\lambda>(r-1) \frac{a}{2}$, and let $f$ be a holomorphic function on $T(\Omega)$. Then the following conditions are equivalent

(i) $f \in \mathcal{H}_{\lambda}(T(\Omega))$;

(ii) The boundary values of $f$ at points $z=x+i F(\xi)+\xi\left(x \in X_{1}(e), \xi \in Z_{\frac{1}{2}}(e)\right)$ of the Shilov boundary $H$, i.e.

$$
f_{\xi}(x)=f(x+i F(\xi)+\xi)=\lim _{\Omega \ni y \rightarrow 0} f(x+i y+i F(\xi)+\xi)
$$

exist almost everywhere, the Fourier transform $\hat{f}_{\xi}(t)$ is supported in $\bar{\Omega}$, and

$$
\int_{\Omega} \int_{Z_{\frac{1}{2}}(e)}\left|\hat{f}_{\xi}(t)\right|^{2} d \xi N(t)^{\frac{d}{r}-\lambda} d t<\infty
$$

Moreover, the operator $V_{\lambda}: \mathcal{H}_{\lambda}(T(\Omega)) \rightarrow L^{2}\left(\Omega \times Z_{\frac{1}{2}}(e), N(t)^{\frac{d}{r}-\lambda} d t d \xi\right)$ defined by

$$
\left(V_{\lambda} f\right)(t, \xi)=\frac{\Gamma_{\Omega}(\lambda)^{\frac{1}{2}}}{(2 \pi)^{\frac{r p}{2}}} \hat{f}_{\xi}(t)
$$

is an isometry of $\mathcal{H}_{\lambda}(T(\Omega))$ onto $L^{2}\left(\Omega \times Z_{\frac{1}{2}}(e), N(t)^{\frac{d}{r}-\lambda} d t d \xi\right)$. In particular, for every $f, g \in$ $\mathcal{H}_{\lambda}(T(\Omega))$

$$
<f, g>_{\lambda}=\frac{\Gamma_{\Omega}(\lambda)}{(2 \pi)^{r p}} \int_{\Omega} \int_{Z_{\frac{1}{2}}(e)} \hat{f}_{\xi}(t) \overline{\hat{g}_{\xi}(t)} d \xi N(t)^{\frac{d}{r}-\lambda} d t .
$$

The proof uses (2.3.19) and is analogous to the proofs of Proposition 2.2.1 (i.e. Proposition 6.1 and Theorem 6.1 of [AU97]) and to the proof of Theorem 2.2.7. Therefore we omit it. We remark that in view of $(2.3 .18),(2.3 .22)$ can be written in the form

$$
<f, g>_{\lambda}=\frac{\Gamma_{\Omega}(\lambda)}{(2 \pi)^{r p}} \int_{\Omega} \int_{Z_{\frac{1}{2}}(e)} \frac{d \hat{f}_{\xi}(t)}{d \nu_{\lambda}} \frac{\overline{d \hat{g}_{\xi}(t)}}{d \nu_{\lambda}} d \sigma_{\lambda}(t, \xi),
$$

where $\sigma_{\lambda}$ is the measure defined by (2.3.15).

We turn now to the case where $\lambda=\ell_{\frac{a}{2}}, 0 \leq \ell \leq r-1$ (and for simplicity denote $\nu_{\ell \frac{a}{2}}=\nu_{\ell}$ and $\gamma_{\ell \frac{a}{2}}=\gamma_{\ell}$. Let $t \in \partial_{\ell} \Omega$, then its support idempotent $s(t)$ has rank $\ell$. Thus $Z_{\frac{1}{2}}(e)$ is the direct sum

$$
Z_{\frac{1}{2}}(e)=\left(Z_{\frac{1}{2}}(e) \cap Z_{\frac{1}{2}}(s(t))\right)+\left(Z_{\frac{1}{2}}(e) \cap Z_{0}(s(t))\right) .
$$

Let us denote

$$
\partial_{\ell}(\widehat{T(\Omega)})=\left\{t+\xi ; t \in \partial_{\ell} \Omega, \xi \in Z_{\frac{1}{2}}(e) \cap Z_{\frac{1}{2}}(s(t))\right\} .
$$

(The notation is chosen as to indicate that the Fourier transforms of functions in $\mathcal{H}_{\ell \frac{a}{2}}(T(\Omega)$ ) are supported in $\left.\partial_{\ell}(\widehat{T(\Omega)})\right) . \quad \partial_{\ell}(\widehat{T(\Omega)})$ can be viewed as a bundle whose base is $\partial_{\ell} \Omega$, and the fiber over $t \in \partial_{\ell} \Omega$ is $Z_{\frac{1}{2}}(e) \cap Z_{\frac{1}{2}}(s(t))$. Let us consider on $\partial_{\ell}(\widehat{T(\Omega)})$ the measure $\tilde{\mu}_{\ell}$, defined by

$$
\int_{\partial_{\ell}(\widehat{T(\Omega)})} f d \tilde{\mu}_{\ell}=\int_{\partial_{\ell} \Omega}\left(\int_{Z_{\frac{1}{2}}(e) \cap Z_{\frac{1}{2}}(s(t))} f(t+\xi) d \xi\right) d \mu_{\ell}(t) .
$$


For every $t \in \partial_{\ell} \Omega$ let $\operatorname{det}(t)=N_{X_{1}(s(t))}(t)$ be the determinant of $t$ in the Jordan algebra $X_{1}(s(t))$. We define a measure $\sigma_{\ell}$ on $\partial_{\ell}(\widehat{T(\Omega)})$ via

$$
\int_{\partial_{\ell}(\widehat{T(\Omega)})} f d \sigma_{\ell}=\int_{\partial_{\ell} \Omega}\left(\int_{Z_{\frac{1}{2}}(e) \cap Z_{\frac{1}{2}}(s(t))} f(t+\xi) d \xi\right) \operatorname{det}(t)^{b} d \mu_{\ell}(t),
$$

i.e., $d \sigma_{\ell}(t, \xi)=\operatorname{det}(t)^{b} d \tilde{\mu}_{\ell}(t, \xi)$. Namely, on the base $\partial_{\ell} \Omega$ we use the measure $\mu_{\ell}$ and at the fiber above $t \in \partial_{\ell} \Omega$ we use the measure $\operatorname{det}(t)^{b} d \xi$. Notice the analogy between $\sigma_{\ell}$ and $\sigma_{\lambda}$ for $\lambda>(r-1) \frac{a}{2}$.

Lemma 2.3.8 Let $0 \leq \ell \leq r-1$ and fix $w=u+i v+\eta \in T(\Omega)$ (where $u \in X_{1}(e), v \in \Omega$ and $\eta \in Z_{\frac{1}{2}}(e)$ ). Then the Fourier transform (with respect to $x$ ) of $K_{w, \xi}^{\left(\ell^{\frac{a}{2}}\right)}(x)=K_{w}^{\left(\ell \frac{a}{2}\right)}(x+i F(\xi)+\xi)$ is a measure on $\partial_{\ell}(\widehat{T(\Omega)})$ which is absolutely continuous with respect to $\tilde{\mu}_{\ell}$, and

$$
\frac{d K_{w}^{\left(\ell \frac{a}{2}\right)}}{d \tilde{\mu}_{\ell}}(t, \xi)=\frac{(2 \pi)^{d_{1}}}{\gamma_{\ell}} \exp \left(-\left\langle i u-2 i \Im F(\xi, \eta)+\frac{1}{2} \tau(w)+F(\xi-\eta) \mid t\right\rangle\right) .
$$

Moreover, with $\chi_{\ell}=(2 \pi)^{2 d_{1}+\ell b-\ell(r-\ell) \frac{a}{2}} \cdot 2^{-\ell b}$ we have

$$
\int_{\partial_{\ell}(\widehat{T(\Omega)})}\left|\frac{d K_{w}^{\left(\ell \frac{a}{2}\right)}}{d \tilde{\mu}_{\ell}}\right|^{2} d \sigma_{\ell}=\frac{\chi_{\ell}}{\gamma_{\ell}} K^{\left(\ell \frac{a}{2}\right)}(w, w) .
$$

Proof: Using Lemma 2.3.4 for $\lambda=\ell \frac{a}{2}$, we see that for $t \in \partial_{\ell} \Omega$ and $\xi \in Z_{\frac{1}{2}}(e)$,

$$
\widehat{K_{w, \xi}^{\left(\ell \frac{a}{2}\right)}}(t)=\frac{(2 \pi)^{d_{1}}}{\gamma_{\ell}} \exp (-\langle i u+v+F(\xi)-2 F(\xi, \eta) \mid t\rangle) d \mu_{\ell}(t) .
$$

It is easy to see that for all $\xi, \eta \in Z_{\frac{1}{2}}(e)$

$$
\langle F(\xi, \eta) \mid t\rangle=\left\langle F\left(P_{\frac{1}{2}}(s(t)) \xi, P_{\frac{1}{2}}(s(t)) \eta\right) \mid t\right\rangle .
$$

Hence, the measure $\widehat{K_{w}^{\left(\ell \frac{a}{2}\right)}}$ is supported in $\partial_{\ell}(\widehat{T(\Omega)})$, it is absolutely continuous with respect to $\tilde{\mu}_{\ell}$, and its Radon-Nikodym derivative with respect to $\tilde{\mu}_{\ell}$ is given by (2.3.28). Next, using (2.3.30) we see that for fixed $t \in \partial_{\ell} \Omega$

$$
\begin{aligned}
\int_{Z_{\frac{1}{2}}(e) \cap Z_{\frac{1}{2}}(s(t))}\left|\frac{d K_{w}^{\left(\ell \frac{a}{2}\right)}}{d \tilde{\mu}_{\ell}}(t, \xi)\right|^{2} d \xi & =\frac{(2 \pi)^{2 d_{1}}}{\gamma_{\ell}^{2}} e^{-\langle\tau(w) \mid t\rangle} \int_{Z_{\frac{1}{2}}(e) \cap Z_{\frac{1}{2}}(s(t))} e^{-2\langle F(\xi) \mid t\rangle} d \xi \\
& =\frac{(2 \pi)^{2 d_{1}} \pi^{\ell b}}{\gamma_{\ell}^{2}} e^{-\langle\tau(w) \mid t\rangle} \operatorname{det}(t)^{-b} .
\end{aligned}
$$

Hence, using Corollary 2.3.3, we obtain

$$
\begin{aligned}
\int_{\partial_{\ell}(\widehat{T(\Omega)})}\left|\frac{d \widehat{K_{w}^{\left(\ell \frac{a}{2}\right)}}}{d \tilde{\mu}_{\ell}}(t, \xi)\right|^{2} d \sigma_{\ell}(t, \xi) & =\frac{(2 \pi)^{2 d_{1}} \pi^{\ell b}}{\gamma_{\ell}^{2}} \int_{\partial_{\ell} \Omega} e^{-\langle\tau(w) \mid t\rangle} d \mu_{\ell}(t) \\
& =\frac{\chi_{\ell}}{\Gamma_{\Omega^{(\ell)}\left(\ell \frac{a}{2}\right)}} N(\tau(w))^{-\ell \frac{a}{2}}=\frac{\chi_{\ell}}{\Gamma_{\Omega^{(\ell)}}\left(\ell \frac{a}{2}\right)} K^{\left(\ell \frac{a}{2}\right)}(w, w) .
\end{aligned}
$$


Corollary 2.3.9 Let $0 \leq \ell \leq r-1$. For every $z, w \in T(\Omega)$,

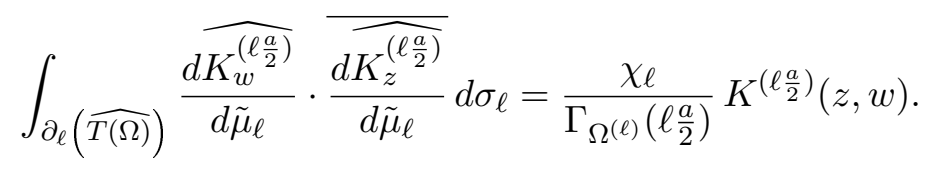

Proof: Both sides of (2.3.31) are holomorphic in $z$ and anti-holomorphic in $w$, and they coincide on the "diagonal" $z=w$. Hence they coincide for all $z, w \in T(\Omega)$.

Theorem 2.3.10 Let $T(\Omega)$ be a symmetric Siegel domain of type II, let $0 \leq \ell \leq r-1$, and let $f$ be a holomorphic function on $T(\Omega)$. The following conditions are equivalent

(i) $f \in \mathcal{H}_{\ell \frac{a}{2}}(T(\Omega))$;

(ii) The boundary values of $f$

$$
f_{\xi}(x)=f(x+i F(\xi)+\xi)=\lim _{\Omega \ni y \rightarrow 0} f(x+i y+i F(\xi)+\xi)
$$

exist for almost all points $x+i F(\xi)+\xi$ of the Shilov boundary $H$, the Fourier transform $\hat{f}_{\xi}(t):=\int_{X_{1}(e)} e^{-i\langle x \mid t\rangle} f_{\xi}(x) d x$ is a measure with support in $\partial_{\ell}(\widehat{T(\Omega)})$ which is absolutely continuous with respect to $\tilde{\mu}_{\ell}$, and the Radon-Nikodym derivative $\frac{\partial \hat{f}}{\partial \tilde{\mu}_{\ell}}$ belongs to $L^{2}\left(\partial_{\ell}(\widehat{T(\Omega)}), \sigma_{\ell}\right)$.

Moreover, the operator $V_{\ell}: \mathcal{H}_{\ell \frac{a}{2}}(T(\Omega)) \rightarrow L^{2}\left(\partial_{\ell}(\widehat{T(\Omega)}), \sigma_{\ell}\right)$ defined via

$$
\left(V_{\ell} f\right)(t, \xi)=\left(\frac{\Gamma_{\Omega^{(\ell)}}\left(\ell \frac{a}{2}\right)}{\chi_{\ell}}\right)^{\frac{1}{2}} \frac{\partial \hat{f}}{\partial \tilde{\mu}_{\ell}}(t, \xi)
$$

is a surjective isometry. Thus, for all $f, g \in \mathcal{H}_{\ell \frac{a}{2}}(T(\Omega))$,

$$
\begin{aligned}
<f, g>_{\ell^{a}} & =\frac{\Gamma_{\Omega^{(\ell)}}\left(\ell \frac{a}{2}\right)}{\chi_{\ell}} \int_{\partial_{\ell}(\widehat{T(\Omega)})} \frac{\partial \hat{f}}{\partial \tilde{\mu}_{\ell}} \cdot \frac{\overline{\partial \hat{g}}}{\partial \tilde{\mu}_{\ell}} d \sigma_{\ell} \\
& =\frac{\Gamma_{\Omega^{(\ell)}\left(\ell \frac{a}{2}\right)}}{\chi_{\ell}} \int_{\partial_{\ell} \Omega}\left(\int_{Z_{\frac{1}{2}}(e) \cap Z_{\frac{1}{2}}(s(t))} \frac{\partial \hat{f}}{\partial \tilde{\mu}_{\ell}}(t, \xi) \cdot \overline{\frac{\partial \hat{g}}{\partial \tilde{\mu}_{\ell}}(t, \xi)} \operatorname{det}(t)^{b} d \xi\right) d \nu_{\ell}(t) .
\end{aligned}
$$

The proof of Theorem 2.3.10 uses Lemma 2.3.8 and Corollary 2.3.9, as well as the standard arguments used in the proofs of Proposition 2.2.1 and Theorem 2.2.7; it is therefore omitted.

Although the bundle $\partial_{\ell}(\widehat{T(\Omega)})$ and the measure $\sigma_{\ell}$ give natural and canonical description of the space $\mathcal{H}_{\ell \frac{a}{2}}$ and its inner product (Theorem 2.3.10), they are not easy to use in some concrete computation. We therefore develop now a formula for $\left\langle f, g>_{\ell \frac{a}{2}}\right.$ analogous to (2.3.33) with more concrete space and measure, which are however not invariant.

Recall that $u_{\ell}=\sum_{j=1}^{\ell} e_{j}, v_{\ell}=\sum_{j=\ell+1}^{r} e_{j}$. We write

$$
Z_{\frac{1}{2}}^{\left(\frac{1}{2}\right)}=Z_{\frac{1}{2}}(e) \cap Z_{\frac{1}{2}}\left(u_{\ell}\right), \quad Z_{\frac{1}{2}}^{(0)}=Z_{\frac{1}{2}}(e) \cap Z_{0}\left(u_{\ell}\right) .
$$


Thus $Z_{\frac{1}{2}}(e)=Z_{\frac{1}{2}}^{\left(\frac{1}{2}\right)}+Z_{\frac{1}{2}}^{(0)}$. Recall also (see Lemma 2.2.3) that every $t \in N_{\Omega} A\left(u_{\ell}\right) \subset \partial_{\ell} \Omega$ has Peirce decomposition $t=t_{1}+t_{\frac{1}{2}}+t_{0}$, where $t_{1} \in X_{1}\left(u_{\ell}\right)$ positive and invertible, $t_{\frac{1}{2}} \in X_{\frac{1}{2}}\left(u_{\ell}\right)=$ $X_{\frac{1}{2}}\left(v_{\ell}\right)$, and $t_{0} \in X_{1}\left(v_{\ell}\right)$ depends on $t_{1}$ and $t_{\frac{1}{2}}$ via

$$
t_{0}=2 v_{\ell}\left(t_{\frac{1}{2}}\left(t_{\frac{1}{2}} t_{1}^{-1}\right)\right)
$$

where $t_{1}^{-1}$ is the inverse of $t_{1}$ in $X_{1}\left(u_{\ell}\right)$.

Lemma 2.3.11 For every $\xi=\xi_{\frac{1}{2}}+\xi_{0} \in Z_{\frac{1}{2}}$ (with $\xi_{\frac{1}{2}} \in Z_{\frac{1}{2}}^{\left(\frac{1}{2}\right)}$ and $\xi_{0} \in Z_{\frac{1}{2}}^{(0)}$ ) and every $t=t_{1}+t_{\frac{1}{2}}+t_{0} \in N_{\Omega} A\left(u_{\ell}\right)$,

$$
2\langle F(\xi) \mid t\rangle=\left\|R\left(t_{1}^{\frac{1}{2}}\right) \xi_{\frac{1}{2}}+R\left(t_{1}^{-\frac{1}{2}}\right) R\left(t_{\frac{1}{2}}\right) \xi_{0}\right\|^{2}
$$

where $t_{1}^{-\frac{1}{2}}$ is the inverse of $t_{1}^{\frac{1}{2}}$ in $X_{1}\left(u_{\ell}\right)$.

Proof: (2.3.36) will follow as soon as we prove that

$$
\begin{aligned}
& 2\left\langle\left\{\xi_{\frac{1}{2}}, \xi_{\frac{1}{2}}, u_{\ell}\right\} \mid t_{1}\right\rangle=\left\|R\left(t_{1}^{\frac{1}{2}}\right) \xi_{\frac{1}{2}}\right\|^{2} \\
& 2\left\langle\left\{\xi_{0}, \xi_{\frac{1}{2}}, u_{\ell}\right\} \mid t_{\frac{1}{2}}\right\rangle=\left\langle R\left(t_{1}^{-\frac{1}{2}}\right) R\left(t_{\frac{1}{2}}\right) \xi_{0} \mid R\left(t_{1}^{\frac{1}{2}}\right) \xi_{\frac{1}{2}}\right\rangle \\
& 2\left\langle\left\{\xi_{\frac{1}{2}}, \xi_{0}, v_{\ell}\right\} \mid t_{\frac{1}{2}}\right\rangle=\left\langle R\left(t_{1}^{\frac{1}{2}}\right) \xi_{\frac{1}{2}} \mid R\left(t_{1}^{-\frac{1}{2}}\right) R\left(t_{\frac{1}{2}}\right) \xi_{0}\right\rangle
\end{aligned}
$$

and

$$
2\left\langle\left\{\xi_{0}, \xi_{0}, v_{\ell}\right\} \mid t_{0}\right\rangle=\left\|R\left(t_{1}^{-\frac{1}{2}}\right) R\left(t_{\frac{1}{2}}\right) \xi_{0}\right\|^{2} .
$$

Indeed, by the "Peirce calculus" and orthogonality of the Peirce spaces

$$
\begin{aligned}
2\langle F(\xi) \mid t\rangle= & 2\left\langle\left\{\xi_{\frac{1}{2}}+\xi_{0}, \xi_{\frac{1}{2}}+\xi_{0}, u_{\ell}+v_{\ell}\right\} \mid t_{1}+t_{\frac{1}{2}}+t_{0}\right\rangle \\
= & 2\left\langle\left\{\xi_{\frac{1}{2}}, \xi_{\frac{1}{2}}, u_{\ell}\right\} \mid t_{1}\right\rangle+2\left\langle\left\{\xi_{0}, \xi_{0}, v_{\ell}\right\} \mid t_{0}\right\rangle \\
& +2\left\langle\left\{\xi_{0}, \xi_{\frac{1}{2}}, u_{\ell}\right\} \mid t_{\frac{1}{2}}\right\rangle+2\left\langle\left\{\xi_{\frac{1}{2}}, \xi_{0}, v_{\ell}\right\} \mid t_{\frac{1}{2}}\right\rangle .
\end{aligned}
$$

Using the fact that $R: Z_{1}(e) \rightarrow \operatorname{End}\left(Z_{\frac{1}{2}}(e)\right)$ is a monomorphism of Jordan-*-algebras, (see [Lo75], Lemma 8.1, p. 75), we see that $\left.R\right|_{Z_{1}\left(u_{\ell}\right)}: Z_{1}\left(u_{\ell}\right) \rightarrow Z_{\frac{1}{2}}^{\left(\frac{1}{2}\right)}$ is also a monomorphism of Jordan-*-algebras. In particular, for every $\xi_{\frac{1}{2}} \in Z_{\frac{1}{2}}^{\left(\frac{1}{2}\right)}$,

$$
R\left(t_{1}^{\frac{1}{2}}\right) R\left(t_{1}^{\frac{1}{2}}\right) \xi_{\frac{1}{2}}=R\left(t_{1}\right) \xi_{\frac{1}{2}} \quad \text { and } \quad R\left(t_{1}^{-\frac{1}{2}}\right) R\left(t_{1}^{\frac{1}{2}}\right) \xi_{\frac{1}{2}}=R\left(u_{\ell}\right) \xi_{\frac{1}{2}}=\xi_{\frac{1}{2}}
$$

It follows that

$$
\begin{aligned}
\left\|R\left(t_{1}^{\frac{1}{2}}\right) \xi_{\frac{1}{2}}\right\|^{2} & =\left\langle\xi_{\frac{1}{2}} \mid R\left(t_{1}^{\frac{1}{2}}\right)^{*} R\left(t_{1}^{\frac{1}{2}}\right) \xi_{\frac{1}{2}}\right\rangle=\left\langle\xi_{\frac{1}{2}} \mid R\left(t_{1}\right) \xi_{\frac{1}{2}}\right\rangle \\
& =2\left\langle\xi_{\frac{1}{2}} \mid\left\{\xi_{\frac{1}{2}}, u_{\ell}, t_{1}\right\}\right\rangle, \text { since } t_{1} \text { is orthogonal to } v_{\ell} \\
& =2\left\langle\left\{\xi_{\frac{1}{2}}, \xi_{\frac{1}{2}}, u_{\ell}\right\} \mid t_{1}\right\rangle, \text { by }(2.3 .17),
\end{aligned}
$$


and (2.3.37) is established. Using similar arguments and the fact that $R\left(t_{\frac{1}{2}}\right) Z_{\frac{1}{2}}^{(0)} \subset Z_{\frac{1}{2}}^{\left(\frac{1}{2}\right)}$, we obtain

$$
\begin{aligned}
\left\langle R\left(t_{1}^{\frac{1}{2}}\right) \xi_{\frac{1}{2}} \mid R\left(t_{1}^{-\frac{1}{2}}\right) R\left(t_{\frac{1}{2}}\right) \xi_{0}\right\rangle & =\left\langle\xi_{\frac{1}{2}} \mid R\left(t_{1}^{\frac{1}{2}}\right)^{*} R\left(t_{1}^{-\frac{1}{2}}\right) R\left(t_{\frac{1}{2}}\right) \xi_{0}\right\rangle=\left\langle\xi_{\frac{1}{2}} \mid R\left(t_{\frac{1}{2}}\right) \xi_{0}\right\rangle \\
& =2\left\langle\xi_{\frac{1}{2}} \mid\left\{\xi_{0}, v_{\ell}, t_{\frac{1}{2}}\right\}\right\rangle, \text { since } \xi_{0} \text { is orthogonal to } u_{\ell} \\
& =2\left\langle\left\{\xi_{\frac{1}{2}}, \xi_{0}, v_{\ell}\right\} \mid t_{\frac{1}{2}}\right\rangle .
\end{aligned}
$$

This establishes (2.3.39). The proof of (2.3.38) is similar and is therefore omitted. To prove (2.3.40), notice first that

$$
\begin{aligned}
\left\|R\left(t_{1}^{-\frac{1}{2}}\right) R\left(t_{\frac{1}{2}}\right) \xi_{0}\right\|^{2} & =\left\langle\xi_{0} \mid R\left(t_{\frac{1}{2}}\right) R\left(t_{1}^{-\frac{1}{2}}\right)^{*} R\left(t_{1}^{-\frac{1}{2}}\right) R\left(t_{\frac{1}{2}}\right) \xi_{0}\right\rangle \\
& =\left\langle\xi_{0} \mid R\left(t_{\frac{1}{2}}\right) R\left(t_{1}^{-1}\right) R\left(t_{\frac{1}{2}}\right) \xi_{0}\right\rangle .
\end{aligned}
$$

Next, since $R$ is a Jordan homomorphism, it preserves the "quadratic representation" operator $P(x):=2 M(x)^{2}-M\left(x^{2}\right)$ (where $M(x) y:=x y=\{x, e, y\} \quad \forall x, y \in Z_{1}(e)$ ). Thus

$$
\begin{aligned}
R\left(t_{\frac{1}{2}}\right) R\left(t_{1}^{-1}\right) R\left(t_{\frac{1}{2}}\right) & =R\left(P\left(t_{\frac{1}{2}}\right) t_{1}^{-1}\right) \\
& =2 R\left(M\left(t_{\frac{1}{2}}\right)^{2} t_{1}^{-1}\right)-R\left(M\left(t_{\frac{1}{2}}^{2}\right) t_{1}^{-1}\right) .
\end{aligned}
$$

Now, $M\left(t_{\frac{1}{2}}\right)^{2} t_{1}^{-1}=t_{\frac{1}{2}}\left(t_{\frac{1}{2}} t_{1}^{-1}\right) \in X_{1}\left(u_{\ell}\right)+X_{1}\left(v_{\ell}\right)$, hence

$$
\begin{aligned}
2 R\left(M\left(t_{\frac{1}{2}}\right)^{2} t_{1}^{-1}\right) \xi_{0} & =4\left\{t_{\frac{1}{2}}\left(t_{\frac{1}{2}} t_{1}^{-1}\right), v_{\ell}, \xi_{0}\right\}, \text { since } \xi_{0} \text { is orthogonal to } u_{\ell} \\
& =4\left\{v_{\ell}\left(t_{\frac{1}{2}}\left(t_{\frac{1}{2}} t_{1}^{-1}\right)\right), v_{\ell}, \xi_{0}\right\} \\
& =2\left\{t_{0}, v_{\ell}, \xi_{0}\right\}, \text { by }(2.3 .35) .
\end{aligned}
$$

Next, $t_{\frac{1}{2}}^{2} \in X_{1}\left(u_{\ell}\right)+X_{1}\left(v_{\ell}\right)$. Hence $M\left(t_{\frac{1}{2}}^{2}\right) t_{1}^{-1} \in X_{1}\left(u_{\ell}\right)$, and therefore $R\left(M\left(t_{\frac{1}{2}}^{2}\right) t_{1}^{-1}\right) \xi_{0}=0$. It follows that

$$
\begin{aligned}
\left\|R\left(t_{1}^{-\frac{1}{2}}\right) R\left(t_{\frac{1}{2}}\right) \xi_{0}\right\|^{2} & =\left\langle\xi_{0} \mid 2 R\left(M\left(t_{\frac{1}{2}}\right)^{2} t_{1}^{-1}\right) \xi_{0}-R\left(M\left(t_{\frac{1}{2}}^{2}\right) t_{1}^{-1}\right) \xi_{0}\right\rangle \\
& =2\left\langle\xi_{0} \mid\left\{\xi_{0}, v_{\ell}, t_{0}\right\}\right\rangle=2\left\langle\left\{\xi_{0}, \xi_{0}, v_{\ell}\right\} \mid t_{0}\right\rangle
\end{aligned}
$$

and (2.3.40) is established. This completes the proof of Lemma 2.3.11.

Let us define a measure $\tilde{\sigma}_{\ell}$ on the set $N_{\Omega} A\left(u_{\ell}\right) \times Z_{\frac{1}{2}}^{\left(\frac{1}{2}\right)}$ via

$$
\int_{N_{\Omega} A\left(u_{\ell}\right)} \int_{Z_{\frac{1}{2}}^{\left(\frac{1}{2}\right)}} f d \tilde{\sigma}_{\ell}=\int_{N_{\Omega} A\left(u_{\ell}\right)}\left(\int_{Z_{\frac{1}{2}}^{\left(\frac{1}{2}\right)}} f\left(t+\xi_{\frac{1}{2}}\right) d \xi_{\frac{1}{2}}\right) N_{\ell}\left(t_{1}\right)^{b} d \mu_{\ell}(t) .
$$

Notice the analogy between $\tilde{\sigma}_{\ell}$ and $\sigma_{\ell}$ (and the fact that they use the same number of variables. The advantage of $\tilde{\sigma}_{\ell}$ is that it uses fixed coordinates $\left(t_{1}, t_{1 / 2}, \xi_{1 / 2}\right) \in \Omega_{1}\left(u_{\ell}\right) \times X_{1 / 2}\left(u_{\ell}\right) \times Z_{1 / 2}^{(1 / 2)}$.

Lemma 2.3.12 Let $0 \leq \ell \leq r-1$ and $w \in T(\Omega)$. Then

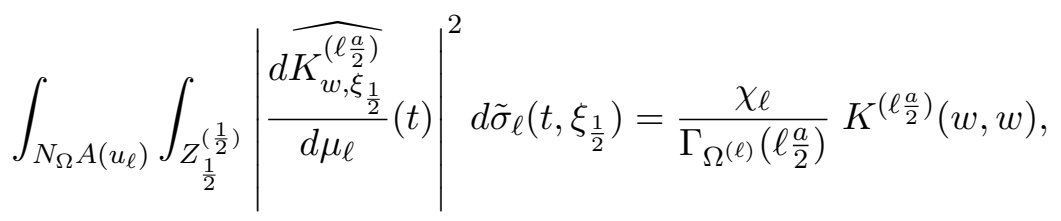

where $\chi_{\ell}$ is as in Lemma 2.3.8. 
Proof: Write $w=u+i v+\eta$ with $u \in X_{1}(e), v \in \Omega$ and $\eta \in Z_{\frac{1}{2}}$, and fix $t \in N_{\Omega} A\left(u_{\ell}\right)$ with Peirce decomposition $t=t_{1}+t_{\frac{1}{2}}+t_{0}$ with $t_{\alpha} \in X_{\alpha}\left(u_{\ell}\right)$ and $t_{0}$ given by (2.3.35). Then

$$
\left|\widehat{d K_{w, \xi_{\frac{1}{2}}}^{\left(\ell \frac{a}{2}\right)}}(t)\right|^{2}=\frac{(2 \pi)^{2 d_{1}}}{\gamma_{\ell}^{2}} e^{-\langle\tau(w) \mid t\rangle} e^{-2\left\langle F\left(\xi_{\frac{1}{2}}-\eta\right) \mid t\right\rangle}
$$

and in view of Lemma 2.3.11,

$$
\begin{aligned}
\int_{Z_{1 / 2}^{(1 / 2)}} e^{-2\left\langle F\left(\xi_{1 / 2}-\eta\right) \mid t\right\rangle} d \xi_{1 / 2} & =\int_{Z_{1 / 2}^{(1 / 2)}} e^{-\left\|R\left(t_{1}^{1 / 2}\right)\left(\xi_{1 / 2}-\eta_{1 / 2}\right)-R\left(t_{1}^{-1 / 2}\right) R\left(t_{1 / 2}\right) \eta_{0}\right\|^{2}} d \xi_{1 / 2} \\
& =\int_{Z_{1 / 2)}^{(1 / 2)}} e^{-\left\|z_{1 / 2}\right\|^{2}} d\left(R\left(t_{1}^{-1 / 2}\right) z_{1 / 2}\right)=\pi^{\ell b} N_{\ell}\left(t_{1}\right)^{-b} .
\end{aligned}
$$

Using this and the knowledge of the Laplace transform of $\mu_{\ell}$ (see Theorem 2.2.2), we obtain

$$
\begin{aligned}
& \int_{N_{\ell} A\left(u_{\ell}\right)} \int_{Z_{1 / 2}^{(1 / 2)}}\left|\frac{d \widehat{K_{w, \frac{a}{\left.\xi_{1 / 2}\right)}}^{\left(\xi_{1}\right)}}}{d \mu_{\ell}}(t)\right|^{2} d \xi_{1 / 2} N_{\ell}\left(t_{1}\right)^{b} d \mu_{\ell}(t)= \\
& =\frac{(2 \pi)^{2 d_{1}+\ell b}}{\gamma_{\ell}^{2}} \int_{N_{\Omega} A\left(u_{\ell}\right)} e^{-\langle\tau(w) \mid t\rangle} d \mu_{\ell}(t) \\
& =\frac{(2 \pi)^{2 d_{1}+\ell b}}{\gamma_{\ell} 2^{\ell b}} N(\tau(w))^{-\ell \frac{a}{2}}=\frac{\chi_{\ell}}{\Gamma_{\Omega^{(\ell)}}\left(\ell \frac{a}{2}\right)} K^{\left(\ell \frac{a}{2}\right)}(w, w) .
\end{aligned}
$$

Theorem 2.3.13 Let $T(\Omega)$ be a symmetric Siegel domain of type II. Let $0 \leq \ell \leq r-1$, and let $f$ be a holomorphic function on $T(\Omega)$. Then the following conditions are equivalent:

(i) $f \in \mathcal{H}_{\ell \frac{a}{2}}(T(\Omega))$;

(ii) The boundary values of $f$ at points of the Shilov boundary $H$ :

$$
f_{\xi}(x):=f(x+i F(\xi)+\xi) \lim _{\Omega \ni y \rightarrow 0} f(x+i y+i F(\xi)+\xi)
$$

exist almost everywhere on $H$, the Fourier transform $\hat{f}_{\xi}(t)$ is a measure with support in $\partial_{\ell} \Omega$ which is absolutely continuous with respect to $\mu_{\ell}$, and the Radon-Nikodym derivative $\frac{d \hat{f}_{\xi}}{d \mu_{\ell}}(t)$ satisfies

$$
\int_{\partial_{\ell}(\Omega)} \int_{Z_{1 / 2}^{(1 / 2)}}\left|\frac{d \hat{f}_{\xi_{1 / 2}}}{d \mu_{\ell}}(t)\right|^{2} d \tilde{\sigma}_{\ell}\left(t, \xi_{1 / 2}\right)<\infty .
$$

Moreover, the operator $V_{\ell}: \mathcal{H}_{\ell \frac{a}{2}}(T(\Omega)) \rightarrow L^{2}\left(\partial_{\ell} \Omega \times Z_{1 / 2}^{(1 / 2)}, \tilde{\sigma}_{\ell}\right)$ defined via

$$
(V f)\left(t, \xi_{1 / 2}\right)=\left(\frac{\Gamma_{\Omega^{(\ell)}}\left(\ell \frac{a}{2}\right)}{\chi_{\ell}}\right)^{\frac{1}{2}} \frac{d \hat{f}_{\xi_{1 / 2}}}{d \mu_{\ell}}(t)
$$


is a surjective isometry. Thus, for all $f, g \in \mathcal{H}_{\ell \frac{a}{2}}(T(\Omega))$,

$$
\langle f, g\rangle_{\ell \frac{a}{2}}=\frac{\Gamma_{\Omega^{(\ell)}}\left(\ell \frac{a}{2}\right)}{\chi \ell} \int_{N_{\Omega} A\left(u_{\ell}\right)} \int_{Z_{1 / 2}^{(1 / 2)}} \frac{d \hat{f}_{\xi_{1 / 2}}}{d \mu_{\ell}}(t) \frac{\overline{d \hat{g}_{\xi_{1 / 2}}}}{d \mu_{\ell}}(t) d \xi_{1 / 2} N_{\ell}\left(t_{1}\right)^{\ell \frac{a}{2}+b-\frac{d_{1}}{r}} d t_{1} d t_{1 / 2} .
$$

The proof relies on Lemma 2.3.12 and standard techniques (as in the proof of earlier Theorems in this section); it is therefore omitted.

Remark 2.3.14 (1) $\partial_{\ell}(\widehat{(T(\Omega)})$ should not be confused with the boundary orbit $\partial_{\ell}(T(\Omega))$ of $T(\Omega)$ :

$$
\partial_{\ell}(T(\Omega))=\left\{z \in \overline{T(\Omega)} ; \tau(z) \in \partial_{\ell} \Omega\right\}
$$

(2) There is a representation $\varphi \mapsto \tilde{\varphi}$ of $\operatorname{GL}\left(\Omega_{\ell}\right)$ on $Z_{1 / 2}(e)$, defined on the generators of $\operatorname{GL}(\Omega)$ via

$$
\widetilde{P(x)}=R(x), \quad x \in \Omega, \quad \text { and } \quad \tilde{\ell}=\ell, \quad \ell \in L .
$$

One has

$$
\varphi(F(\xi, \eta))=F(\tilde{\varphi}(\xi), \tilde{\varphi}(\eta)), \quad \varphi \in \mathrm{GL}(\Omega), \xi, \eta \in Z_{1 / 2}(e) .
$$

$\mathrm{GL}(\Omega)$ acts also on $\partial_{\ell}(\widehat{T(\Omega)})$ via

$$
\varphi .(t, \xi)=(\varphi(t), \tilde{\varphi}(\xi)), \quad \varphi \in \mathrm{GL}(\Omega), t \in \partial_{\ell}(\Omega), \quad \xi \in Z_{1 / 2}(e) \cap Z_{1 / 2}(s(t)) .
$$

In particular, $\tilde{\varphi}(\xi) \in Z_{1 / 2}(e) \cap Z_{1 / 2}(s(\varphi(t)))$. The proof of Lemma 2.3.8 yields the transformation formula

$$
\sigma_{\ell} \circ \varphi=(\operatorname{Det} \varphi)^{\left(b+\ell \frac{a}{2}\right) / \frac{d_{1}}{r}} \sigma_{\ell} \quad \forall \varphi \in \operatorname{GL}(\Omega)
$$

as well as the Laplace transform formula

$$
\int_{\partial_{\ell}(\widehat{T(\Omega)})} e^{-\langle v+F(\xi) \mid t\rangle} d \sigma_{\ell}(t, \xi)=(2 \pi)^{b \ell+(r-\ell) \ell \frac{a}{2}} \Gamma_{\Omega^{(\ell)}}\left(\ell \frac{a}{2}\right) N(v)^{-\ell \frac{a}{2}}
$$

for all $v \in \Omega$. These properties are analogous to the corresponding properties of $\mu_{\ell}$ (see Theorem 2.2.2).

\subsection{Realization of $\mathcal{H}_{\alpha_{\ell}}(T(\Omega))$ and $\mathcal{H}_{\alpha_{\ell}}(D)$ by boundary integration}

In this section our main concern will be the Wallach points

$$
\alpha_{\ell}=\ell \frac{a}{2}+\frac{d}{r}, \quad 0 \leq \ell \leq r-1 .
$$

Let $D$ be a Cartan domain and let $T(\Omega)$ be the associated symmetric Siegel domain (as in the previous section). We assume that $T(\Omega)$ is of type II; the analysis in the type I case is easier and will follow from the general case.

For $0 \leq \ell \leq r-1$ consider the set

$$
\partial_{\ell}(T(\Omega))=\left\{z \in \overline{T(\Omega)} ; \tau(z) \in \partial_{\ell} \Omega\right\} .
$$


Thus $\partial_{\ell}(T(\Omega))$ consists of all points

$$
z=x+i y+i F(\xi)+\xi, \quad x \in X_{1}(e), \xi \in Z_{\frac{1}{2}}, y \in \partial_{\ell} \Omega .
$$

Hence $\partial_{\ell}(T(\Omega))$ is the direct sum of the Shilov boundary $H$ and $i \partial_{\ell} \Omega$ :

$$
\partial_{\ell}(T(\Omega))=H+i \partial_{\ell} \Omega
$$

We endow $\partial_{\ell}(T(\Omega))$ with the measure

$$
d M_{\ell}^{T(\Omega)}(z)=\varepsilon_{\ell}^{-1} d x d \xi d \mu_{\ell}(y),
$$

where $z=x+i y+i F(\xi)+\xi$ as in (2.4.3) and

$$
\varepsilon_{\ell}=2^{d_{1}-\ell^{2} \frac{a}{2}} \pi^{d+\ell(\ell-r) \frac{a}{2}} \frac{\Gamma_{\Omega^{(\ell)}}\left(\ell \frac{a}{2}\right)}{\Gamma_{\Omega^{(\ell)}}\left(\alpha_{\ell}\right)} .
$$

The reason for including the constant $\varepsilon_{\ell}^{-1}$ in the measure will be clarified by the next lemma. Thus $M_{\ell}^{T(\Omega)}$ is a constant multiple of the product measure $M_{\ell}^{T(\Omega)}=m_{H} \times \mu_{\ell}$, where

$$
d m_{H}(x+i F(\xi)+\xi)=d x d \xi
$$

is the Haar measure of $H$.

Lemma 2.4.1 Fix $w=u+i v+\eta \in T(\Omega)$, with $u \in X_{1}(e), v \in \Omega$ and $\eta \in Z_{\frac{1}{2}}(e)$. Then

$$
\int_{\partial_{\ell}(T(\Omega))}\left|K_{w}^{\left(\alpha_{\ell}\right)}\right|^{2} d M_{\ell}^{T(\Omega)}=K^{\left(\alpha_{\ell}\right)}(w, w) .
$$

Proof: Let $z \in \partial_{\ell}(T(\Omega))$ have the decomposition (2.4.3). Then

$$
\left|K_{w}^{\left(\alpha_{\ell}\right)}(z)\right|^{2}=\left|N\left(x-u+2 \Im F(\xi, \eta)+i\left(y+\frac{1}{2} \tau(w)+F(\xi-\eta)\right)\right)\right|^{-2 \alpha_{\ell}} .
$$

Hence, as in [AU97, Theorem 6.3]

$$
\begin{aligned}
\int_{X}\left|K_{w}^{\left(\alpha_{\ell}\right)}(z)\right|^{2} d x & =\int_{X}\left|N\left(x+i\left(y+\frac{1}{2} \tau(w)+F(\xi-\eta)\right)\right)\right|^{-2 \alpha_{\ell}} d x \\
& =c N\left(y+\frac{1}{2} \tau(w)+F(\xi-\eta)\right)^{-2 \alpha_{\ell}+\frac{d_{1}}{r}}
\end{aligned}
$$

where $d_{1}=\operatorname{dim}_{\mathbb{R}} X_{1}(e)=r(r-1) \frac{a}{2}+r$ and

$$
c=4^{d_{1}-r \alpha_{\ell}} \pi^{d_{1}} \frac{\Gamma_{\Omega}\left(2 \alpha_{\ell}-\frac{d_{1}}{r}\right)}{\Gamma_{\Omega}\left(\alpha_{\ell}\right)^{2}} .
$$

Next, using the formula

$$
N(s)^{-2 \alpha_{\ell}+\frac{d_{1}}{r}}=\frac{1}{\Gamma_{\Omega}\left(2 \alpha_{\ell}-\frac{d_{1}}{r}\right)} \int_{\Omega} e^{-\langle s \mid t\rangle} N(t)^{2 \alpha_{\ell}-2 \frac{d_{1}}{r}} d t
$$


with $s=y+\frac{1}{2} \tau(w)+F(\xi-\eta)$, we obtain

$$
\begin{aligned}
\int_{Z_{\frac{1}{2}}(e)} \int_{X}\left|K_{w}^{\left(\alpha_{\ell}\right)}(z)\right|^{2} d x d \xi & =\frac{c}{\Gamma_{\Omega}\left(2 \alpha_{\ell}-\frac{d_{1}}{r}\right)} \int_{\Omega} e^{-\left\langle y+\frac{1}{2} \tau(w) \mid t\right\rangle} N(t)^{2 \alpha_{\ell}-2 \frac{d_{1}}{r}} d t \int_{Z_{\frac{1}{2}}(e)} e^{-\langle F(\xi-\eta) \mid t\rangle} d \xi \\
& =\frac{(2 \pi)^{r b} c}{\Gamma_{\Omega}\left(2 \alpha_{\ell}-\frac{d_{1}}{r}\right)} \int_{\Omega} e^{-\left\langle y+\frac{1}{2} \tau(w) \mid t\right\rangle} N(t)^{2 \alpha_{\ell}-2 \frac{d_{1}}{r}-b} d t .
\end{aligned}
$$

Thus,

$$
\begin{aligned}
& \int_{\partial_{\ell}(T(\Omega))}\left|K_{w}^{\left(\alpha_{\ell}\right)}(z)\right|^{2} d M_{\ell}^{T(\Omega)}(z)= \\
& =\frac{\varepsilon_{\ell}^{-1}(2 \pi)^{r b} c}{\Gamma_{\Omega}\left(2 \alpha_{\ell}-\frac{d_{1}}{r}\right)} \int_{\Omega} e^{-\left\langle\frac{1}{2} \tau(w) \mid t\right\rangle} N(t)^{2 \alpha_{\ell}-2 \frac{d_{1}}{r}-b} d t \int_{\partial_{\ell} \Omega} e^{-\langle y \mid t\rangle} d \mu_{\ell}(y) \\
& =\frac{\varepsilon_{\ell}^{-1}(2 \pi)^{r b} c \gamma_{\ell}}{\Gamma_{\Omega}\left(2 \alpha_{\ell}-\frac{d_{1}}{r}\right)} \int_{\Omega} e^{-\left\langle\frac{1}{2} \tau(w) \mid t\right\rangle} N(t)^{\alpha_{\ell}-\frac{d_{1}}{r}} d t \\
& =\frac{\varepsilon_{\ell}^{-1}(2 \pi)^{r b} c \gamma_{\ell} \Gamma_{\Omega}\left(\alpha_{\ell}\right)}{\Gamma_{\Omega}\left(2 \alpha_{\ell}-\frac{d_{1}}{r}\right)} N\left(\frac{1}{2} \tau(w)\right)^{-\alpha_{\ell}}=K^{\left(\alpha_{\ell}\right)}(w, w) .
\end{aligned}
$$

For $0 \leq \ell \leq r-1$ we consider the Hardy-type space

$$
H^{2}\left(\partial_{\ell}(T(\Omega))\right)=H^{2}\left(\partial_{\ell}(T(\Omega)), M_{\ell}^{T(\Omega)}\right)
$$

consisting of all holomorphic functions $f$ on $(T(\Omega))$ for which

$$
\|f\|_{H^{2}\left(\partial_{\ell}(T(\Omega))\right)}^{2}:=\sup _{t \in \Omega} \int_{\partial_{\ell}(T(\Omega))}|f(z+i t)|^{2} d M_{\ell}^{T(\Omega)}(z)
$$

is finite. Standard arguments show that for $f \in H^{2}\left(\partial_{\ell}(T(\Omega))\right)$ the boundary values

$$
f(z):=\lim _{\Omega \ni t \rightarrow 0} f(z+i t), \quad z \in \partial_{\ell}(T(\Omega))
$$

exist almost everywhere, and

$$
\begin{aligned}
\|f\|_{H^{2}\left(\partial_{\ell}(T(\Omega))\right)}^{2} & =\lim _{\Omega \ni t \rightarrow 0} \int_{\partial_{\ell}(T(\Omega))}|f(z+i t)|^{2} d M_{\ell}^{T(\Omega)}(z) \\
& =\int_{\partial_{\ell}(T(\Omega))}|f(z)|^{2} d M_{\ell}^{T(\Omega)}(z) .
\end{aligned}
$$

See the proof of Theorem 6.3 in [AU97].

Theorem 2.4.2 For $0 \leq \ell \leq r-1$ we have $\mathcal{H}_{\alpha_{\ell}}=H^{2}\left(\partial_{\ell}(T(\Omega))\right)$, and moreover

$$
\|f\|_{\alpha_{\ell}}=\|f\|_{H^{2}\left(\partial_{\ell}(T(\Omega))\right)}^{2}, \quad \forall f \in \mathcal{H}_{\alpha_{\ell}}(T(\Omega)) .
$$


Thus, for all $f, g \in \mathcal{H}_{\alpha_{\ell}}$

$$
<f, g>_{\alpha_{\ell}}=\lim _{\Omega \ni t \rightarrow 0} \int_{\partial_{\ell}(T(\Omega))} f(z+i t) \overline{g(z+i t)} d M_{\ell}^{T(\Omega)}(z) .
$$

Theorem 2.4.2 is the generalization of Theorem 6.3 of [AU97] to symmetric Siegel domains of type II. The proof uses Lemma 2.4.1 (which yields (2.4.12) and (2.4.13) for functions in $\left.\mathcal{H}_{\alpha_{\ell}}(T(\Omega))^{(0)}=\operatorname{span}\left\{K_{w}^{\left(\alpha_{\ell}\right)} ; w \in T(\Omega)\right\}\right)$ as well as the standard arguments used in the proofs of the theorems in Section 6 and in the proof of Theorem 6.3 in [AU97].

Notice that, in particular, the reproducing kernel of $H^{2}\left(\partial_{\ell}(T(\Omega))\right)$ is

$$
K^{\left(\alpha_{\ell}\right)}(z, w)=N(\tau(z, w))^{-\alpha_{\ell}}, \quad z \in \partial_{\ell}(T(\Omega)), w \in T(\Omega) .
$$

Consider the inverse Cayley transform $c^{-1}: T(\Omega) \rightarrow D$,

$$
c^{-1}(w):=\frac{w_{1}-i e}{w_{1}+i e}+\sqrt{2} i R\left(\left(w_{1}+i e\right)^{-1}\right) w_{\frac{1}{2}}
$$

(where $\left.w=w_{1}+w_{\frac{1}{2}}, w_{1} \in Z_{1}(e), w_{\frac{1}{2}} \in Z_{\frac{1}{2}}(e)\right) . c^{-1}$ extends to $\partial(T(\Omega))=\{w \in \overline{T(\Omega)} ; \tau(w) \in$ $\partial \Omega\}$, and it maps holomorphic boundary components of $T(\Omega)$ to holomorphic boundary components of $D$, and preserves the rank of the boundary components. But not every holomorphic boundary component $B(v)=v+D_{0}(v)$ of $D$ is obtained in this way, since $c(B(v))=\infty$ if $e-v$ is not invertible in $Z_{1}(e)$. Thus

$$
c^{-1}\left(\partial_{\ell}(T(\Omega))\right)=\bigcup_{\substack{v \in S_{r-\ell} \\ e-v \text { invertible }}} B(v) \varsubsetneqq \partial_{r-\ell}(D) .
$$

On the set $c^{-1}\left(\partial_{\ell}(T(\Omega))\right)$ consider the measure

$$
d M_{\ell}^{D}(z):=|J c(z)|^{-\frac{2 \alpha_{\ell}}{p}} d M_{\ell}^{T(\Omega)}(c(z)) .
$$

Then $M_{\ell}^{D}$ is absolutely continuous with respect to the volume measure on $c^{-1}\left(\partial_{\ell}(T(\Omega))\right)$. Since $\partial_{r-\ell}(D) \backslash c^{-1}\left(\partial_{\ell}(T(\Omega))\right)$ is a lower dimensional subset of $\partial_{r-\ell}(D)$, its volume measure is zero. This consideration enables us to consider $M_{\ell}^{D}$ as an absolutely continuous measure on all of $\partial_{r-\ell}(D)$ in a unique way. In Section 3.5 this boundary measure will be constructed directly and analyzed in more detail.

The Hardy space

$$
H^{2}\left(\partial_{r-\ell}(D)\right)=H^{2}\left(\partial_{r-\ell}(D), M_{\ell}^{D}\right)
$$

is the space of all holomorphic functions in $D$ for which

$$
\|f\|_{H^{2}\left(\partial_{r-\ell}(D)\right)}^{2}:=\sup _{0<t<1} \int_{\partial_{r-\ell}(D)}|f(t z)|^{2} d M_{\ell}^{D}(z)
$$

is finite. By standard arguments, for each $f \in H^{2}\left(\partial_{r-\ell}(D)\right)$ the radial limit (here $f_{t}(z):=$ $f(t z))$

$$
f_{1}(z)=\lim _{t \rightarrow 1-} f_{t}(z), \quad z \in \partial_{r-\ell}(D)
$$


exists in $L^{2}\left(\partial_{r-\ell}(D)\right)$ and almost everywhere on $\partial_{r-\ell}(D)$. Moreover

$$
\|f\|_{H^{2}\left(\partial_{r-\ell}(D)\right)}=\lim _{t \rightarrow 1-}\left\|f_{t}\right\|_{L^{2}\left(\partial_{r-\ell}(D)\right)}\left\|f_{1}\right\|_{L^{2}\left(\partial_{r-\ell}(D)\right)} .
$$

Recall that the operator

$$
f \mapsto(f \circ c)(J c)^{\alpha_{\ell} / p}
$$

maps $\mathcal{H}_{\alpha_{\ell}}(T(\Omega))$ isometrically onto $\mathcal{H}_{\alpha_{\ell}}(D)$. Therefore Theorem 2.4.2 enables us to obtain the following result.

Theorem 2.4.3 For $0 \leq \ell \leq r-1$ we have $\mathcal{H}_{\alpha_{\ell}}(D)=H^{2}\left(\partial_{r-\ell}(D), M_{\ell}^{D}\right)$ and

$$
\|f\|_{\alpha_{\ell}}=\|f\|_{H^{2}\left(\partial_{r-\ell}(D), M_{\ell}^{D}\right)} \quad \forall f \in \mathcal{H}_{\alpha_{\ell}}(D) .
$$

Thus, for all $f, g \in \mathcal{H}_{\alpha_{\ell}}(D)$

$$
<f, g>_{\alpha_{\ell}}=\lim _{t \rightarrow 1-} \int_{\partial_{r-\ell}(D)} f(t z) \overline{g(t z)} d M_{\ell}^{D}(z) .
$$

Theorems (2.1.3) and (2.4.3) combine to yield the following result.

Theorem 2.4.4 Let $0 \leq \ell \leq r-1$ and, as before, let $\alpha_{\ell}=\ell \frac{a}{2}+\frac{d}{r}$. Then there exists an operator $T$ on $C^{\infty}\left(D \cup \partial_{r-\ell}(D)\right)$ which is $\mathrm{GL}\left(\Omega^{(\ell)}\right)$-invariant, so that

(i) For every $f \in \mathcal{H}_{\ell_{\frac{a}{2}}}(D)$ with Peter-Weyl expansion $f=\sum_{\mathbf{m}^{(\ell)}} f_{\mathbf{m}^{(\ell)}}$, one has

$$
T f=\sum_{\mathbf{m}^{(\ell)}} \frac{\left(\alpha_{\ell}\right)_{\mathbf{m}^{(\ell)}}}{\left(\ell \frac{a}{2}\right)_{\mathbf{m}^{(\ell)}}} f_{\mathbf{m}^{(\ell)}} .
$$

(ii) For all $f, g \in \mathcal{H}_{\ell \frac{a}{2}}(D)$,

$$
<f, g>_{\ell \frac{a}{2}}=<T f, g>_{H^{2}\left(\partial_{r-\ell}(D)\right)}=\lim _{t \rightarrow 1-} \int_{\partial_{r-\ell}(D)} T(f \bar{g}) d M_{\ell}^{D} .
$$

The volume measure $m$ on $\partial_{r-\ell}(D)$ is given by

$$
\int_{\partial_{r-\ell}(D)} f d m=\int_{S_{r-\ell}} d \nu_{r-\ell}(v) \int_{D_{0}(v)} f_{v}(z) d m_{v}(z)
$$

where $m_{v}$ is the Lebesgue measure on $D_{0}(v)$. Let us consider the Radon-Nikodym derivative

$$
\omega(z)=\frac{d M_{\ell}^{D}}{d m}(z), \quad z \in \partial_{r-\ell}(D) .
$$

Then formula (2.4.25) can be written in the form

$$
\begin{aligned}
<f, g>_{\ell \frac{a}{2}} & =\int_{S_{r-\ell}} d \nu_{r-\ell}(v) \int_{D_{0}(v)} T_{v}\left(f_{v}\right)(z) \overline{g_{v}(z)} \omega(v+z) d m_{v}(z) \\
& =\int_{S_{r-\ell}} d \nu_{r-\ell}(v) \int_{D_{0}(v)} T_{v}\left(f_{v} \overline{g_{v}}\right)(z) \omega(v+z) d m_{v}(z) .
\end{aligned}
$$




\section{Semi-invariant Measures on Boundary Orbits}

The main results of Section 2 are mostly expressed as boundary integration formulas for invariant inner products, using canonical "semi-invariant" measures supported on the various group orbits in the boundary of the underlying domain. In the following Sections we analyze these boundary measures and their main features (existence, polar decomposition etc.) in more detail, using only the basic properties of Jordan algebras and triples. In the unbounded setting of Siegel domains and tube domains, the boundary measures were constructed by Lassalle [La87], but here we emphasize the corresponding polar decomposition which makes the symmetry properties more transparent. In the bounded case, the relevant boundary measures are also constructed from first principles avoiding extensive use of the Cayley transform. As an application we obtain an independent proof of the main result of Section 2.4 in the bounded setting (cf. Theorem 3.4.6).

\subsection{General formulas for homogeneous spaces}

In the following we consider locally compact groups $G$, with left Haar measure denoted by $\mu_{G}$ and modulus function denoted by $\Delta_{G}$. For $f \in \mathcal{C}_{c}(G)$ let

$$
\left(\ell_{g} f\right)(x):=f\left(g^{-1} x\right)
$$

and

$$
\left(\delta_{g} f\right)(x):=f(x g)
$$

denote left and right translation by $g \in G$, resp. Throughout, we will use the concept of "quotient measures" on homogeneous spaces [Bou63, p. 44, Définition 1].

Proposition 3.1.1 Let $G$ be a locally compact group, with closed subgroups $P \subset Q \subset G \supset K$ such that $G=K Q$ and

$$
\Delta_{K}(k)=\Delta_{K \cap Q}(k) \quad \forall k \in K \cap Q
$$

and

$$
\Delta_{Q}(p)=\Delta_{P}(p) \quad \forall p \in P .
$$

i) Let $\chi: G \rightarrow \mathbb{R}_{>}$be a continuous function satisfying

$$
\delta_{p} \chi=\frac{\Delta_{P}(p)}{\Delta_{G}(p)} \chi \quad \forall p \in P
$$

and

$$
\ell_{k} \chi=\chi \quad \forall k \in K
$$

Then the (well-defined) quotient measures

$$
\begin{aligned}
\mu_{K / K \cap Q} & :=\frac{\mu_{K}}{\mu_{K \cap Q}} \text { on } \quad K / K \cap Q, \\
\mu_{Q / P} & :=\frac{\mu_{Q}}{\mu_{P}} \text { on } Q / P, \\
\mu_{G / P} & :=\frac{\chi \mu_{G}}{\mu_{P}} \text { on } G / P
\end{aligned}
$$


yield a decomposition

$$
\int_{G / P} d \mu_{G / P} f=\int_{K / K \cap Q} d \mu_{K / K \cap Q}(k K \cap Q) \cdot \int_{Q / P} d \mu_{Q / P}(q P) f(k q P) \frac{\chi(q) \Delta_{G}(q)}{\Delta_{Q}(q)}
$$

for all $f \in \mathcal{C}_{c}(G / P)$.

ii) Let $\chi$ be a positive character of $G$ satisfying

$$
\chi(p)=\frac{\Delta_{P}(p)}{\Delta_{G}(p)} \quad \forall p \in P
$$

and

$$
\chi(k)=1 \quad \forall k \in K .
$$

Then (3.1.5) and (3.1.6) hold, and $\mu_{G / P}$ is relatively invariant with multiplier $\chi$.

Proof: By (3.1.3) and (3.1.6), $K / K \cap Q$ carries a $K$-invariant measure

$$
\mu_{K / K \cap Q}=\frac{\mu_{K}}{\mu_{K \cap Q}} .
$$

Since $G / Q \approx K / K \cap Q$, there exists a function $\rho: G \rightarrow \mathbb{R}_{>}$satisfying

$$
\delta_{q} \rho=\frac{\Delta_{Q}(q)}{\Delta_{G}(q)} \rho \quad \forall q \in Q
$$

giving rise to the quotient measure

$$
\frac{\rho \mu_{G}}{\mu_{Q}}=\mu_{K / K \cap Q}
$$

[Bou63, p. 56, Lemme 5]. By $K$-invariance, we have

$$
\ell_{k} \rho=\rho \quad \forall k \in K .
$$

It follows that

$$
\rho(g)=\frac{\Delta_{Q}(q)}{\Delta_{G}(q)}
$$

whenever $g \in G, q \in Q$ satisfy $g^{-1} K=q^{-1} K$. By (3.1.4) and [Bou63, p. 59, Corollaire], $Q / P$ carries a $Q$-invariant measure

$$
\mu_{Q / P}=\frac{\mu_{Q}}{\mu_{P}} .
$$

In view of (3.1.4) and (3.1.13), the quotient measure $\rho \mu_{G} / \mu_{P}$ on $G / P$ exists and, by [Bou63, p.63, Proposition 12], there is a decomposition

$$
\begin{gathered}
\int_{G / P} d\left(\frac{\rho \mu_{G}}{\mu_{P}}\right) f=\int_{G / Q} d \mu_{K / K \cap Q}(g Q) \int_{Q / P} d \mu_{Q / P} \ell_{g^{-1}} f= \\
\int_{G / Q} d \mu_{K / K \cap Q}(g Q) \int_{Q / P} d \mu_{Q / P}(q P) f(g q P)= \\
\int_{K / K \cap Q} d \mu_{K / K \cap Q}(k K \cap Q) \int_{Q / P} d \mu_{Q / P}(q P) f(k q P)
\end{gathered}
$$


for $f \in \mathcal{C}_{c}(G / P)$. By (3.1.5), (3.1.13) and (3.1.4) we have

$$
\delta_{p} \frac{\chi}{\rho}=\frac{\delta_{p} \chi}{\delta_{p} \rho}=\frac{\Delta_{P}(p) \chi \cdot \Delta_{G}(p)}{\Delta_{G}(p) \Delta_{Q}(p) \rho}=\frac{\chi}{\rho}
$$

for all $p \in P \subset Q$. Therefore, $\chi / \rho$ is a function on $G / P$ and we have

$$
\mu_{G / P}=\frac{\chi \mu_{G}}{\mu_{P}}=\frac{\chi}{\rho} \frac{\rho \mu_{G}}{\mu_{P}}
$$

as measures on $G / P$. Applying (3.1.18) to $f \chi / \rho \in \mathcal{C}_{c}(G / P)$ one obtains

$$
\begin{aligned}
& \int_{G / P} d \mu_{G / P} f=\int_{G / P} d\left(\frac{\rho \mu_{G}}{\mu_{P}}\right) \frac{\chi}{\rho} f= \\
& \int_{K / K \cap Q} d \mu_{K / K \cap Q}(k K \cap Q) \int_{Q / P} d \mu_{Q / P}(q P) \varphi(k q P) \frac{\chi(k q)}{\rho(k q)} .
\end{aligned}
$$

Since $\chi(k q)=\chi(q)$ by $(3.1 .6)$ and

$$
\rho(k q)=\frac{\Delta_{Q}(q)}{\Delta_{G}(q)} \rho(k)=\frac{\Delta_{Q}(q)}{\Delta_{G}(q)} \rho(e)
$$

by (3.1.13) and (3.1.15), the assertion follows if we normalize $\rho(e)=1$.

Proposition 3.1.2 Let $g \in G, q \in Q$ satisfy $g^{-1} K=q^{-1} K$. Then the Radon-Nikodym density is given by

$$
\frac{d \mu_{K / K \cap Q}(g Q)}{d \mu_{K / K \cap Q}(Q)}=\frac{\Delta_{Q}(q)}{\Delta_{G}(q)}
$$

Proof: By [Bou63, p. 54, Lemme 4] we have

$$
\begin{aligned}
\int_{K / K \cap Q} d\left(g_{*}^{-1} \mu_{K / K \cap Q}\right) f & =\int_{K / K \cap Q} d \mu_{K / K \cap Q} \ell_{g} f=\int_{K / K \cap Q} d\left(\frac{\rho \mu_{G}}{\mu_{Q}}\right) \ell_{g} f \\
& =\int_{K / K \cap Q} d\left(\frac{\rho \mu_{G}}{\mu_{Q}}\right) \frac{\ell_{g^{-1}} \rho}{\rho} f=\int_{K / K \cap Q} d \mu_{K / K \cap Q} \frac{\ell_{g^{-1}} \rho}{\rho} f .
\end{aligned}
$$

Therefore

$$
\frac{d \mu_{K / K \cap Q}(g x Q)}{d \mu_{K / K \cap Q}(x Q)}=\frac{\ell_{g^{-1}} \rho}{\rho}(x Q)=\frac{\rho(g x)}{\rho(x)}
$$

for all $x \in G$. Since $g=k q$ for some $k \in K$, we obtain

$$
\frac{d \mu_{K / K \cap Q}(g Q)}{d \mu_{K / K \cap Q}(Q)}=\rho(g)=\rho(k q)=\left(\delta_{q} \rho\right)(k)=\frac{\Delta_{Q}(q)}{\Delta_{G}(q)} \rho(k)=\frac{\Delta_{Q}(q)}{\Delta_{G}(q)} .
$$


Proposition 3.1.3 Let $G$ be a (not necessarily connected) Lie group having a compact subgroup $K$ such that $G / K$ is connected. Let

$$
\mathfrak{g}=\sum_{\alpha}^{\oplus} \mathfrak{g}_{\alpha}=\mathfrak{g}_{0} \oplus \sum_{\alpha \neq 0}^{\oplus} \mathfrak{g}_{\alpha}
$$

be a direct sum decomposition of $\mathfrak{g}$ satisfying

$$
\left[\mathfrak{g}_{\alpha}, \mathfrak{g}_{\beta}\right] \subset \mathfrak{g}_{\alpha+\beta} \quad \forall \alpha, \beta
$$

and

$$
\mathfrak{g}_{0}=\mathfrak{a} \oplus \mathfrak{m}
$$

with

$$
\mathfrak{m} \subset \mathfrak{k} .
$$

Let $\chi: G \rightarrow \mathbb{R}_{>}$be a character satisfying

$$
\left.d \chi\right|_{\mathfrak{g}_{\alpha}}=0 \quad \forall \alpha \neq 0
$$

and

$$
\operatorname{tr} a d_{\mathfrak{g}}(\gamma)=c d \chi(\gamma) \quad \forall \gamma \in \mathfrak{a}
$$

where $c$ is a fixed constant. Then

$$
\Delta_{G}(g)=\chi(g)^{-c} \quad \forall g \in G .
$$

Proof: By (3.1.28) we have

$$
\operatorname{tr} a d_{\mathfrak{g}}(\gamma)=0
$$

for all $\gamma \in \mathfrak{g}_{\alpha}$ such that $\alpha \neq 0$. Consider the character

$$
\eta(g):=\chi(g)^{c} \Delta_{G}(g) .
$$

Since $\eta(K) \subset \mathbb{R}_{>}$is a compact subgroup it follows that $\left.\eta\right|_{K}=1$. With (3.1.30) this implies

$$
\left.d \eta\right|_{\mathfrak{m}}=0 .
$$

Applying [Dieu74, (19.16.4.3)] we have

$$
\Delta_{G}(\exp \gamma)^{-1}=\operatorname{Det} A d_{\mathfrak{g}}(\exp \gamma)=\operatorname{Det} \exp a d_{\mathfrak{g}}(\gamma)=\exp \operatorname{tr} a d_{\mathfrak{g}}(\gamma)
$$

for all $\gamma \in \mathfrak{g}$. Therefore

$$
d \eta(\gamma)=c \cdot d \chi(\gamma)-\operatorname{tr} a d_{\mathfrak{g}}(\gamma),
$$

and hence $\left.d \eta\right|_{\mathfrak{g}_{\alpha}}=0$ by (3.1.31) and (3.1.34), whereas $\left.d \eta\right|_{\mathfrak{a}}=0$ by (3.1.32). In view of (3.1.27) it follows that $d \eta=0$. Now let $g \in G$. Since $G / K$ is connected, there exists $k \in K$ such that $g k^{-1} \in G^{0}$ (identity component). Writing

$$
g k^{-1}=\exp \left(\gamma_{1}\right) \cdots \exp \left(\gamma_{n}\right)
$$

for suitable $\gamma_{1}, \ldots, \gamma_{n} \in \mathfrak{g}$, we obtain

$$
\eta(g)=\eta\left(\exp \left(\gamma_{1}\right)\right) \cdots \eta\left(\exp \left(\gamma_{n}\right)\right) \eta(k)=\exp \left(d \eta\left(\gamma_{1}\right)\right) \cdots \exp \left(d \eta\left(\gamma_{n}\right)\right)=1 .
$$

In the following these elementary results will be applied to various homogeneous spaces related to Jordan algebras and symmetric domains. 


\subsection{Boundary orbits of symmetric cones}

Let $X$ be an irreducible euclidean Jordan algebra of rank $r$, with open positive cone $\Omega$ and unit element $e$. Then

$$
G:=G L(\Omega)
$$

is a reductive Lie group, with Lie algebra denoted by $\mathfrak{g}=\mathfrak{g l}(\Omega)$. A maximal compact subgroup is

$$
K=\operatorname{Aut}(X)=\{g \in G: g(e)=e\},
$$

with Lie algebra $\mathfrak{k}=$ aut $(X)$ consisting of all Jordan algebra derivations. Using the Jordan triple product, put

$$
\left(u \square v^{*}\right) x:=\left\{u v^{*} x\right\}
$$

for all $u, v, x \in X$. Let $\Delta$ be the norm function ("determinant") of $X$, and let $(x \mid y)$ be the $K$-invariant inner product, normalized by $(e \mid e)=r$.

Lemma 3.2.1 $\chi(g):=\Delta(g e)$ defines a character of $G$, with differential

$$
d \chi(\gamma)=(e \mid \gamma e) \quad \forall \gamma \in \mathfrak{g} .
$$

Proof: Since $\Delta(g x)=\Delta(g e) \Delta(x)$ for all $g \in G$ and $x \in X$, we have $\Delta\left(g_{1} g_{2} e\right)=\Delta\left(g_{1} e\right) \Delta\left(g_{2} e\right)$ for all $g_{1}, g_{2} \in G$. Thus $\chi$ is a character. Since $\Delta^{\prime}(e) x=(e \mid x)$ for all $x \in X$, its differential is

$$
d \chi(\gamma)=\left.\frac{d}{d t}\right|_{t=0} \chi(\exp t \gamma)=\left.\frac{d}{d t}\right|_{t=0} \Delta(\exp (t \gamma) e)=\Delta^{\prime}(e) \gamma e=(e \mid \gamma e) .
$$

Fix a frame $\left\{e_{1}, \ldots, e_{r}\right\}$ of minimal orthogonal idempotents in $X$, and consider the associated Peirce decomposition

$$
X=\sum_{1 \leq i \leq j \leq r}^{\oplus} X_{i j}
$$

With respect to the Cartan subspace

$$
\mathfrak{a}:=\left\langle e_{k} \square \stackrel{*}{e}_{k}: 1 \leq k \leq r\right\rangle \quad \text { (real span) }
$$

$\mathfrak{g}$ has a real root decomposition

$$
\mathfrak{g}=\mathfrak{a} \oplus \mathfrak{m} \oplus \sum_{1 \leq i<j \leq r}\left(\mathfrak{g}_{j-i}^{+} \oplus \mathfrak{g}_{j-i}^{-}\right)
$$

where

$$
\begin{aligned}
\mathfrak{m} & :=\left\{\delta \in \operatorname{aut}(X): \delta e_{k}=0 \quad \forall 1 \leq k \leq r\right\} \\
\mathfrak{g}_{j-i}^{+} & :=\left\{a \square \stackrel{*}{e}_{i}=e_{j} \square \stackrel{*}{a}: a \in X_{i j}\right\}, \\
\mathfrak{g}_{j-i}^{-} & :=\left\{a \square \stackrel{*}{e}_{j}=e_{i} \square \stackrel{*}{a}: a \in X_{i j}\right\} .
\end{aligned}
$$

For $m \in\{i, j\}$ we have

$$
\begin{aligned}
& {\left[e_{k} \square \stackrel{*}{e}_{k}, e_{m} \square \stackrel{*}{a}\right]=\left\{e_{k} \stackrel{*}{e}_{k} e_{m}\right\} \square \stackrel{*}{a}-e_{m} \square\left\{a \stackrel{*}{e}_{k} e_{k}\right\}^{*}} \\
& \quad=\delta_{m k} e_{m} \square \stackrel{*}{a}-\frac{\delta_{i k}+\delta_{j k}}{2} e_{m} \square \stackrel{*}{a}=\left(\delta_{m k}-\frac{\delta_{i k}+\delta_{j k}}{2}\right) e_{m} \square \stackrel{*}{a},
\end{aligned}
$$


as follows from the Jordan triple identity [U87]. Therefore $\mathfrak{g}_{j-i}^{\kappa}$ is the root space corresponding to the root $\frac{\kappa}{2}\left(\delta_{j}-\delta_{i}\right)$ defined by

$$
\kappa \frac{\delta_{j}-\delta_{i}}{2}\left(e_{k} \square e_{k}^{*}\right):=\kappa \frac{\delta_{j k}-\delta_{i k}}{2} \quad \forall k
$$

We now turn to the Iwasawa decomposition

$$
G=G L(\Omega)=A u t(X) A N
$$

of the (reductive) Lie group $G L(\Omega)$. The Lie algebra of $N$ is given by

$$
\mathfrak{n}=\sum_{1 \leq i<j \leq r} \mathfrak{g}_{j-i}^{+}
$$

Similarly,

$$
\overline{\mathfrak{n}}=\sum_{1 \leq i<j \leq r} \mathfrak{g}_{j-i}^{-}
$$

$A N$ and $A \bar{N}$ act (simply) transitively on $\Omega$.

Lemma 3.2.2 Let $\gamma=\sum_{k} \gamma^{k} e_{k} \square \stackrel{*}{e}_{k} \in \mathfrak{a}$. Then

$$
\operatorname{tr} a d_{\mathfrak{a} \oplus \mathfrak{n}}(\gamma)=\operatorname{tr} a d_{\mathfrak{n}}(\gamma)=\frac{a}{2} \sum_{k} \gamma^{k}(2 k-r-1) .
$$

Proof: Counting with multiplicity, we obtain

$$
\operatorname{tr} a d_{\mathfrak{n}}(\gamma)=a \sum_{i<j} \frac{\gamma^{j}-\gamma^{i}}{2}=\frac{a}{2} \sum_{k} \gamma^{k}(2 k-r-1)
$$

Proposition 3.2.3 AN has the modulus function

$$
\Delta_{A N}(g)=\Delta_{-2 \rho}(g e),
$$

where

$$
\rho_{k}:=\frac{a}{4}(2 k-r-1) .
$$

Proof: For $1 \leq k \leq r$, the minors $\Delta_{k}$ satisfy

$$
\Delta_{k}(g x)=\Delta_{k}(g e) \Delta_{k}(x)
$$

for all $g \in A N$. Therefore $\Delta_{k}\left(g_{1} g_{2} e\right)=\Delta_{k}\left(g_{1} e\right) \Delta_{k}\left(g_{2} e\right)$ for all $g_{1}, g_{2} \in A N$, showing that

$$
\chi_{k}(g):=\Delta_{k}(g e)
$$

defines a character of $A N$. For $\gamma \in \mathfrak{a} \oplus \mathfrak{n}$, its differential is

$$
\begin{aligned}
& d \chi_{k}(\gamma)=\left.\frac{d}{d t}\right|_{t=0} \chi_{k}(\exp (t \gamma))= \\
& \left.\quad \frac{d}{d t}\right|_{t=0} \Delta_{k}(\exp (t \gamma) e)=\Delta_{k}^{\prime}(e)(\gamma e)=\left(e_{1}+\cdots+e_{k} \mid \gamma e\right)
\end{aligned}
$$


since $\Delta_{k}^{\prime}(e) x=\left(e_{1}+\cdots+e_{k} \mid x\right)$ for all $x \in X$. It follows that

$$
\chi(g):=\Delta_{-2 \rho}(g e)=\chi_{r}(g)^{-\frac{a}{2}(r-1)} \prod_{k<r} \chi_{k}(g)^{a}
$$

defines a character of $A N$, with differential

$$
\begin{aligned}
& d \chi(\gamma)=\sum_{k<r} a d \chi_{k}(\gamma)-\frac{a}{2}(r-1) \cdot d \chi_{r}(\gamma)= \\
& \quad a \sum_{k<r}\left(e_{1}+\cdots+e_{k} \mid \gamma e\right)-\frac{a}{2}(r-1)(e \mid \gamma e)=-\frac{a}{2} \sum_{k=1}^{r}(2 k-r-1)\left(e_{k} \mid \gamma e\right) .
\end{aligned}
$$

If $i<j$ and $a \in X_{i j}$, then $\gamma:=e_{j} \square \stackrel{*}{a} \in \mathfrak{g}_{j-i}^{+}$and $\left(e_{k} \mid \gamma e\right)=\left(e_{k} \mid\left\{e_{j} \stackrel{*}{a} e\right\}\right)=\frac{1}{2}\left(e_{k} \mid a\right)=0$. If $\gamma=\sum_{j} \gamma^{j} e_{j} \square \stackrel{*}{e}_{j} \in \mathfrak{a}$ then $\gamma e=\sum_{j} \gamma^{j} e_{j}$ and Lemma 3.2 .2 implies

$$
d \chi(\gamma)=-\frac{a}{2} \sum_{k=1}^{r}(2 k-r-1) \gamma^{k}=-t r a d_{\mathfrak{a} \oplus \mathfrak{n}}(\gamma) .
$$

Now apply Proposition 3.1.3 to $G:=A N$ and $K:=\{1\}$.

Fix $0 \leq \ell<r$ and put $u_{\ell}=e_{1}+\cdots+e_{\ell}$. Then

$$
\partial_{\ell} \Omega:=\{x \in \partial \Omega: \operatorname{rank}(x)=\ell\}=G \cdot u_{\ell}
$$

is a $G$-orbit, i.e., we may identify

$$
G / P \ni g P \underset{\approx}{\rightleftarrows} g u_{\ell} \in \partial_{\ell} \Omega
$$

where

$$
P:=\left\{p \in G: p u_{\ell}=u_{\ell}\right\}
$$

is a closed subgroup of $G$, with Lie algebra

$$
\wp:=\left\{\gamma \in \mathfrak{g}: \gamma u_{\ell}=0\right\} .
$$

Let $\Pi_{\ell}$ denote the compact manifold of all idempotents of rank $\ell$ in $X$. For $u \in \Pi_{\ell}$, let $\Omega_{1}(u)$ denote the positive cone of the euclidean Jordan algebra $X_{1}(u)$ of rank $\ell$. Then

$$
\partial_{\ell} \Omega=\bigcup_{u \in \Pi_{\ell}} \Omega_{1}(u) \quad \text { (disjoint union). }
$$

Since $G$ permutes the fibres of (3.2.29), there exists a (non-linear) action $g \mapsto \widetilde{g}$ of $G$ on $\Pi_{\ell}$ satisfying

$$
g \Omega_{1}(u)=\Omega_{1}(\widetilde{g}(u))
$$

for all $u \in \Pi_{\ell}$. This action is transitive, and hence there exists a diffeomorphism

$$
G / Q \ni g Q \mapsto \widetilde{g}\left(u_{\ell}\right) \in \Pi_{\ell}
$$


where

$$
Q=\left\{q \in G: \widetilde{q}\left(u_{\ell}\right)=u_{\ell}\right\}=\left\{q \in G: q u_{\ell} \in \Omega_{1}\left(u_{\ell}\right)\right\}
$$

is a closed subgroup of $G$ containing $P$, with Lie algebra

$$
q=\left\{\gamma \in \mathfrak{g}: \gamma u_{\ell} \in X_{1}\left(u_{\ell}\right)\right\} .
$$

If $q \in Q \cap K$ then $q u_{\ell} \in \Pi_{\ell} \cap \Omega_{1}\left(u_{\ell}\right)=\left\{u_{\ell}\right\}$ and hence $q \in P$. Thus $Q \cap K=P \cap K$. Evaluating at $u_{\ell}$, we obtain

$$
\delta u_{\ell}=\sum_{k \leq \ell} \delta e_{k}=0 \quad \text { for all } \quad \delta \in \mathfrak{m}
$$

whereas for $a \in X_{i j}$ we have

$$
\begin{aligned}
& \left\{e_{k} e_{k}^{*} u_{\ell}\right\}=\left\{\begin{array}{ll}
e_{k} & k \leq \ell \\
0 & k>\ell
\end{array},\right. \\
& \left\{e_{j} \stackrel{*}{a} u_{\ell}\right\}=\left\{\begin{array}{ll}
\frac{a}{2} & \ell \geq i<j \\
0 & \ell<i<j
\end{array},\right. \\
& \left\{a \stackrel{*}{e}_{j} u_{\ell}\right\}=\left\{\begin{array}{ll}
\frac{a}{2} & i<j \leq \ell \\
0 & i<j>\ell
\end{array} .\right.
\end{aligned}
$$

It follows that

$$
\begin{gathered}
\wp=\mathfrak{m} \oplus\left\langle e_{k} \square e_{k}^{*}: k>\ell\right\rangle \oplus \sum_{\ell<i<j} \mathfrak{g}_{j-i}^{+} \oplus \sum_{i<j>\ell} \mathfrak{g}_{j-i}^{-}, \\
\mathfrak{q}=\wp \oplus\left\langle e_{k} \square e_{k}^{*}: k \leq \ell\right\rangle \oplus \sum_{1 \leq i<j \leq \ell}\left(\mathfrak{g}_{j-i}^{+} \oplus \mathfrak{g}_{j-i}^{-}\right) .
\end{gathered}
$$

This implies $\mathfrak{q} \supset \mathfrak{a} \oplus \overline{\mathfrak{n}}$ and $Q \supset A \bar{N}$.

Lemma 3.2.4 $Q$ has the modulus function

$$
\Delta_{Q}(q)=\Delta(q e)^{(\ell-r) a / 2} \bar{\Delta}_{r-\ell}(q e)^{r a / 2} .
$$

Proof: $\chi(q):=\Delta(q e)^{\ell-r} \bar{\Delta}_{r-\ell}(q e)^{r}$ defines a character of $A \bar{N} \subset Q$, with differential

$$
\begin{aligned}
& d \chi(\gamma)=(\ell-r) \Delta^{\prime}(e) \gamma e+r \bar{\Delta}_{r-\ell}(e) \gamma e \\
& \quad=(\ell-r)(e \mid \gamma e)+r\left(e_{\ell+1}+\cdots+e_{r} \mid \gamma e\right)
\end{aligned}
$$

for all $\gamma \in \mathfrak{a} \oplus \overline{\mathfrak{n}}$. If $\gamma=a \square e_{j}^{*} \in \overline{\mathfrak{n}}$, then $\gamma e=\frac{a}{2}$ and $d \chi(\gamma)=0$ since $i<j$. If $\gamma=$ $\sum_{k} \gamma^{k} e_{k} \square e_{k}^{*} \in \mathfrak{a}$ then $\gamma e=\sum_{k} \gamma^{k} e_{k}$ and

$$
d \chi(\gamma)=(\ell-r) \sum_{k \leq r} \gamma^{k}+r \sum_{k>\ell} \gamma^{k}=(\ell-r)\left(\gamma^{1}+\cdots+\gamma^{\ell}\right)+\ell \sum_{k>\ell} \gamma^{k} .
$$

On the other hand, (3.2.36) and (3.2.36) imply

$$
\operatorname{tr} a d_{\mathfrak{q}} \gamma=\sum_{\ell<i<j} \frac{a}{2}\left(\gamma^{j}-\gamma^{i}\right)+\sum_{i<j>\ell} \frac{a}{2}\left(\gamma^{i}-\gamma^{j}\right) \pm \sum_{1 \leq i<j \leq \ell} \frac{a}{2}\left(\gamma^{j}-\gamma^{i}\right)
$$




$$
=\frac{a}{2} \sum_{i \leq \ell<j}\left(\gamma^{j}-\gamma^{i}\right)=\frac{a}{2}\left[\left(\gamma^{1}+\cdots+\gamma^{\ell}\right)(r-\ell)-\left(\gamma^{\ell+1}+\cdots+\gamma^{r}\right) \ell\right]=-\frac{a}{2} d \chi(\gamma) .
$$

By Proposition 3.1.3 it follows that

$$
\Delta_{Q}(g)=\chi(g)^{a / 2}
$$

for all $g \in A \bar{N} \subset Q$. Now let $q \in Q$. Then $q e=g e$ for some (unique) $g \in A \bar{N}$, and hence $q=g k$, where $k \in Q \cap K$. It follows that

$$
\begin{aligned}
& \Delta_{Q}(q)=\Delta_{Q}(g k)=\Delta_{Q}(g) \Delta_{Q}(k)=\Delta_{Q}(g)=\chi(g)^{a / 2} \\
& \quad=\Delta(g e)^{(\ell-r) a / 2} \bar{\Delta}_{r-\ell}(g e)^{r a / 2}=\Delta(q e)^{(\ell-r) a / 2} \bar{\Delta}_{r-\ell}(q e)^{r a / 2} .
\end{aligned}
$$

Corollary 3.2.5 The Jacobian of $g \in G$, for the action (3.2.30) at $u_{\ell} \in \Pi_{\ell}$ is

$$
\rho_{\Pi_{\ell}}(g)=\Delta\left(g^{-1} e\right)^{(r-\ell) a / 2} \bar{\Delta}_{r-\ell}\left(g^{-1} e\right)^{-r a / 2} .
$$

Proof: Write $g=k q$ with $k \in K, q \in Q$. Then $g^{-1} e=q^{-1} k^{-1} e=q^{-1} e$ and hence

$$
\begin{aligned}
& \rho_{\Pi_{\ell}}(g)=\rho_{\Pi_{\ell}}(k q)=\left(\delta_{q} \rho_{\Pi_{\ell}}\right)(k)=\frac{\Delta_{Q}(q)}{\Delta_{G}(q)} \rho_{\Pi_{\ell}}(k)= \\
& \Delta_{Q}(q)=\Delta_{Q}^{-1}\left(q^{-1}\right)=\Delta\left(q^{-1} e\right)^{(r-\ell) a / 2} \bar{\Delta}_{r-\ell}\left(q^{-1} e\right)^{-r a / 2} .
\end{aligned}
$$

Theorem 3.2.6 For $0 \leq \ell<r$, define the character

$$
\chi_{\partial_{\ell} \Omega}(g):=\Delta(g e)^{\ell a / 2}
$$

on $G$. Then the (well-defined) quotient measure

$$
\mu_{\partial_{\ell} \Omega}:=\chi_{\partial_{\ell} \Omega} \cdot \mu_{G} / \mu_{P}
$$

on $G / P \approx \partial_{\ell} \Omega$ is relatively $G$-invariant with multiplier $\chi_{\partial_{\ell} \Omega}$ and has a decomposition

$$
\int_{\partial_{\ell} \Omega} d \mu_{\partial_{\ell} \Omega} f=\int_{\Pi_{\ell}} d u \int_{\Omega_{1}(u)} d \mu_{\Omega_{1}(u)}(\xi) \Delta(\xi+e-u)^{r a / 2} f(\xi)
$$

for all $f \in \mathcal{C}_{c}\left(\partial_{\ell} \Omega\right)$. Here du denotes the $K$-invariant measure on $\Pi_{\ell}$, and

$$
d \mu_{\Omega_{1}(u)}(\xi)=d \mu_{X_{1}(u)}(\xi) \Delta(\xi+e-u)^{-1-a(\ell-1) / 2}
$$

is the invariant measure on $\Omega_{1}(u)$.

Proof: For every $q \in Q$, the restriction $\stackrel{\vee}{q}:=\left.q\right|_{X_{1}\left(u_{\ell}\right)}$ belongs to $G L\left(\Omega_{1}\left(u_{\ell}\right)\right)$ and we may identify

$$
Q / P \ni q P \stackrel{\gtrless}{\rightleftarrows} q u_{\ell}=\stackrel{\vee}{q} u_{\ell} \in \Omega_{1}\left(u_{\ell}\right)
$$


in an equivariant way. It follows that $Q / P$ carries a $Q$-invariant measure

$$
d\left(\frac{\mu_{Q}}{\mu_{P}}\right)(q P)=d \mu_{\Omega_{1}\left(u_{\ell}\right)}\left(q u_{\ell}\right),
$$

and [Bou63, p.59, Corollaire 2] implies

$$
\Delta_{P}(p)=\Delta_{Q}(p) \quad \forall p \in P .
$$

Since $p e=p\left(u_{\ell}+e-u_{\ell}\right)=p u_{\ell}+p\left(e-u_{\ell}\right)=u_{\ell}+p\left(e-u_{\ell}\right)$, we have $\bar{\Delta}_{r-\ell}(p e)=$ $\bar{\Delta}_{r-\ell}\left(u_{\ell}+p\left(e-u_{\ell}\right)\right)=\Delta\left(u_{\ell}+p\left(e-u_{\ell}\right)\right)=\Delta(p e)$. Hence $P$ has the modulus function

$$
\Delta_{P}(p)=\Delta(p e)^{\ell a / 2} \quad \forall p \in P .
$$

Since $G$ is unimodular, Proposition 3.1.1 (ii) shows that $\mu_{\partial_{\ell} \Omega}$ is a well-defined $K$-invariant measure on $G / P \approx \partial_{\ell} \Omega$ which is relatively $G$-invariant with multiplier $\chi_{\partial_{\ell} \Omega}$. For $q \in Q$ we have

$$
\Delta(q e)=\Delta\left(q^{*} e\right)=\Delta_{\ell}\left(q^{*} e\right) \bar{\Delta}_{r-\ell}(q e)
$$

and

$$
\Delta_{\ell}\left(q^{*} e\right)=\Delta\left(q u_{\ell}+e-u_{\ell}\right)
$$

With (3.2.38), this implies

$$
\frac{\chi_{\partial_{\ell} \Omega}(q)}{\Delta_{Q}(q)}=\left[\frac{\Delta(q e)}{\bar{\Delta}_{r-\ell}(q e)}\right]^{r a / 2}=\Delta\left(q u_{\ell}+e-u_{\ell}\right)^{r a / 2} .
$$

Applying Proposition 3.1.1, we obtain

$$
\int_{\partial_{\ell} \Omega} d \mu_{\partial_{\ell} \Omega} f=\int_{K} d k \int_{\Omega_{1}\left(u_{\ell}\right)} d \mu_{\Omega_{1}\left(u_{\ell}\right)}(x) \Delta\left(x+e-u_{\ell}\right)^{r a / 2} f(k x)
$$

for all $f \in \mathcal{C}_{c}\left(\partial_{\ell} \Omega\right)$. Putting $u=k u_{\ell} \in \Pi_{\ell}$ and $\xi=k x \in \Omega_{1}(u)$, the assertion follows.

\subsection{Boundary orbits of symmetric Siegel domains}

For an irreducible hermitian Jordan triple $Z$, with maximal tripotent $e$, let $Z=U \oplus V$ be the Peirce decomposition

$$
U=Z_{1}(e), V=Z_{1 / 2}(e)
$$

with respect to $e$. Put $X:=\left\{x \in U: x^{*}=x\right\}$ and define $\tau: Z \rightarrow X$ by

$$
\tau(u, v):=\frac{u+u^{*}}{2}-\Phi(v, v),
$$

where $\Phi\left(v_{1}, v_{2}\right):=\left\{v_{1} v_{2}^{*} e\right\}$ for all $v_{1}, v_{2} \in V$. Then $\tau(e)=e$, and

$$
D:=\{(u, v) \in Z: \tau(u, v) \in \Omega\}
$$

is a symmetric Siegel domain. Let $\operatorname{Aut}(D)$ be the holomorphic automorphism group of $D$. 
For $(a, b) \in i X \times V$, the "quasi-translations"

$$
t_{a, b}(u, v):=(u+a+\Phi(b, b)+2 \Phi(v, b), v+b)
$$

belong to $\operatorname{Aut}(D)$, since $\tau\left(t_{a, b}(u, v)\right)=\tau(u, v)$. Moreover, the relation

$$
t_{a_{1}, b_{1}} t_{a_{2}, b_{2}}=t_{a_{1}+a_{2}+\Phi\left(b_{2}, b_{1}\right)-\Phi\left(b_{1}, b_{2}\right), b_{1}+b_{2}}
$$

implies that

$$
\Sigma:=\left\{t_{a, b}: a \in i X, b \in V\right\}
$$

is a nilpotent (hence unimodular) subgroup of $A u t(D)$, with Haar measure

$$
d \mu_{\Sigma}\left(t_{a, b}\right)=d \mu_{i X}(a) d \mu_{V}(b)
$$

given by the product of the Lebesgue measures on $i X$ and $V$. The group $G L(D):=\operatorname{Aut}(D) \cap$ $G L(Z)$ consists of all linear transformations

$$
h(u, v)=\left(h_{1} u, h_{2} v\right)
$$

on $Z=U \oplus V$ such that $h_{1} \in G L(\Omega) \subset G L(U)$ (by complexification), and $h_{2} \in G L(V)$ satisfies

$$
\Phi\left(h_{2} v, h_{2} b\right)=h_{1} \Phi(v, b)
$$

for all $v, b \in V$. Therefore

$$
\tau(h z)=h_{1} \tau(z)
$$

for all $z \in D$, and the homomorphism

$$
G L(D) \ni h \mapsto h_{1} \in G L(\Omega)
$$

has a compact kernel. Using [Bou63, p.61, Corollaire] it follows that

$$
\Delta_{G L(D)}(h)=\Delta_{G L(\Omega)}\left(h_{1}\right)=1
$$

for all $h \in G L(D)$. For $a \in i X, b \in V$ and $h=h_{1} \times h_{2} \in G L(D)$, we have

$$
h t_{a, b} h^{-1}=t_{h_{1} a, h_{2} b} .
$$

Thus we may consider the semidirect product

$$
\operatorname{Aff}(D)=\Sigma \cdot G L(D) \subset \operatorname{Aut}(D)
$$

consisting of all affine transformations of $D$. The symmetry $s_{e}$ of $D$ around $e$ satisfies

$$
s_{e} h^{-1} s_{e}=h^{*} \in G L(D)
$$

for all $h \in G L(D)$. This can also be written as

$$
\left(s_{e} h^{-1} s_{e}\right)^{\prime}(0)=h^{*} \in G L(Z) .
$$


Lemma 3.3.1 For $h \in G L(D)$ the automorphism $i_{h}(t):=h^{-1} t h$ of $\Sigma$ has the modulus

$$
\Delta_{\Sigma}\left(i_{h}\right)=\Delta(h e)^{-n / r}
$$

where $\Delta$ is the Jordan determinant of $X$.

Proof: The commuting diagram

$$
\begin{array}{ccccc}
t_{a, b} & \in & \Sigma & \stackrel{i_{h}^{-1}}{\longrightarrow} & \Sigma \\
\uparrow & & \approx \uparrow & & \uparrow \approx \\
(a, b) & \in & i X \times V & \underset{h_{1} \times h_{2}}{\longrightarrow} & i X \times V
\end{array}
$$

implies

$$
\begin{gathered}
\Delta_{\Sigma}\left(i_{h}^{-1}\right)=\Delta_{i X \times V}\left(h_{1} \times h_{2}\right)=\left|\operatorname{Det}_{X} h_{1}\right| \cdot\left|\operatorname{Det}_{V} h_{2}\right|^{2} \\
=\left|\operatorname{Det}_{X} h_{1}\right| \cdot \frac{\left|\operatorname{Det}_{Z} h\right|^{2}}{\left|\operatorname{Det}_{U} h_{1}\right|^{2}}=\frac{\left|\operatorname{Det}_{Z} h\right|^{2}}{\left|\operatorname{Det}_{X} h_{1}\right|} .
\end{gathered}
$$

Since $h_{1} \in G L(\Omega)$, we have

$$
\left|\operatorname{Det}_{X} h_{1}\right|=\Delta(h e)^{n_{1} / r}
$$

where $n_{1}=\operatorname{dim} U$. On the other hand, every $g \in A u t(D)$ satisfies

$$
\left|\operatorname{Det}_{Z} g^{\prime}(e)\right|^{2}=\Delta(\tau(g(e)))^{p}
$$

since $\Delta(\tau(z, w))^{-p}$ is the Bergman kernel of $D$. Therefore

$$
\left|\operatorname{Det}_{Z} h\right|^{2}=\Delta(\tau(h e))^{p}=\Delta(h e)^{p} .
$$

Since $p=\frac{n_{1}+n}{r}$, it follows that

$$
\Delta_{\Sigma}\left(i_{h}^{-1}\right)=\frac{\Delta(h e)^{p}}{\Delta(h e)^{n_{1} / r}}=\Delta(h e)^{n / r}
$$

Corollary 3.3.2 Aff $(D)$ has the modulus function

$$
\Delta_{A f f(D)}(g)=\Delta(\tau(g(e)))^{-n / r} .
$$

Proof: Write $g \in A f f(D)$ as $g=t h$, with $t \in \Sigma$ and $h \in G L(D)$. Since $\tau(g(e))=h_{1} e$, [Bou63, p.61, Corollaire], (3.3.7) and Lemma 3.3.1 imply

$$
\Delta_{A f f(D)}(g)=\Delta_{\Sigma}\left(i_{h}\right) \Delta_{G L(D)}(h)=\Delta_{\Sigma}\left(i_{h}\right)=\Delta\left(h_{1} e\right)^{-n / r}=\Delta(\tau(g(e)))^{-n / r}
$$

We now turn to the Iwasawa decomposition

$$
\operatorname{Aut}(D)=N A K
$$


of the (semi-simple) Lie group $A u t(D)$. We have

$$
N A=\Sigma \cdot(N A \cap G L(D)) \subset \operatorname{Aff}(D)
$$

(semi-direct product), and

$$
N A \cap G L(D) \ni h \mapsto h_{1} \in N_{\Omega} A_{\Omega}
$$

is a group isomorphism.

Proposition 3.3.3 NA has the modulus function

$$
\Delta_{N A}(g)=\Delta_{-2 \rho}(\tau(g(e)))
$$

where $2 \rho_{k}=a(k-1)+1+b$.

Proof: Write $g=t h \in N A$, with $t \in \Sigma$ and $h \in N A \cap G L(D)$. Then $\tau(g(e))=\tau(h e)=h_{1} e$ and [Bou63, p.61, Corollaire] and Lemma 3.3.1 and Proposition 3.2.3 imply

$$
\begin{aligned}
\Delta_{N A}(t h) & =\Delta_{\Sigma}\left(i_{h}\right) \Delta_{N A \cap G L(D)}(h) \\
& =\Delta\left(h_{1} e\right)^{-n / r} \Delta_{-2 \rho_{\Omega}}\left(h_{1} e\right)=\Delta_{-\frac{n}{r}-2 \rho_{\Omega}}\left(h_{1} e\right)
\end{aligned}
$$

with $\frac{n}{r}+2\left(\rho_{\Omega}\right)_{k}=1+\frac{a}{2}(r-1)+b+\frac{a}{2}(2 k-r-1)=a(k-1)+1+b$.

Fix $0 \leq \ell<r$ and put $u_{\ell}=e_{1}+\cdots+e_{\ell}$. Then

$$
\partial_{\ell} D=\Sigma \cdot \partial_{\ell} \Omega=\left\{t x: t \in \Sigma, x \in \partial_{\ell} \Omega\right\}=\operatorname{Aff}(D)\left(u_{\ell}\right)
$$

is an $\operatorname{Aff}(D)$-orbit, i.e., we may identify

$$
\text { Aff }(D) / P \ni g P \mapsto g\left(u_{\ell}\right) \in \partial_{\ell} D,
$$

where

$$
P:=\left\{g \in \operatorname{Aff}(D): g\left(u_{\ell}\right)=u_{\ell}\right\}
$$

is a closed subgroup of $\operatorname{Aff}(D)$, with Lie algebra

$$
\wp:=\left\{\gamma \in \operatorname{aff}(D): \gamma\left(u_{\ell}\right)=0\right\} .
$$

For $u \in \Pi_{\ell}$, let

$$
D_{1}(u)=\Sigma_{1}(u) \cdot \Omega_{1}(u) \subset Z_{0}(e-u)
$$

denote the Siegel domain associated with $u \in Z_{0}(e-u)$. Here

$$
\Sigma_{1}(u):=\left\{t_{a, b} \in \Sigma: a \in i X_{1}(u), b \in V_{1 / 2}(u)\right\}
$$

is a closed subgroup of $\Sigma$. One can show [U85], that

$$
\partial_{\ell} D=\bigcup_{u \in \Pi_{\ell}} D_{1}(u) \quad \text { (disjoint union) . }
$$


Theorem 3.3.4 Let $\mu_{\partial_{\ell} \Omega}$ denote the Lassalle measure on $\partial_{\ell} \Omega$ (cf. Theorem 3.2.6). Then the product measure

$$
d \mu_{\partial_{\ell} D}(t x):=d \mu_{\Sigma}(t) \cdot d \mu_{\partial_{\ell} \Omega}(x)
$$

on $\partial_{\ell} D$ is relatively invariant under $\operatorname{Aff}(D)$, with multiplier

$$
\chi_{\partial_{\ell} D}(g):=\Delta(\tau(g(e)))^{\alpha_{\ell}}
$$

for all $g \in \operatorname{Aff}(D)$. Here $\alpha_{\ell}=\frac{n}{r}+\ell \frac{a}{2}$. Moreover there is a decomposition

$$
\int_{\partial_{\ell} D} d \mu_{\partial_{\ell} D} f=\int_{\Pi_{\ell}} d u \int_{\Sigma / \Sigma_{1}(u)} d t \int_{D_{1}(u)} d \mu_{D_{1}(u)}(\omega) \Delta(\tau(\omega+e-u))^{\alpha_{\ell}} f(t \omega)
$$

for all $f \in \mathcal{C}_{c}\left(\partial_{\ell} D\right)$, where

$$
d \mu_{D_{1}(u)}(\omega)=d \mu_{Z_{0}(u-e)}(\omega) \cdot \Delta(\tau(\omega+e-u))^{-(2+a(\ell-1)+b)}
$$

is the invariant measure on $D_{1}(u)$.

Proof: Write $g \in \operatorname{Aff}(D)$ as $g=t_{0} h$, where $t_{0} \in \Sigma$ and $h=h_{1} \times h_{2} \in G L(D)$. Then we have

$$
\begin{aligned}
& \int_{\partial_{\ell} D} d\left(g_{*}^{-1} \mu_{\partial_{\ell} D}\right) f=\int_{\partial_{\ell} D} d \mu_{\partial_{\ell} D} f \circ g^{-1}=\int_{\Sigma} d \mu_{\Sigma}(t) \int_{\partial_{\ell} \Omega} d \mu_{\partial_{\ell} \Omega}(x) f\left(h^{-1} t_{0}^{-1} t x\right)= \\
& \int_{\Sigma} d \mu_{\Sigma}(t) \int_{\partial_{\ell} \Omega} d \mu_{\partial_{\ell} \Omega}(x) f\left(h^{-1} t x\right)=\int_{\Sigma} d \mu_{\Sigma}(t) \int_{\partial_{\ell} \Omega} d \mu_{\partial_{\ell} \Omega}(x) f\left(i_{h}(t) h_{1}^{-1} x\right)= \\
& \Delta\left(h_{1} e\right)^{\ell a / 2} \int_{\Sigma} d \mu_{\Sigma}(t) \int_{\partial_{\ell} \Omega} d \mu_{\partial_{\ell} \Omega}(x) f\left(i_{h}(t) x\right)= \\
& \Delta\left(h_{1} e\right)^{\ell a / 2} \Delta_{\Sigma}^{-1}\left(i_{h}\right) \int_{\Sigma} d \mu_{\Sigma}(t) \int_{\partial_{\ell} \Omega} d \mu_{\partial_{\ell} \Omega}(x) f(t x)= \\
& \Delta\left(h_{1} e\right)^{\ell a / 2} \Delta\left(h_{1} e\right)^{n / r} \int_{\partial_{\ell} D} d \mu_{\partial_{\ell} D} f=\Delta\left(h_{1} e\right)^{\alpha_{\ell}} \int_{\partial_{\ell} D} d \mu_{\partial_{\ell} D} f .
\end{aligned}
$$

Since $\tau(g(e))=\tau\left(t_{0}(h e)\right)=\tau(h e)=h_{1} e$, it follows that $\mu_{\partial_{\ell} D}$ is $\operatorname{Aff}(D)$-relatively invariant with multiplier given by (3.3.39). For every $u \in \Pi_{\ell}$, we have

$$
Z_{0}(u-e)=Z_{1 / 2}(u) \oplus Z_{1}(u)=\Sigma_{1}(u) \cdot X_{1}(u)
$$

where

$$
\Sigma_{1}(u):=\left\{t_{a, b}: a \in i X_{1}(u), b \in V_{1 / 2}(u)\right\} .
$$

Therefore the respective Lebesgue measures satisfy

$$
d \mu_{Z_{0}(u-e)}(t \xi)=d \mu_{\Sigma_{1}(u)}(t) d \mu_{X_{1}(u)}(\xi) .
$$

Similarly,

$$
D_{1}(u)=\Sigma_{1}(u) \cdot \Omega_{1}(u)
$$

and

$$
\begin{aligned}
& d \mu_{D_{1}(u)}(t \xi) \Delta(\tau(t \xi+e-u))^{\alpha_{\ell}}= \\
& \quad d \mu_{Z_{0}(u-e)}(t \xi) \Delta(\tau(t \xi+e-u))^{\alpha_{\ell}-(2+a(\ell-1)+b)}= \\
& \quad d \mu_{\Sigma_{1}(u)}(t) d \mu_{X_{1}(u)}(\xi) \Delta(\xi+e-u)^{-1+\frac{a}{2}(r-\ell+1)}= \\
& \quad d \mu_{\Sigma_{1}(u)}(t) d \mu_{\Omega_{1}(u)}(\xi) \Delta(\xi+e-u)^{a r / 2}
\end{aligned}
$$


since $\tau(t \xi+e-u)=\xi+e-u$. Therefore Theorem 3.2.6 yields

$$
\begin{aligned}
& \int_{\partial_{\ell} D} d \mu_{\partial_{\ell} D} f=\int_{\Sigma} d \mu_{\Sigma}(t) \int_{\partial_{\ell} \Omega} d \mu_{\partial_{\ell} \Omega}(x) f(t x)= \\
& \quad \int_{\Sigma} d \mu_{\Sigma}(t) \int_{\Pi_{\ell}} d u \int_{\Omega_{1}(u)} d \mu_{\Omega_{1}(u)}(\xi) \Delta(\xi+e-u)^{a r / 2} f(t \xi)= \\
& \quad \int_{\Pi_{\ell}} d u \int_{\Sigma / \Sigma_{1}(u)} d \dot{t} \int_{\Sigma_{1}(u)} d \mu_{\Sigma_{1}(u)}\left(t_{0}\right) \int_{\Omega_{1}(u)} d \mu_{\Omega_{1}(u)}(\xi) \Delta(\xi+e-u)^{a r / 2} f\left(t t_{0} \xi\right) \\
& \quad=\int_{\Pi_{\ell}} d u \int_{\Sigma / \Sigma_{1}(u)} d \dot{t} \int_{D_{1}(u)} d \mu_{D_{1}(u)}(\omega) \Delta(\tau(\omega+e-u))^{\alpha_{\ell}} f(t \omega)
\end{aligned}
$$

by putting $\omega=t_{0} \xi$.

\subsection{Boundary orbits of Cartan domains}

Let $Z$ be an irreducible hermitian Jordan triple of rank $r$, with open unit ball $B$. Then

$$
G:=\operatorname{Aut}(B)
$$

is a semi-simple Lie group, with Lie algebra

$$
\mathfrak{g}=\operatorname{aut}(B)
$$

consisting of all completely integrable holomorphic vector fields

$$
f(z) \frac{\partial}{\partial z}
$$

on $B$, under the commutator

$$
\left[f(z) \frac{\partial}{\partial z}, g(z) \frac{\partial}{\partial z}\right]=\left(f^{\prime}(z) g(z)-g^{\prime}(z) f(z)\right) \frac{\partial}{\partial z} .
$$

For $a \in Z$, we abbreviate

$$
a-\{\stackrel{*}{a}\}:=\left(a-\left\{z^{*} a\right\}\right) \frac{\partial}{\partial z} \in \mathfrak{g} .
$$

$G$ has a maximal compact subgroup

$$
K=\operatorname{Aut}(Z)=\{g \in G: g(0)=0\}
$$

consisting of linear transformations (in fact, Jordan triple automorphisms), and its Lie algebra

$$
\mathfrak{k}=\left\{\lambda z \frac{\partial}{\partial z}: \lambda \in \operatorname{aut}(Z)\right\}
$$

consists of all Jordan triple derivations $\lambda$ regarded as linear vector fields $\lambda z \frac{\partial}{\partial z}$. Since, by (3.4.4), for all $\lambda, \mu \in$ aut $(Z)$

$$
\left[\lambda z \frac{\partial}{\partial z}, \mu z \frac{\partial}{\partial z}\right]=[\lambda, \mu] z \frac{\partial}{\partial z}
$$


we will identify $\lambda \in \operatorname{aut}(Z)$ with the corresponding linear vector field $\lambda z \frac{\partial}{\partial z}$. Then

$$
[\lambda, a-\{\stackrel{*}{a}\}]=\lambda a-\left\{(\lambda a)^{*}\right\}
$$

for all $\lambda \in \mathfrak{k}$ and $a \in Z$. Fix a frame $\left\{e_{1}, \ldots, e_{r}\right\}$ of tripotents in $Z$, and consider the associated Peirce decomposition

$$
Z=\sum_{0 \leq i \leq j \leq r}^{\oplus} Z_{i j}
$$

With respect to the Cartan subspace

$$
\mathfrak{a}:=\left\langle e_{k}-\left\{e_{k}^{*}\right\}: 1 \leq k \leq r\right\rangle \quad(\text { real span }),
$$

we obtain a real root decomposition

$$
\mathfrak{g}=\mathfrak{m} \oplus \mathfrak{a} \oplus \sum_{1 \leq i<j \leq r}\left(\mathfrak{g}_{j-i}^{+} \oplus \mathfrak{g}_{j-i}^{-}\right) \oplus \sum_{0 \leq i \leq j \leq r}\left(\mathfrak{g}_{j+i}^{+} \oplus \mathfrak{g}_{j+i}^{-}\right)
$$

Here

$$
\mathfrak{m}:=\left\{\delta \in \operatorname{aut}(Z): \delta e_{k}=0 \quad \forall 1 \leq k \leq r\right\}
$$

and, for $\kappa= \pm$ and $\varepsilon= \pm 1, \quad \mathfrak{g}_{j-\varepsilon i}^{\kappa}$ consists of all vector fields of the form

$$
\gamma=a-\{\stackrel{*}{a}\}+\kappa \#_{i j}\left(e_{j} \square \stackrel{*}{a}-a \square \stackrel{*}{e}_{j}\right)
$$

with $a \in Z_{i j}$ and $\stackrel{*}{a}=\varepsilon a$ (if $i>0$ ). Here

$$
\#_{i j}:= \begin{cases}2 & i \neq j \\ 1 & i=j .\end{cases}
$$

For the commutator, one obtains

$$
\left[e_{k}-\{\stackrel{*}{e} k\}, \gamma\right]=2\left(e_{k} \square \stackrel{*}{a}-a \square \stackrel{*}{e_{k}}\right)+\kappa \#_{i, j}(b-\{\stackrel{*}{b}\}),
$$

where $b=\left\{a \stackrel{*}{e_{j}} e_{k}\right\}-\left\{e_{j} \stackrel{*}{a} e_{k}\right\}$. We claim that for all $1 \leq k \leq r$

$$
\#_{i, j}\left(\left\{a \stackrel{*}{e_{j}} e_{k}\right\}-\left\{e_{j} \stackrel{*}{a} e_{k}\right\}\right)=\left(\delta_{k}^{j}-\varepsilon \delta_{k}^{i}\right) a
$$

and

$$
2\left(e_{k} \square \stackrel{*}{a}-a \square \stackrel{*}{e_{k}}\right)=\left(\delta_{k}^{j}-\varepsilon \delta_{k}^{i}\right) \#_{i, j}\left(e_{j} \square \stackrel{*}{a}-a \square \stackrel{*}{e_{j}}\right) .
$$

For $k \notin\{i, j\}$, this is trivial. Now assume $k \in\{i, j\}$. Assume first $0 \leq i<j \leq r$. Then

$$
2\left(\left\{a \stackrel{*}{e_{j}} e_{j}\right\}-\left\{e_{j} \stackrel{*}{a} e_{j}\right\}\right)=2\left\{a \stackrel{*}{e_{j}} e_{j}\right\}=a,
$$

(3.4.17) is trivial for $k=j$, and in case $i \geq 1$

$$
2\left(\left\{a \stackrel{*}{e_{j}} e_{i}\right\}-\left\{e_{j} \stackrel{*}{a} e_{i}\right\}\right)=-2\left\{e_{j} \stackrel{*}{a} e_{i}\right\}=-\stackrel{*}{a}=-\varepsilon a
$$

and

$$
2\left(e_{i} \square \stackrel{*}{a}-a \square \stackrel{*}{e_{i}}\right)=2\left(\stackrel{*}{a} \square \stackrel{*}{e}_{j}-e_{j} \square\left(\stackrel{*}{a}^{*}\right)=2 \varepsilon\left(a \square \stackrel{*}{e_{j}}-e_{j} \square \stackrel{*}{a}\right)\right.
$$


Thus (3.4.16) and (3.4.17) hold if $i<j$. Now let $1 \leq i=j \leq r$. Then $\varepsilon=-1$ and hence

$$
\left\{a \stackrel{*}{e_{j}} e_{j}\right\}-\left\{e_{j} \stackrel{*}{a} e_{j}\right\}=a-\stackrel{*}{a}=(1-\varepsilon) a
$$

and (3.4.17) is trivial for $k=j$ since $1-\varepsilon=2$. Thus (3.4.16) and (3.4.17) hold for $i=j$ also. It follows that

$$
\left[e_{k}-\left\{e_{k}^{*}\right\}, \gamma\right]=\kappa\left(\delta_{k}^{j}-\varepsilon \delta_{k}^{i}\right) \gamma
$$

showing that $\mathfrak{g}_{j-\varepsilon i}^{\kappa}$ is the root space belonging to the root $\kappa\left(\delta^{j}-\varepsilon \delta^{i}\right)$ defined by

$$
\kappa\left(\delta^{j}-\varepsilon \delta^{i}\right)\left(e_{k}-\left\{e_{k}^{*}\right\}\right):=\kappa\left(\delta_{k}^{j}-\varepsilon \delta_{k}^{i}\right)
$$

Lemma 3.4.1 Let $G=$ Aut $(B)$ and, for some $o \in \bar{B} \subset Z$, put

$$
P:=\{p \in G: p(o)=o\} .
$$

Then

$$
\chi(p):=\left|\operatorname{Det}_{Z} p^{\prime}(o)\right|
$$

is a character of $P$, with differential

$$
d \chi(\gamma)=\operatorname{Re} \operatorname{tr}_{Z} \gamma^{\prime}(o) \quad \forall \gamma \in \wp
$$

Proof: The group $G$ acts on the compact dual space $M$ of $B$ which contains $Z$ as an open dense subset. Since $o \in Z$ it follows that $Z \cap p^{-1}(Z)$ is an open neighborhood of $o$ for each $p \in P$. Therefore $p^{\prime}(o) \in G L(Z)$ is well-defined, and

$$
\left(p_{1} p_{2}\right)^{\prime}(o)=p_{1}^{\prime}(o) p_{2}^{\prime}(o)
$$

for all $p_{1}, p_{2} \in P$ since $p_{2}(o)=o$. Thus (3.4.25) defines a character of $P$. Now let $\gamma \in \wp$. Then $g_{t}(z):=\exp _{B}(t \gamma)(z)$ satisfies

$$
\frac{\partial g_{t}(z)}{\partial t}=\gamma\left(g_{t}(z)\right)
$$

and hence

$$
\frac{\partial g_{t}^{\prime}(o)}{\partial t}=\left(\gamma \circ g_{t}\right)^{\prime}(o)=\gamma^{\prime}\left(g_{t}(o)\right) g_{t}^{\prime}(o)=\gamma^{\prime}(o) g_{t}^{\prime}(o)
$$

It follows that $\left(\exp _{B} t \gamma\right)^{\prime}(o)=\exp _{Z} t \gamma^{\prime}(o)$ and therefore

$$
\begin{aligned}
& \chi\left(\exp _{B}(t \gamma)\right)=\left|\operatorname{Det}_{Z} \exp _{B}(t \gamma)^{\prime}(o)\right|=\left|\operatorname{Det} \exp _{Z} t \gamma^{\prime}(o)\right| \\
& =\left|\exp \operatorname{tr}_{Z} t \gamma^{\prime}(o)\right|=\exp \left(\operatorname{Re} \operatorname{tr}_{Z} t \gamma^{\prime}(o)\right) .
\end{aligned}
$$

Hence

$$
d \chi(\gamma)=\left.\frac{d}{d t}\right|_{t=0} \chi\left(\exp _{B}(t \gamma)\right)=\operatorname{Re}_{\operatorname{tr}} \gamma^{\prime}(o) .
$$

Fix $0 \leq \ell<r$ and put $u_{\ell}-e=-e_{\ell+1}-\cdots-e_{r}$. Consider the $G$-orbit

$$
\partial_{\ell} B:=G\left(u_{\ell}-e\right) \subset \partial B
$$


Then there is a diffeomorphism

$$
G / P \ni g P \mapsto g\left(u_{\ell}-e\right) \in \partial_{\ell} B,
$$

where

$$
P:=\left\{p \in G: p\left(u_{\ell}-e\right)=u_{\ell}-e\right\} \ni g P \mapsto g\left(u_{\ell}-e\right) \in \partial_{\ell} B
$$

is a closed subgroup of $G$, with Lie algebra

$$
\wp:=\left\{\gamma \in \mathfrak{g}: \gamma\left(u_{\ell}-e\right)=0\right\} .
$$

Let $S_{r-\ell}$ denote the compact manifold of all tripotents of rank $r-\ell$ in $Z$. For every $v \in S_{r-\ell}$, the unit ball

$$
B_{0}(v) \subset Z_{0}(v)
$$

is a symmetric ball of rank $\ell$, and there is a fibration

$$
\partial_{\ell} B=\bigcup_{v \in S_{r-\ell}} v+B_{0}(v)
$$

Since $G$ permutes the fibres of (3.4.37), there exists an action $g \mapsto \widetilde{g}$ of $G$ on $S_{r-\ell}$ satisfying

$$
g\left(v+B_{0}(v)\right)=\widetilde{g}(v)+B_{0}(\widetilde{g}(v))
$$

for all $v \in S_{r-\ell}$. This action is transitive, and hence there exists a diffeomorphism

$$
G / Q \ni g Q \mapsto \widetilde{g}\left(u_{\ell}-e\right) \in S_{r-\ell}
$$

where

$$
\begin{aligned}
Q & :=\left\{q \in G: \widetilde{q}\left(u_{\ell}-e\right)=u_{\ell}-e\right\} \\
& =\left\{q \in G: q\left(u_{\ell}-e\right) \in u_{\ell}-e+B_{0}\left(u_{\ell}-e\right)\right\}
\end{aligned}
$$

is a closed subgroup containing $P$, with Lie algebra

$$
\mathfrak{q}:=\left\{\gamma \in \mathfrak{g}: \gamma\left(u_{\ell}-e\right) \in Z_{0}\left(u_{\ell}-e\right)\right\} .
$$

If $q \in Q \cap K$ then $q \in S_{r-\ell} \cap\left(u_{\ell}-e+B_{0}\left(u_{\ell}-e\right)\right)=\left\{u_{\ell}-e\right\}$ and hence $q \in P$. Thus $Q \cap K=P \cap K$. Evaluating at $u_{\ell}-e$, we have

$$
\delta\left(u_{\ell}-e\right)=-\sum_{k>\ell} \delta e_{k}=0 \quad \text { for all } \quad \delta \in \mathfrak{m},
$$

whereas

$$
e_{k}-\left\{\left(u_{\ell}-e\right) e_{k}^{*}\left(u_{\ell}-e\right)\right\}=\left\{\begin{array}{cc}
e_{k} & k \leq \ell \\
0 & k>\ell
\end{array}\right.
$$

and

$$
\begin{gathered}
a-\left\{\left(u_{\ell}-e\right) \stackrel{*}{a}\left(u_{\ell}-e\right)\right\}+\kappa \#_{i, j}\left(\left\{e_{j} \stackrel{*}{a}\left(u_{\ell}-e\right)\right\}-\left\{a \stackrel{*}{e}_{j}\left(u_{\ell}-e\right)\right\}=\right. \\
\qquad \begin{cases}a-\stackrel{*}{a}+2 \kappa\left(-\frac{a^{*}}{2}+\frac{a}{2}\right)=(1+\kappa)(1-\varepsilon) a & \ell<i<j \\
a-\stackrel{*}{a}+\kappa(-\stackrel{*}{a}+a)=(1+\kappa)(1-\varepsilon) a & \ell<i=j \\
a+2 \kappa\left(\frac{a}{2}\right)=(1+\kappa) a & 0 \leq i \leq \ell<j \\
a & 0 \leq i \leq j \leq \ell\end{cases}
\end{gathered}
$$


for all $a \in Z_{i j}$ with $\stackrel{*}{a}=\varepsilon a$ (if $\left.i>0\right)$. It follows that

$$
\begin{gathered}
\wp=\mathfrak{m} \oplus\left\langle e_{k}-\left\{e_{k}^{*}\right\}: k>\ell\right\rangle \oplus \sum_{i<j>\ell} \mathfrak{g}_{j-i}^{-} \oplus \sum_{\ell<i<j} \mathfrak{g}_{j-i}^{+} \oplus \sum_{0 \leq i \leq j>\ell} \mathfrak{g}_{j+i}^{+}, \\
\mathfrak{g}=\wp \oplus\left\langle e_{k}-\left\{e_{k}^{*}\right\}: k \leq \ell\right\rangle \oplus \sum_{1 \leq i<j \leq \ell}\left(\mathfrak{g}_{j-i}^{+} \oplus \mathfrak{g}_{j-i}^{-}\right) \oplus \sum_{0 \leq i \leq j \leq \ell}\left(\mathfrak{g}_{j+i}^{+} \oplus \mathfrak{g}_{j+i}^{-}\right) .
\end{gathered}
$$

This implies $q \supset \mathfrak{a} \oplus \overline{\mathfrak{n}}$ and $Q \supset A \bar{N}$. Therefore

$$
Q / Q \cap K \approx Q(0)=A \bar{N}(0)=B=G(0),
$$

showing that $G=Q K$.

Lemma 3.4.2 $P$ has the modulus function $\Delta_{P}(p)=\left|\operatorname{Det}_{Z} p^{\prime}\left(u_{\ell}-e\right)\right|^{2 \alpha_{\ell} / p}$.

Proof: Since $P$ fixes $u_{\ell}-e$, Lemma 3.4.1 shows that $\chi(p):=\left|\operatorname{Det}_{Z} p^{\prime}\left(u_{\ell}-e\right)\right|$ defines a character of $P$, with differential

$$
d \chi(\gamma)=\operatorname{Re} \operatorname{tr}_{Z} \gamma^{\prime}\left(u_{\ell}-e\right)
$$

for all $\gamma \in \wp$. Now let

$$
\gamma=a-\{\stackrel{*}{a}\}+\kappa \#_{i j}\left(e_{j} \square \stackrel{*}{a}-a \square \stackrel{*}{e_{j}}\right) \in \wp
$$

where $a \in Z_{i j}$. Then

$$
\gamma^{\prime}\left(u_{\ell}-e\right)=-2\left(u_{\ell}-e\right) \square \stackrel{*}{a}+\kappa \#_{i j}\left(e_{j} \square \stackrel{*}{a}-a \square \stackrel{*}{e_{j}}\right)
$$

satisfies

$$
\frac{2}{p} \operatorname{Re} \operatorname{tr}_{Z} \gamma^{\prime}\left(u_{\ell}-e\right)=-2\left(u_{\ell}-e \mid a\right)+\kappa \#_{i j}\left(\left(e_{j} \mid a\right)-\left(a \mid e_{j}\right)\right)
$$

since $\operatorname{tr}_{Z} u \square v^{*}=\frac{p}{2}(u \mid v)$ for all $u, v \in Z$. This vanishes if $i<j$. For $i=j>\ell$, we have $\kappa=-1$ and

$$
\frac{2}{p} \operatorname{Re} \operatorname{tr}_{Z} \gamma^{\prime}\left(u_{\ell}-e\right)=2\left(e_{j} \mid a\right)-\left(\left(e_{j} \mid a\right)-\left(a \mid e_{j}\right)\right)=\left(e_{j} \mid a\right)+\left(a \mid e_{j}\right)=0
$$

since $a^{*}=-a$. Now let $\gamma=\sum_{k} \gamma^{k}\left(e_{k}-\left\{e_{k}^{*}\right\}\right) \in \mathfrak{a}$. Then

$$
\gamma^{\prime}\left(u_{\ell}-e\right)=-2 \sum_{k} \gamma^{k}\left(u_{\ell}-e\right) \square \stackrel{*}{e_{k}}
$$

satisfies

$$
\frac{2}{p} \operatorname{Re}_{\operatorname{tr}} \gamma^{\prime}\left(u_{\ell}-e\right)=-2 \sum_{k} \gamma^{k}\left(u_{\ell}-e \mid e_{k}\right)=2 \sum_{k>\ell} \gamma^{k}
$$

Since $\operatorname{tr} a d_{\wp} \gamma=\operatorname{tr} a d_{\mathfrak{q}} \gamma=-2 \alpha_{\ell} \sum_{k>\ell} \gamma^{k}$, Proposition 3.1.3 implies $\Delta_{P}(p)=\chi(p)^{2 \alpha_{\ell} / p}$ provided $P / P \cap K$ is connected. Since $P \cap K=Q \cap K$, and $Q / Q \cap K \approx B$ and $Q / P \approx B_{0}\left(u_{\ell}-e\right)$ are simply connected, this follows from the exact homotopy sequence applied to the fibration

$$
P / P \cap K \rightarrow Q / P \cap K \rightarrow Q / P \text {. }
$$


For $g \in G, v \in S_{r-\ell}$ and $\zeta \in B_{0}(v)$, we write

$$
g(v+\zeta)=\widetilde{g}(v)+g_{v}(\zeta),
$$

where

$$
g_{v}: B_{0}(v) \rightarrow B_{0}(\widetilde{g}(v))
$$

is biholomorphic. Specializing to $v=u_{\ell}-e$, we obtain a group homomorphism

$$
Q \ni q \mapsto q_{u_{\ell}-e} \in \text { Aut } B_{0}\left(u_{\ell}-e\right)
$$

determined by

$$
q\left(u_{\ell}-e+\zeta\right)=u_{\ell}-e+q_{u_{\ell}-e}(\zeta)
$$

for all $\zeta \in B_{0}\left(u_{\ell}-e\right)$.

Proposition 3.4.3 $Q$ has the modulus function

$$
\Delta_{Q}(q)=\left[\frac{\left|\operatorname{Det}_{Z} q^{\prime}\left(u_{\ell}-e\right)^{2 / p}\right|}{\Delta\left(q_{u_{\ell}-e}(0), q_{u_{\ell}-e}(0)\right)}\right]^{\alpha_{\ell}} .
$$

Proof: The identification

$$
Q / P \ni q P \mapsto q_{u_{\ell}-e}(0) \in B_{0}\left(u_{\ell}-e\right)
$$

yields a $Q$-invariant measure

$$
d\left(\frac{\mu_{Q}}{\mu_{P}}\right)(q P)=d \mu_{B_{0}\left(u_{\ell}-e\right)}\left(q_{u_{\ell}-e}(0)\right) .
$$

By [Bou63, p.59, Corollaire 2], this implies

$$
\Delta_{Q}(p)=\Delta_{P}(p) \quad \forall p \in P .
$$

The Lie algebra aut $B_{0}\left(u_{\ell}-e\right)$ consists of all vector fields

$$
X=\left(\left\{a b^{*} \zeta\right\}-\left\{b a^{*} \zeta\right\}+c-\left\{\zeta c^{*} \zeta\right\}\right) \frac{\partial}{\partial \zeta}
$$

on $B_{0}\left(u_{\ell}-e\right)$, where $a, b, c \in Z_{0}\left(u_{\ell}-e\right)$ are arbitrary. Moreover, the vector field

$$
Y:=\left(\left\{a b^{*} z\right\}-\left\{b a^{*} z\right\}+c-\left\{z c^{*} z\right\}\right) \frac{\partial}{\partial z}
$$

on $B$ belongs to $\mathfrak{q}$, and there is a Lie algebra homomorphism

$$
\pi: \text { aut } B_{0}\left(u_{\ell}-e\right) \rightarrow \mathfrak{q}
$$

satisfying $\pi(X)=Y$ for all $a, b, c \in Z_{0}\left(u_{\ell}-e\right) \subset Z$. Since aut $B_{0}\left(u_{\ell}-e\right)$ is a simple Lie algebra, it follows that

$$
d \Delta_{Q}\left(\left(\left\{a b^{*} z\right\}-\left\{b a^{*} z\right\}+c-\left\{z c^{*} z\right\}\right) \frac{\partial}{\partial z}\right)=0 .
$$


For $c \in Z_{0}\left(u_{\ell}-e\right)$, define

$$
q^{c}:=\exp _{B}\left(\left(c-\left\{z c^{*} z\right\}\right) \frac{\partial}{\partial z}\right) \in \exp \mathfrak{q} \subset Q .
$$

Then (3.4.67) implies

$$
\Delta_{Q}\left(q^{c}\right)=1
$$

Since

$$
q_{u_{\ell}-e}^{c}=\exp _{B_{0}\left(u_{\ell}-e\right)}\left(\left(c-\left\{\zeta c^{*} \zeta\right\}\right) \frac{\partial}{\partial \zeta}\right) \in A u t B_{0}\left(u_{\ell}-e\right),
$$

it follows that for every $q \in Q$ there is a (unique) $c \in Z_{0}\left(u_{\ell}-e\right)$ such that $q_{u_{\ell}-e}(0)=q_{u_{\ell}-e}^{c}(0)$. Then $q\left(u_{\ell}-e\right)=u_{\ell}-e+q_{u_{\ell}-e}(0)=u_{\ell}-e+q_{u_{\ell}-e}^{c}(0)=q^{c}\left(u_{\ell}-e\right)$, showing that $q=q^{c} p$ for some $p \in P$. Using (3.4.63) and Lemma 3.4.2 we obtain

$$
\begin{gathered}
\frac{\left|\operatorname{Det}_{Z} q^{\prime}\left(u_{\ell}-e\right)\right|^{2 \alpha_{\ell} / p}}{\Delta_{Q}(q)}=\frac{\left|\operatorname{Det}_{Z}\left(q^{c} p\right)^{\prime}\left(u_{\ell}-e\right)\right|^{2 \alpha_{\ell} / p}}{\Delta_{Q}\left(q^{c} p\right)}=\frac{\left|\operatorname{Det}_{Z}\left(q^{c}\right)^{\prime}\left(u_{\ell}-e\right)\right|^{2 \alpha_{\ell} / p} \Delta_{P}(p)}{\Delta_{Q}\left(q^{c}\right) \Delta_{Q}(p)} \\
=\frac{\left|\operatorname{Det}_{Z}\left(q^{c}\right)^{\prime}\left(u_{\ell}-e\right)\right|^{2 \alpha_{\ell} / p}}{\Delta_{Q}\left(q^{c}\right)}=\left|\operatorname{Det}_{Z}\left(q^{c}\right)^{\prime}\left(u_{\ell}-e\right)\right|^{2 \alpha_{\ell} / p} .
\end{gathered}
$$

For $0 \leq \theta<1$ we have $\theta\left(u_{\ell}-e\right) \in B$ and

$$
q^{c}\left(\theta\left(u_{\ell}-e\right)\right)=\exp _{B}(c-\{c k\})\left(\theta\left(u_{\ell}-e\right)\right)=\theta\left(u_{\ell}-e\right)+q_{u_{\ell}-e}^{c}(0)=\theta\left(u_{\ell}-e\right)+q_{u_{\ell}-e}(0)
$$

since $c$ and $u_{\ell}-e$ are orthogonal. This implies

$$
\Delta\left(q^{c}\left(\theta\left(u_{\ell}-e\right)\right), q^{c}\left(\theta\left(u_{\ell}-e\right)\right)\right)=\Delta\left(\theta\left(u_{\ell}-e\right), \theta\left(u_{\ell}-e\right)\right) \cdot \Delta\left(q_{u_{\ell}-e}(0), q_{u_{\ell}-e}(0)\right) .
$$

Since $\Delta\left(z_{1}, z_{2}\right)^{-p}$ is the Bergman kernel of $B$, it follows that

$$
\left|\operatorname{Det}_{Z}\left(q^{c}\right)^{\prime}\left(\theta\left(u_{\ell}-e\right)\right)\right|^{2 / p}=\frac{\Delta\left(q^{c}\left(\theta\left(u_{\ell}-e\right)\right), q^{c}\left(\theta\left(u_{\ell}-e\right)\right)\right)}{\Delta\left(\theta\left(u_{\ell}-e\right), \theta\left(u_{\ell}-e\right)\right)}=\Delta\left(q_{u_{\ell}-e}(0), q_{u_{\ell}-e}(0)\right) .
$$

Letting $\theta \rightarrow 1$, we obtain

$$
\frac{\left|\operatorname{Det}_{Z} q^{\prime}\left(u_{\ell}-e\right)\right|^{2 \alpha_{\ell} / p}}{\Delta_{Q}(q)}=\left|\operatorname{Det}_{Z}\left(q^{c}\right)^{\prime}\left(u_{\ell}-e\right)\right|^{2 \alpha_{\ell} / p}=\Delta\left(q_{u_{\ell}-e}(0), q_{u_{\ell}-e}(0)\right)^{\alpha_{\ell}} .
$$

Lemma 3.4.4 The Jacobian of $g \in G$, for the action (3.4.38) at $u_{\ell}-e \in S_{r-\ell}$ is

$$
\rho_{S_{r-\ell}}(g)=\frac{d \mu_{S_{r-\ell}}\left(\widetilde{g}\left(u_{\ell}-e\right)\right)}{d \mu_{S_{r-\ell}}\left(u_{\ell}-e\right)}=\frac{\left|\operatorname{Det}_{Z} g^{\prime}\left(u_{\ell}-e\right)\right|^{2 \alpha_{\ell} / p}}{\Delta\left(g_{u_{\ell}-e}(0), g_{u_{\ell}-e}(0)\right)^{\alpha_{\ell}}} .
$$

Proof: Write $\widetilde{g}\left(u_{\ell}-e\right)=k\left(u_{\ell}-e\right)$ for some $k \in K$. Then $\left(k^{-1} g\right)^{\sim}\left(u_{\ell}-e\right)=k^{-1} \widetilde{g}\left(u_{\ell}-e\right)=$ $u_{\ell}-e$, showing that $q:=k^{-1} g \in Q$. Since $g^{-1} K=q^{-1} K$, Proposition 3.1.2 implies

$$
\frac{d \mu_{S_{r-\ell}}\left(\widetilde{g}\left(u_{\ell}-e\right)\right)}{d \mu_{S_{r-\ell}}\left(u_{\ell}-e\right)}=\frac{d \mu_{K / K \cap Q}(g Q)}{d \mu_{K / K \cap Q}(Q)}=\Delta_{Q}(q)=\frac{\left|\operatorname{Det}_{Z} q^{\prime}\left(u_{\ell}-e\right)\right|^{2 \alpha_{\ell} / p}}{\Delta\left(q_{u_{\ell}-e}(0), q_{u_{\ell}-e}(0)\right)^{\alpha_{\ell}}} .
$$

Since $g=k q$ implies $g^{\prime}\left(u_{\ell}-e\right)=k q^{\prime}\left(u_{\ell}-e\right)$ with $\left|\operatorname{Det}_{Z} k\right|=1$, and $g_{u_{\ell}-e}(0)=k q_{u_{\ell}-e}(0)$ implies $\Delta\left(g_{u_{\ell}-e}(0), g_{u_{\ell}-e}(0)\right)=\Delta\left(k q_{u_{\ell}-e}(0), k q_{u_{\ell}-e}(0)\right)=\Delta\left(q_{u_{\ell}-e}(0), q_{u_{\ell}-e}(0)\right)$, the assertion follows. 
Lemma 3.4.5 Let $g \in G$. Then

$$
\chi(g):=\frac{\left|\operatorname{Det}_{Z} q^{\prime}\left(u_{\ell}-e+\zeta\right)\right|^{2 / p}}{\Delta\left(g_{u_{\ell}-e}(\zeta), g_{u_{\ell}-e}(\zeta)\right)} \Delta(\zeta, \zeta)
$$

is independent of $\zeta \in B_{0}\left(u_{\ell}-e\right)$.

Proof: Suppose first $g \in Q$. Write $\zeta=q_{u_{\ell}-e}(0)$ for some $q \in Q$. Then (3.4.60) implies

$$
\begin{gathered}
\frac{\left|\operatorname{Det}_{Z} g^{\prime}\left(u_{\ell}-e+\zeta\right)\right|^{2 / p}}{\Delta\left(g_{u_{\ell}-e}(\zeta), g_{u_{\ell}-e}(\zeta)\right)} \Delta(\zeta, \zeta) \Delta_{Q}(q)^{1 / \alpha_{\ell}}=\frac{\left|\operatorname{Det}_{Z} g^{\prime}\left(q\left(u_{\ell}-e\right)\right)\right|^{2 / p}\left|\operatorname{Det}_{Z} q^{\prime}\left(u_{\ell}-e\right)\right|^{2 / p}}{\Delta\left(g_{u_{\ell}-e}(\zeta), g_{u_{\ell}-e}(\zeta)\right)}= \\
\frac{\left|\operatorname{Det}_{Z}(g q)^{\prime}\left(u_{\ell}-e\right)\right|^{2 / p}}{\Delta\left((g q)_{u_{\ell}-e}(0),(g q)_{u_{\ell}-e}(0)\right)}=\Delta_{Q}(g q)^{1 / \alpha_{\ell}}=\Delta_{Q}(g)^{1 / \alpha_{\ell}} \Delta_{Q}(q)^{1 / \alpha_{\ell}} .
\end{gathered}
$$

This shows

$$
\frac{\left|\operatorname{Det}_{Z} g^{\prime}\left(u_{\ell}-e+\zeta\right)\right|^{2 / p}}{\Delta\left(g_{u_{\ell}-e}(\zeta), g_{u_{\ell}-e}(\zeta)\right)} \Delta(\zeta, \zeta)=\Delta_{Q}(g)^{1 / \alpha_{\ell}}=\frac{\left|\operatorname{Det}_{Z} g^{\prime}\left(u_{\ell}-e\right)\right|^{2 / p}}{\Delta\left(g_{u_{\ell}-e}(0), g_{u_{\ell}-e}(0)\right)}
$$

whenever $g \in Q$. If $g \in G$ then $\widetilde{g}\left(u_{\ell}-e\right)=k\left(u_{\ell}-e\right)$ for some $k \in K$. Therefore $g=k q$ for some $q \in Q$ and we obtain

$$
\begin{gathered}
\frac{\left|\operatorname{Det}_{Z} g^{\prime}\left(u_{\ell}-e+\zeta\right)\right|^{2 / p}}{\Delta\left(g_{u_{\ell}-e}(\zeta), g_{u_{\ell}-e}(\zeta)\right)} \Delta(\zeta, \zeta)=\frac{\left|\operatorname{Det}_{Z} k\right|^{2 / p}\left|\operatorname{Det}_{Z} q^{\prime}\left(u_{\ell}-e+\zeta\right)\right|^{2 / p}}{\Delta\left(q_{u_{\ell}-e}(\zeta), q_{u_{\ell}-e}(\zeta)\right)} \Delta(\zeta, \zeta) \\
=\frac{\left|\operatorname{Det}_{Z} q^{\prime}\left(u_{\ell}-e\right)\right|^{2 / p}}{\Delta\left(q_{u_{\ell}-e}(0), q_{u_{\ell}-e}(0)\right)}=\frac{\left|\operatorname{Det}_{Z} g^{\prime}\left(u_{\ell}-e\right)\right|^{2 / p}}{\Delta\left(g_{u_{\ell}-e}(0), g_{u_{\ell}-e}(0)\right)}
\end{gathered}
$$

since $\left|\operatorname{Det}_{Z} k\right|=1$ and $\Delta(k z, k z)=\Delta(z, z)$ for all $k \in K$.

Theorem 3.4.6 Define a $\mathcal{C}^{\infty}$-function $\chi_{\partial_{\ell} B}: G \rightarrow \mathbb{R}_{>}$by

$$
\chi_{\partial_{\ell} B}(g):=\left|\operatorname{Det}_{Z} g^{\prime}\left(u_{\ell}-e\right)\right|^{2 \alpha_{\ell} / p}
$$

where $\alpha_{\ell}:=\frac{n}{r}+\ell \frac{a}{2}$. Then the (well-defined) measure

$$
\mu_{\partial_{\ell} B}:=\chi_{\partial_{\ell} B} \mu_{G} / \mu_{P}
$$

on $G / P$ is $K$-invariant and gives rise to the $U_{\alpha_{\ell}}$-invariant inner product

$$
(\varphi \mid \psi)_{\alpha_{\ell}}=\int_{\partial_{\ell} B} d \mu_{\partial_{\ell} B} \bar{\varphi} \psi
$$

for $\varphi, \psi \in H_{\alpha_{\ell}}$. Moreover, there is a decomposition

$$
\int_{\partial_{\ell} B} d \mu_{\partial_{\ell} B} f=\int_{S_{r-\ell}} d v \int_{B_{0}(v)} d \mu_{B_{0}(v)}(\zeta) \Delta(\zeta, \zeta)^{\alpha_{\ell}} f(v+\zeta)
$$

for all $f \in \mathcal{C}_{c}\left(\partial_{\ell} B\right)$. Here $d v$ denotes the $K$-invariant measure on $S_{r-\ell}$, and

$$
d \mu_{B_{0}(v)}(\zeta)=d \mu_{Z_{0}(v)}(\zeta) \Delta(\zeta, \zeta)^{-(2+a(\ell-1)+b)}
$$

is the invariant measure on $B_{0}(v)$. 
Proof: For all $g \in G, p \in P$ and $k \in K$ we have

$$
\begin{aligned}
\chi_{\partial_{\ell} B}(g p) & =\left|\operatorname{Det}_{Z}(g p)^{\prime}\left(u_{\ell}-e\right)\right|^{2 \alpha_{\ell} / p}=\left|\operatorname{Det}_{Z} g^{\prime}\left(u_{\ell}-e\right)\right|^{2 \alpha_{\ell} / p}\left|\operatorname{Det}_{Z} p^{\prime}\left(u_{\ell}-e\right)\right|^{2 \alpha_{\ell} / p} \\
& =\chi_{\partial_{\ell} B}(g) \Delta_{P}(p)
\end{aligned}
$$

and

$$
\begin{aligned}
\chi_{\partial_{\ell} B}(k g) & =\left|\operatorname{Det}_{Z}(k g)^{\prime}\left(u_{\ell}-e\right)\right|^{2 \alpha_{\ell} / p}=\left|\operatorname{Det}_{Z} k\right|^{2 \alpha_{\ell} / p}\left|\operatorname{Det}_{Z} g^{\prime}\left(u_{\ell}-e\right)\right|^{2 \alpha_{\ell} / p} \\
& =\left|\operatorname{Det}_{Z} g^{\prime}\left(u_{\ell}-e\right)\right|^{2 \alpha_{\ell} / p}=\chi_{\partial_{\ell} B}(g) .
\end{aligned}
$$

Hence Proposition 3.1.1 shows that $\mu_{\partial_{\ell} B}$ is a well-defined measure on $G / P \approx \partial_{\ell} B$ which is $K$-invariant. Since (3.4.60) yields for all $q \in Q$

$$
\frac{\chi_{\partial_{\ell} B}(q)}{\Delta_{Q}(q)}=\Delta\left(q_{u_{\ell}-e}(0), q_{u_{\ell}-e}(0)\right)^{\alpha_{\ell}},
$$

Proposition 3.1.1 gives a decomposition

$$
\begin{aligned}
& \int_{\partial_{\ell} B} d \mu_{\partial_{\ell} B} f=\iint_{S_{r-\ell}} d \mu_{S_{r-\ell}}\left(k\left(u_{\ell}-e\right)\right) \int_{B_{0}\left(u_{\ell}-e\right)} d \mu_{B_{0}\left(u_{\ell}-e\right)}\left(q_{u_{\ell}-e}(0)\right) \cdot \\
& \quad \cdot \varphi\left(k\left(u_{\ell}-e+q_{u_{\ell}-e}(0)\right) \Delta\left(q_{u_{\ell}-e}(0), q_{u_{\ell}-e}(0)\right)^{\alpha_{\ell}}\right. \\
& \int_{S_{r-\ell}} d \mu_{S_{r-\ell}}\left(k\left(u_{\ell}-e\right)\right) \int_{B_{0}\left(u_{\ell}-e\right)} d \mu_{B_{0}\left(u_{\ell}-e\right)}(\zeta) \varphi\left(k\left(u_{\ell}-e+\zeta\right)\right) \Delta(\zeta, \zeta)^{\alpha_{\ell}} \\
&= \int_{S_{r-\ell}} d \mu_{S_{r-\ell}}(v) \int_{B_{0}(v)} d \mu_{B_{0}(v)}(\zeta) \varphi(v+\zeta) \Delta(\zeta, \zeta)^{\alpha_{\ell}}
\end{aligned}
$$

for all $f \in \mathcal{C}_{c}\left(\partial_{\ell} B\right)$. Now let $g, \gamma \in G$ and put $z:=\gamma\left(u_{\ell}-e\right) \in \partial_{\ell} B$. Then

$$
\begin{gathered}
\left(\frac{\ell_{g^{-1}} \chi_{\partial_{\ell} B}}{\chi_{\partial_{\ell} B}}\right)(\gamma P)=\frac{\chi_{\partial_{\ell} B}(g \gamma)}{\chi_{\partial_{\ell} B}(\gamma)}=\frac{\left|\operatorname{Det}_{Z}(g \gamma)^{\prime}\left(u_{\ell}-e\right)\right|^{2 \alpha_{\ell} / p}}{\left|\operatorname{Det}_{Z} \gamma^{\prime}\left(u_{\ell}-e\right)\right|^{2 \alpha_{\ell} / p}} \\
=\left|\operatorname{Det}_{Z} g^{\prime}\left(\gamma\left(u_{\ell}-e\right)\right)\right|^{2 \alpha_{\ell} / p}=\left|\operatorname{Det}_{Z} g^{\prime}(z)\right|^{2 \alpha_{\ell} / p} .
\end{gathered}
$$

According to [Bou63, p.57] we have for the image measure

$$
\begin{array}{r}
\int_{\partial_{\ell} B} d\left(g_{*}^{-1}\left(\mu_{\partial_{\ell} B}\right)\right) f=\int_{\partial_{\ell} B} d \mu_{\partial_{\ell} B} \ell_{g} f=\int_{\partial_{\ell} B} d\left(\frac{\chi_{\partial_{\ell} B} \mu_{G}}{\mu_{P}}\right) \ell_{g} f= \\
\int_{\partial_{\ell} B} d\left(\frac{\chi_{\partial_{\ell} B} \mu_{G}}{\mu_{P}}\right) \frac{\ell_{g^{-1}} \chi_{\partial_{\ell} B}}{\chi_{\partial_{\ell} B}} \cdot f=\int d \mu_{\partial_{\ell} B}\left|D \operatorname{Det}_{Z} g^{\prime}\right|^{2 \alpha_{\ell} / p} f .
\end{array}
$$

It follows that

$$
g_{*}^{-1}\left(\mu_{\partial_{\ell} B}\right)=\left|\operatorname{Det}_{Z} g^{\prime}\right|^{2 \alpha_{\ell} / p} \cdot \mu_{\partial_{\ell} B} .
$$

If $g \in G$ and $\varphi, \psi \in H_{\alpha_{\ell}}$, then

$$
\overline{U_{\alpha_{\ell}}(g) \varphi} U_{\alpha_{\ell}}(g) \psi=\left|\operatorname{Det}_{Z}\left(g^{-1}\right)^{\prime}\right|^{2 \alpha_{\ell} / p} \overline{\varphi \circ g^{-1}} \psi \circ g^{-1}=\left(\left|\operatorname{Det}_{Z} g^{\prime}\right|^{-2 \alpha_{\ell} / p} \bar{\varphi} \psi\right) \circ g^{-1}
$$


as functions on $\partial_{\ell} B \approx G / P$. With (3.4.93) it follows that

$$
\int_{\partial_{\ell} B} d \mu_{\partial_{\ell} B} \overline{U_{\alpha_{\ell}}(g) \varphi} U_{\alpha_{\ell}}(g) \psi=\int_{\partial_{\ell} B} d\left(g_{*}^{-1} \mu_{\partial_{\ell} B}\right)\left|\operatorname{Det}_{Z} g^{\prime}\right|^{-2 \alpha_{\ell} / p} \bar{\varphi} \psi=\int_{\partial_{\ell} B} d \mu_{\partial_{\ell} B} \bar{\varphi} \psi,
$$

showing that $\mu_{\partial_{\ell} B}$ induces the $U_{\alpha_{\ell}}$-invariant inner product on $H_{\alpha_{\ell}}$.

Remark 3.4.7 Integration formulas such as (3.4.84) were proved in [AU98, Theorem 6.3] in the somewhat simpler setting of (unbounded) Siegel domains. In the bounded case, the two extreme cases $\alpha_{0}=\frac{n}{r}$ (Hardy space) and $\alpha_{r-1}=p-1$ were also proved in [AU98].

\subsection{Restricted boundaries of symmetric domains}

Let $Z=U \oplus V$ denote the Peirce decomposition for the maximal tripotent $e=e_{1}+\cdots+e_{r}$. Let $U \ni a \mapsto R_{a} \in \operatorname{End}(V)$ denote the canonical representation of $U$ on $V$. The "Cayley transform"

$$
c(u, v):=\left((e+u) \circ(e-u)^{-1}, \sqrt{2} R_{(e-u)^{-1}} v\right)
$$

defines a biholomorphic mapping $c: B \rightarrow D$, with inverse

$$
c^{-1}(u, v):=\left((u-e) \circ(u+e)^{-1}, \frac{1}{\sqrt{2}} \cdot R_{(u+e)^{-1}} v\right) .
$$

Note that $c(0)=e$ and $c(-e)=0$. Since $\operatorname{Aff}(D) \subset \operatorname{Aut}(D)$ is the stabilizer of $c(e)$ (in the compact dual of $B$ ), it follows that

$$
G_{e}:=\{g \in G: g(e)=e\}=c^{-1} \operatorname{Aff}(D) c=G_{e}^{+} G_{e}^{0},
$$

where $G_{e}^{+}:=c^{-1} \Sigma c, G_{e}^{0}:=c^{-1} G L(D) c$.

Proposition 3.5.1 If $g \in G_{e}$ then $\left.g^{\prime}(e)\right|_{X} \in G L(\Omega)$ and

$$
\tau\left(\left(c g^{-1} c^{-1}\right)(x)\right)=\left.g^{\prime}(e)\right|_{X} ^{*} x
$$

for all $x \in \Omega$, where $h \mapsto h^{*}$ is the involution in $G L(\Omega)$. Moreover

$$
\left|\operatorname{Det}_{Z}\left(c g c^{-1}\right)^{\prime}(w)\right|=\Delta\left(g^{\prime}(e) e\right)^{-p / 2}
$$

for all $w \in \bar{D}$.

Proof: Let $t_{a, b} \in \Sigma$ and put $t:=c^{-1} t_{a, b} c \in G_{e}$. Since

$$
t_{a, b}^{\prime}(u, v)(\dot{u}, \dot{v})=(\dot{u}+2 \Phi(\dot{v}, b), \dot{v})
$$

for all $w=(u, v) \in \bar{D}$ and $(\dot{u}, \dot{v}) \in Z$, we obtain

$$
\operatorname{Det}_{Z}\left(c t c^{-1}\right)^{\prime}(w)=\operatorname{Det}_{Z} t_{a, b}^{\prime}(w)=\operatorname{Det}_{Z}\left[\begin{array}{cc}
i d_{U} & 2 \Phi(-, b) \\
0 & i d_{V}
\end{array}\right]=1 .
$$


Moreover $t_{a, b}^{\prime}(w) x=x$ for all $x \in X$. If $0 \leq \theta<1$, then $c(\theta e) \in \bar{D}$ and $c^{\prime}(\theta e) x \in X$. Therefore

$$
c^{\prime}(t(\theta e)) t^{\prime}(\theta e) x=(c t)^{\prime}(\theta e) x=\left(t_{a, b} c\right)^{\prime}(\theta e) x=t_{a, b}^{\prime}(c(\theta e)) c^{\prime}(\theta e) x=c^{\prime}(\theta e) x .
$$

Letting $\theta \rightarrow 1$ and using $t(e)=e$, we obtain

$$
t^{\prime}(e) x=x
$$

for all $x \in X$. Now let $h \in G L(D)$. The commuting diagram

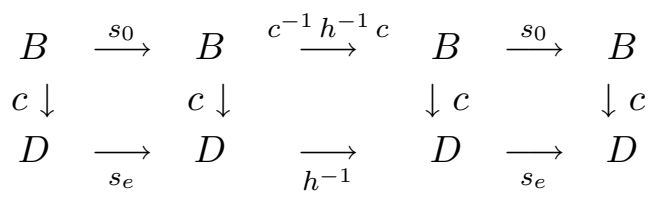

for the symmetries $s_{0}=-i d_{B}$ and $s_{e}$ around $0 \in B$ and $e \in D$, resp., shows that

$$
\begin{aligned}
& h^{*}=\left(s_{e} h^{-1} s_{e}\right)^{\prime}(0)=\left(c s_{0}\left(c^{-1} h^{-1} c\right) s_{0} c^{-1}\right)^{\prime}(0)= \\
& \quad c^{\prime}(-e) s_{0}^{\prime}(e)\left(c^{-1} h^{-1} c\right)^{\prime}(e) s_{0}^{\prime}(-e)\left(c^{-1}\right)^{\prime}(0)=\left(c^{-1} h^{-1} c\right)^{\prime}(e)
\end{aligned}
$$

since $c^{\prime}(-e)=\left(c^{-1}\right)^{\prime}(0)$ is scalar and $s_{0}^{\prime}(e)=s_{0}^{\prime}(-e)=-i d$. Since

$$
\left|\operatorname{Det}_{Z} h\right|=\Delta(h e)^{p / 2}
$$

we obtain for all $w \in \bar{D}$

$$
\begin{aligned}
& \left|\operatorname{Det}_{Z} h^{\prime}(w)\right|=\left|\operatorname{Det}_{Z} h\right|=\left|\operatorname{Det}_{Z}\left(c^{-1} h^{-1} c\right)^{\prime}(e)^{*}\right|= \\
& \Delta\left(\left(c^{-1} h^{-1} c\right)^{\prime}(e)^{*} e\right)^{p / 2}=\Delta\left(\left(c^{-1} h c\right)^{\prime}(e)^{*} e\right)^{-p / 2}=\Delta\left(\left(c^{-1} h c\right)^{\prime}(e) e\right)^{-p / 2} .
\end{aligned}
$$

Now let $g=t\left(c^{-1} h c\right)$ with $c t c^{-1} \in \Sigma$ and $h \in G L(D)$. Then

$$
\left(g^{-1}\right)^{\prime}(e) x=\left(c^{-1} h^{-1} c\right)^{\prime}(e)\left(t^{-1}\right)^{\prime}(e) x=\left(c^{-1} h^{-1} c\right)^{\prime}(e) x=h_{1}^{*} x
$$

showing that

$$
\left.\left(g^{-1}\right)^{\prime}(e)\right|_{X} ^{*}=h_{1}
$$

On the other hand,

$$
\tau\left(\left(c g c^{-1}\right)(x)\right)=\tau\left(\operatorname{ctc} c^{-1}(h x)\right)=h_{1} x .
$$

This proves (3.5.4). Now let $w \in \bar{D}$. Then $h w \in \bar{D}$ and

$$
\begin{aligned}
& \left|\operatorname{Det}_{Z}\left(\operatorname{cgc}^{-1}\right)^{\prime}(w)\right|=\left|\operatorname{Det}_{Z}\left(\operatorname{ctc}^{-1}\right)^{\prime}(h w)\right| \cdot\left|\operatorname{Det}_{Z} h^{\prime}(w)\right|=\left|\operatorname{Det}_{Z} h^{\prime}(w)\right| \\
& \quad=\Delta\left(\left(c^{-1} h c\right)^{\prime}(e) e\right)^{-p / 2}=\Delta\left(g^{\prime}(e) e\right)^{-p / 2} .
\end{aligned}
$$

Now (3.5.5) follows since (3.5.9) implies

$$
g^{\prime}(e) e=t^{\prime}(e)\left(c^{-1} h c\right)^{\prime}(e) e=\left(c^{-1} h c\right)^{\prime}(e) e .
$$

Proposition 3.5.2 $G_{e}$ has the modulus function

$$
\Delta_{G_{e}}(g)=\Delta\left(g^{\prime}(e) e\right)^{n / r} .
$$


Proof: By Corollary 3.3.2 and Proposition 3.5.1

$$
\begin{aligned}
& \Delta_{G_{e}}(g)=\Delta_{A f f(D)}\left(c g c^{-1}\right)=\Delta\left(\tau\left(\left(c g c^{-1}\right)(e)\right)^{-n / r}\right. \\
& \quad=\Delta\left(\left(g^{-1}\right)^{\prime}(e)^{*} e\right)^{-n / r}=\Delta\left(g^{\prime}(e)^{*} e\right)^{n / r}=\Delta\left(g^{\prime}(e) e\right)^{n / r} .
\end{aligned}
$$

Proposition 3.5.3 NA has the modulus function

$$
\Delta_{N A}(g)=\Delta_{-2 \rho}\left(g^{\prime}(e)^{*} e\right)
$$

where $2 \rho_{k}=a(k-1)+1+b$.

Proof: Since $N A \subset G_{e}$ and $c N A c^{-1}=N_{D} A_{D}$, we may apply Proposition 3.3.3 and Proposition 3.5.7 and obtain

$$
\Delta_{N A}(g)=\Delta_{N_{D} A_{D}}\left(c g c^{-1}\right)=\Delta_{-2 \rho}\left(\tau\left(c g c^{-1}(e)\right)\right)=\Delta_{-2 \rho}\left(g^{\prime}(e)^{*} e\right) .
$$

Lemma 3.5.4 Let $z \in \bar{B}$ satisfy $\Delta(e-z) \neq 0$. Then $c(z) \in \bar{D} \subset Z$ and $\operatorname{Det}_{Z} c^{\prime}(z)=2^{r p / 2} \Delta(e-z)^{-p}$.

Proof: The derivative at $z=(u, v)$ is given by

$$
c^{\prime}(z)(\dot{u}, \dot{v})=\left(2 P_{e-u}^{-1} \dot{u}, \quad \sqrt{2}\left(R_{(e-u)^{-1}} \dot{v}+R_{P_{e-u}^{-1} \dot{u}} v\right)\right) .
$$

Being of "block-triangular" form, $c^{\prime}(z)$ has determinant

$$
\begin{aligned}
\operatorname{Det}_{Z} c^{\prime}(z) & =\operatorname{Det}_{U} 2 P_{e-u}^{-1} \cdot \operatorname{Det}_{V} \sqrt{2} R_{(e-u)^{-1}} \\
& =2^{\operatorname{dim} U+\operatorname{dim} V / 2} \operatorname{Det}_{Z} \mathbf{R}_{(e-u)^{-1}}
\end{aligned}
$$

Since $\mathbf{R}_{(e-u)^{-1}}:=\left(P_{e-u}^{-1}, \mathbf{R}_{(e-u)^{-1}}\right) \in G L(D)$, we have

$$
\operatorname{Det}_{Z} \mathbf{R}_{(e-u)^{-1}}=\Delta\left(\mathbf{R}_{(e-u)^{-1}} e\right)^{p / 2}=\Delta(e-u)^{-p}=\Delta(e-z)^{-p} .
$$

Now the assertion follows, since

$$
\operatorname{dim} U+\frac{1}{2} \operatorname{dim} V=r+\frac{r(r-1)}{2} a+\frac{r b}{2}=\frac{r p}{2} .
$$

Corollary 3.5.5 If $\Delta(e-z) \neq 0$, then

$$
\Delta(\tau(c(z)))=\frac{\Delta(z, z)}{|\Delta(e-z)|^{2}} .
$$


Proof: Since $c: B \rightarrow D$ is biholomorphic, we have

$$
\left|\operatorname{Det}_{Z} c^{\prime}(z)\right|^{2} K_{D}(c(z), c(z))=K_{B}(z, z)
$$

for all $z \in B$, where $K_{B}$ and $K_{D}$ denote the respective Bergman kernels. This implies

$$
a \frac{\Delta(\tau(c(z)))^{p}}{\Delta(z, z)^{p}}=\left|\operatorname{Det}_{Z} c^{\prime}(z)\right|^{2}=2^{r p}|\Delta(e-z)|^{-2 p}
$$

where $a$ is a fixed constant. Evaluating at $z=0$ yields $a=2^{r p}$.

Now fix $0 \leq \ell<r$ and put $u_{\ell}-e=-e_{\ell+1}-\cdots-e_{r}$. Then the "restricted" boundary orbit

$$
\partial_{\ell}^{\prime} B=\left\{z \in \partial_{\ell} B: \Delta(e-z) \neq 0\right\}=c^{-1}\left(\partial_{\ell} D\right)=G_{e}\left(u_{\ell}-e\right)
$$

is an open dense subset of $\partial_{\ell} B$. We may identify

$$
G_{e} / P_{e} \ni g P_{e} \mapsto g\left(u_{\ell}-e\right) \in \partial_{\ell}^{\prime} B
$$

where

$$
P_{e}=G_{e} \cap P \subset G_{e}^{o}
$$

In terms of the fibration (3.4.37), we put

$$
S_{r-\ell}^{\prime}:=\left\{v \in S_{r-\ell}: \Delta(e-v) \neq 0\right\}=\left\{v \in S_{r-\ell}: v+B_{0}(v) \subset \partial_{\ell}^{\prime} B\right\}
$$

as a dense open subset of $S_{r-\ell}$, which is $K_{e}$-invariant and contains $\Pi_{\ell}-e$. Note that $S_{r-\ell}^{\prime} \cap \Pi_{r-\ell}=\emptyset$. By definition, $S_{r-\ell}^{\prime}$ is a $G_{e^{-}}$orbit for the action (3.4.38), i.e. there is a diffeomorphism

$$
G_{e} / Q_{e} \ni g \mapsto \widetilde{g}\left(u_{\ell}-e\right) \in S_{r-\ell}^{\prime},
$$

where

$$
Q_{e}=G_{e} \cap Q=Q_{e}^{+} Q_{e}^{o}
$$

is a closed subgroup of $G_{e}$ and we put

$$
Q_{e}^{+}:=Q \cap G_{e}^{+}, \quad Q_{e}^{o}:=Q \cap G_{e}^{0} .
$$

Theorem 3.5.6 Under the mapping $c^{-1}: \partial_{\ell} D \rightarrow \partial_{\ell}^{\prime} B$, the measure $\mu_{\partial_{\ell} D}$ constructed in (3.3.38) has the image measure

$$
\mu_{\partial_{\ell}^{\prime} B}:=c_{*}^{-1}\left(\mu_{\partial_{\ell} D}\right)
$$

on $\partial_{\ell}^{\prime} B$, which is relatively invariant under $G_{e}$ with multiplier

$$
\chi_{\partial_{\ell}^{\prime} B}(g):=\Delta\left(g^{\prime}(e) e\right)^{-\alpha_{\ell}}
$$

for all $g \in G_{e}$. Moreover, $\mu_{\partial_{\ell}^{\prime} B}$ has a decomposition

$$
\int_{\partial_{\ell}^{\prime} B} d \mu_{\partial_{\ell}^{\prime} B} f=\int_{\Pi_{\ell}} d u \int_{\Sigma / \Sigma_{1}(u)} d \dot{t} \int_{B_{0}(u-e)} d \mu_{B_{0}(u-e)}(\zeta) \frac{\Delta(\zeta, \zeta)^{\alpha_{\ell}}}{|\Delta(e-\zeta)|^{2 \alpha_{\ell}}} f\left(t^{c}(\zeta+u-e)\right)
$$

for all $f \in \mathcal{C}_{c}\left(\partial_{\ell}^{\prime} B\right)$, where $t^{c}:=c^{-1} t c \in G_{e}^{+}$and

$$
d \mu_{B_{0}(u-e)}(\zeta)=2^{\ell p_{\ell}} d \mu_{Z_{0}(u-e)}(\zeta) \Delta(\zeta, \zeta)^{-p_{\ell}}
$$

is the invariant measure on $B_{0}(u-e)$, with $p_{\ell}:=2+a(\ell-1)+b$. 
Proof: For $g \in G_{e}$ we have $c g c^{-1} \in A f f(D)$, and Theorem 3.3.4 and Proposition 3.5.1 imply

$$
\begin{array}{r}
g_{*}\left(\mu_{\partial_{\ell}^{\prime} B}\right)=c_{*}^{-1}\left(c g c^{-1}\right)_{*}\left(c_{*}\left(\mu_{\partial_{\ell}^{\prime} B}\right)\right)=c_{*}^{-1}\left(c g c^{-1}\right)_{*}\left(\mu_{\partial_{\ell} D}\right)= \\
\Delta\left(\tau\left(c g^{-1} c^{-1}(e)\right)\right)^{\alpha_{\ell}} c_{*}^{-1}\left(\mu_{\partial_{\ell} D}\right)=\Delta\left(g^{\prime}(e)^{*} e\right)^{\alpha_{\ell}} \mu_{\partial_{\ell}^{\prime} B}=\Delta\left(g^{\prime}(e) e\right)^{\alpha_{\ell}} \mu_{\partial_{\ell}^{\prime} B} .
\end{array}
$$

This shows that $\mu_{\partial_{\ell}^{\prime} B}$ is relatively invariant under $G_{e}$, with multiplier given by (3.5.37). For any $u \in \Pi_{\ell}$, let $c_{u}: B_{0}(u-e) \rightarrow D_{1}(u)$ denote the "partial" Cayley transform relative to $u \in Z_{0}(u-e)$. Then

$$
c(\zeta+u-e)=c_{u}(\zeta), c_{u}(\zeta)+e-u=c(\zeta)
$$

for all $\zeta \in B_{0}(u-e)$. Moreover

$$
\left(c_{u}\right)_{*}\left(\mu_{B_{0}(u-e)}\right)=\mu_{D_{1}(u)}
$$

for the respective invariant measures. Applying Theorem 3.3.4, it follows that

$$
\begin{aligned}
& \int_{\partial_{\ell}^{\prime} B} d \mu_{\partial_{\ell}^{\prime} B} f=\int_{\partial_{\ell} D} d \mu_{\partial_{\ell} D}(w) f\left(c^{-1}(w)\right)= \\
& \int_{\Pi_{\ell}} d u \int_{\Sigma / \Sigma_{1}(u)} d \dot{t} \int_{D_{1}(u)} d \mu_{D_{1}(u)}(\omega) \Delta(\tau(\omega+e-u))^{\alpha \ell} \varphi\left(c^{-1}(t \omega)\right)= \\
& \int_{\Pi_{\ell}} d u \int_{\Sigma / \Sigma_{1}(u)} d \dot{t} \int_{B_{0}(u-e)} d \mu_{B_{0}(u-e)}(\zeta) \Delta\left(\tau\left(c_{u}(\zeta)+e-u\right)\right)^{\alpha} \varphi\left(c^{-1}\left(t c_{u}(\zeta)\right)\right) .
\end{aligned}
$$

Now the assertion follows from the identities

$$
\begin{gathered}
\Delta\left(\tau\left(c_{u}(\zeta)+e-u\right)\right)=\Delta(\tau(c(\zeta)))=\frac{\Delta(\zeta, \zeta)}{|\Delta(\zeta-e)|^{2}}, \\
c^{-1}\left(t c_{u}(\zeta)\right)=c^{-1}(t c(\zeta+u-e))=t^{c}(\zeta+u-e) .
\end{gathered}
$$

We will now express the relationship between the relatively invariant measure $\mu_{\partial_{\ell}^{\prime} B}$ on $\partial_{\ell}^{\prime} B$, and the $K$-invariant measure $\mu_{\partial_{\ell} B}$ on $\partial_{\ell} B$ constructed in Theorem 3.4.6, giving rise to the invariant inner product for parameter $\alpha_{\ell}$.

Proposition 3.5.7 On $\partial_{\ell}^{\prime} B \subset \partial_{\ell} B$ we have

$$
|\Delta(e-z)|^{2 \alpha_{\ell}} \cdot d \mu_{\partial_{\ell}^{\prime} B}(z)=2^{r \alpha_{\ell}} \cdot d \mu_{\partial_{\ell} B}(z) .
$$

Proof: By Proposition 3.5.1, we have for all $g \in G_{e}$

$$
\begin{aligned}
\left|\operatorname{Det}_{Z} c^{\prime}(g(z)) \operatorname{Det}_{Z} g^{\prime}(z)\right|^{2 \alpha_{\ell} / p} & =\left|\operatorname{Det}_{Z}(c g)^{\prime}(z)\right|^{2 \alpha_{\ell} / p}= \\
\left|\operatorname{Det}_{Z}\left(c g c^{-1}\right)^{\prime}(c(z)) \operatorname{Det}_{Z} c^{\prime}(z)\right|^{2 \alpha_{\ell} / p} & =\chi_{\partial_{\ell}^{\prime} B}(g) \cdot\left|\operatorname{Det}_{Z} c^{\prime}(z)\right|^{2 \alpha_{\ell} / p} .
\end{aligned}
$$

Using (3.4.93) this implies

$$
\begin{aligned}
& g_{*}^{-1}\left(\left|\operatorname{Det}_{Z} c^{\prime}\right|^{2 \alpha_{\ell} / p} \mu_{\partial_{\ell} B}\right)=\left|\operatorname{Det}_{Z} c^{\prime} \circ g\right|^{2 \alpha_{\ell} / p} g_{*}^{-1}\left(\mu_{\partial_{\ell} B}\right)= \\
& \mid \text { Det }\left._{Z} c^{\prime} \circ g\right|^{2 \alpha_{\ell} / p}\left|\operatorname{Det}_{Z} g^{\prime}\right|^{2 \alpha_{\ell} / p} \mu_{\partial_{\ell} B}=\chi_{\partial_{\ell}^{\prime} B}(g)\left|\operatorname{Det}_{Z} c^{\prime}\right|^{2 \alpha_{\ell} / p} \mu_{\partial_{\ell} B} .
\end{aligned}
$$


This shows that $\left|\operatorname{Det}_{Z} c^{\prime}\right|^{2 \alpha_{\ell} / p} \mu_{\partial_{\ell} B}$ is relatively invariant under $G_{e}$, with multiplier $\chi_{\partial_{\ell}^{\prime} B}$. Using Lemma 3.5.4, we obtain, up to a constant factor,

$$
d \mu_{\partial_{\ell}^{\prime} B}(z)=\left|\operatorname{Det}_{Z} c^{\prime}(z)\right|^{2 \alpha_{\ell} / p} d \mu_{\partial_{\ell} B}(z)=2^{r \alpha_{\ell}}|\Delta(e-z)|^{-2 \alpha_{\ell}} d \mu_{\partial_{\ell} B}(z) .
$$

Theorem 3.5.8 For $0 \leq \ell<r$ and $f \in \mathcal{C}_{c}\left(S_{r-\ell}^{\prime}\right)$, we have

$$
2^{r \alpha_{\ell}} \int_{S_{r-\ell}^{\prime}} d v f(v)=\int_{\Pi_{\ell}} d u \int_{\Sigma / \Sigma_{1}(u)} d \dot{t} f\left(\widetilde{t}^{c}(u-e)\right) \frac{\left|\Delta\left(e-t^{c}(u-e)\right)\right|^{2 \alpha_{\ell}}}{\Delta\left(t_{u-e}^{c}(0), t_{u-e}^{c}(0)\right)^{\alpha_{\ell}}} .
$$

Here we put $t^{c}:=c^{-1} t c$ for $t \in \Sigma$, and decompose $t^{c}\left(u_{\ell}-e\right)=\tilde{t}^{c}\left(u_{\ell}-e\right)+t_{u_{\ell}-e}^{c}(0)$ according to (3.4.56).

Proof: By (3.5.7), $\operatorname{Det}_{Z} t^{\prime}(w)=1$ for all $t \in \Sigma$. Therefore Lemma 3.5.4 implies for all $u \in \Pi_{\ell}$ and $\zeta \in B_{0}(u-e)$

$$
\begin{aligned}
\operatorname{Det}_{Z} & \left(t^{c}\right)^{\prime}(u-e+\zeta)=\operatorname{Det}_{Z}\left(c^{-1} t c\right)^{\prime}(u-e+\zeta) \\
& =\operatorname{Det}_{Z}\left(c^{-1}\right)^{\prime}\left(t(c(u-e+\zeta)) \operatorname{Det}_{Z} c^{\prime}(u-e+\zeta)\right. \\
& =\operatorname{Det}_{Z} c^{\prime}\left(t^{c}(u-e+\zeta)\right)^{-1} \operatorname{Det}_{Z} c^{\prime}(u-e+\zeta) \\
& =\Delta\left(e-t^{c}(u-e+\zeta)\right)^{p} \Delta(e-(u-e+\zeta))^{-p} \\
& =2^{p(\ell-r)} \Delta\left(e-t^{c}(u-e+\zeta)\right)^{p} \Delta(e-\zeta)^{-p},
\end{aligned}
$$

since $\Delta(e-(u-e+\zeta))=2^{r-\ell} \Delta(e-\zeta)$. Applying Lemma 3.4.5 to $t^{c}$ and setting $\zeta=0$, we obtain

$$
\begin{aligned}
& \frac{\Delta(\zeta, \zeta)}{\Delta\left(t_{u-e}^{c}(\zeta), t_{u-e}^{c}(\zeta)\right)} \frac{\left|\Delta\left(e-t^{c}(u-e+\zeta)\right)\right|^{2}}{|\Delta(e-\zeta)|^{2}}= \\
& 4^{r-\ell} \frac{\Delta(\zeta, \zeta)}{\Delta\left(t_{u-e}^{c}(\zeta), t_{u-e}^{c}(\zeta)\right)}\left|\operatorname{Det}_{Z}\left(t^{c}\right)^{\prime}(u-e+\zeta)\right|^{2 / p}=\frac{\left|\Delta\left(e-t^{c}(u-e)\right)\right|^{2}}{\mid \Delta\left(t_{u-e}^{c}(0), t_{u-e}^{c}(0)\right)}
\end{aligned}
$$

Since $\alpha_{\ell}-p_{\ell}=\frac{a}{2}(r-\ell+1)-1 \geq a-1 \geq 0$, there exists a constant $c$ such that

$$
\int_{B_{0}(v)} d \mu_{B_{0}(v)}(w) \Delta(w, w)^{\alpha_{\ell}}=c
$$

for all $v \in S_{r-\ell}$. Since $t_{u-e}^{c}: B_{0}(u-e) \rightarrow B_{0}\left(\widetilde{t}^{c}(u-e)\right)$ is biholomorphic, we have

$$
\left(t_{u-e}^{c}\right)_{*}\left(\mu_{B_{0}(u-e)}\right)=\mu_{B_{0}\left(\widetilde{t^{c}}(u-e)\right)}
$$

and hence

$$
\int_{B_{0}(u-e)} d \mu_{B_{0}(u-e)}(\zeta) \Delta\left(t_{u-e}^{c}(\zeta), t_{u-e}^{c}(\zeta)\right)^{\alpha_{\ell}}=c .
$$

Applying Theorem 3.4.6, Proposition 3.5.7 and Theorem 3.5.6, we obtain for $f \in \mathcal{C}_{c}\left(S_{r-\ell}^{\prime}\right)$ 


$$
\begin{aligned}
c 2^{r \alpha_{\ell}} & \int_{S_{r-\ell}^{\prime}} d v f(v)=2^{r \alpha_{\ell}} \int_{S_{r-\ell}^{\prime}} d v \int_{B_{0}(v)} d \mu_{B_{0}(v)}(w) f(v) \Delta(w, w)^{\alpha_{\ell}} \\
= & 2^{r \alpha_{\ell}} \int_{\partial_{\ell}^{\prime} B} d \mu_{\partial_{\ell} B}(v+w) f(v)=\int_{\partial_{\ell}^{\prime} B} d \mu_{\partial_{\ell}^{\prime} B}(v+w) f(v)|\Delta(e-(v+w))|^{2 \alpha_{\ell}} \\
= & \int_{\Pi_{\ell}} d u \int_{\Sigma / \Sigma_{1}(u)} d \dot{t} f\left(\widetilde{t}^{c}(u-e)\right) \int_{B_{0}(u-e)} d \mu_{B_{0}(u-e)}(\zeta) \frac{\Delta(\zeta, \zeta)^{\alpha_{\ell}}}{|\Delta(e-\zeta)|^{2 \alpha_{\ell}}}\left|\Delta\left(e-t^{c}(u-e+\zeta)\right)\right|^{2 \alpha_{\ell}} \\
= & \int_{\Pi_{\ell}} d u \int_{\Sigma / \Sigma_{1}(u)} d \dot{t} f\left(\widetilde{t}^{c}(u-e)\right) \frac{\left|\Delta\left(e-t^{c}(u-e)\right)\right|^{2 \alpha_{\ell}}}{\Delta\left(t_{u-e}^{c}(0), t_{u-e}^{c}(0)\right)^{\alpha_{\ell}}} \cdot \int_{B_{0}(u-e)} d \mu_{B_{0}(u-e)}(\zeta) \Delta\left(t_{u-e}^{c}(\zeta), t_{u-e}^{c}(\zeta)\right)^{\alpha_{\ell}} \\
& c \int_{\Pi_{\ell}} d u \int_{\Sigma / \Sigma_{1}(u)} d \dot{t} f\left(\widetilde{t}^{c}(u-e)\right) \frac{\left|\Delta\left(e-t^{c}(u-e)\right)\right|^{2 \alpha_{\ell}}}{\Delta\left(t_{u-e}^{c}(0), t_{u-e}^{c}(0)\right)^{\alpha_{\ell}}} .
\end{aligned}
$$

Example 3.5.9 For $\ell=0, S_{r}=S$ is the Shilov boundary. If $B$ is of tube type, we have

$$
S_{r}^{\prime}=c^{-1}(i X)
$$

For $t \in \Sigma$ set $a=t(0)$. Then

$$
t^{c}(-e)=c^{-1} t c(-e)=c^{-1} t(0)=c^{-1}(a)=(a-e) \circ(a+e)^{-1}
$$

and hence $e-t^{c}(-e)=2(a+e)^{-1}$. It follows that

$$
\left|\Delta\left(e-t^{c}(-e)\right)\right|=2^{r}|\Delta(a+e)|^{-1}=2^{r} \Delta\left(e-a^{2}\right)^{-1 / 2} .
$$

Theorem 3.5.8 yields for all $f \in \mathcal{C}_{c}\left(S^{\prime}\right)$

$$
2^{n} \int_{S^{\prime}} d v f(v)=\int_{\Sigma} d t f\left(t^{c}(-e)\right)\left|\Delta\left(e-t^{c}(-e)\right)\right|^{2 n / r}=4^{n} \int_{i X} d a f\left(c^{-1}(a)\right) \Delta\left(e-a^{2}\right)^{-n / r}
$$

i.e. we obtain the well-known formula [FK94]

$$
\int_{S^{\prime}} d v f(v)=2^{n} \int_{i X} d a f\left(c^{-1}(a)\right) \Delta\left(e-a^{2}\right)^{-n / r} .
$$

\section{References}

[A92-1] J. Arazy, Realization of the invariant inner products on the highest quotients of the composition series, Arkiv Mat. 30 (1992), 1-24. 
[A92-2] J. Arazy, Integral formulas for the invariant inner products in spaces of analytic functions in the unit ball, Lect. Notes in Pure and Applied Math., Vol.136, Marcel Dekker (1992), 9-23.

[A95] J. Arazy, A survey of invariant Hilbert spaces of analytic functions on bounded symmetric domains, Contemp. Math. 185 (1995), 7-65.

[A96] J. Arazy, Boundedness and compactness of generalized Hankel operators on bounded symmetric domains J. Funct. Anal. 137 (1996), 97-151.

[AU97] J. Arazy and H. Upmeier, Invariant inner products in spaces of holomorphic functions on bounded symmetric domains, Documenta Math. 2 (1997), 213-261.

[AU98] J. Arazy and H. Upmeier, Discrete series representations and integration over boundary orbits of symmetric domains, Contemp. Math. 214 (1998), 1-22.

[Be75] F. Berezin, Quantization in complex symmetric spaces, Math. USSR-Izv. 9 (1975), 341-379.

[Bou63] N. Bourbaki, Éléments de Mathématique, Livre VI, Intégration, Chap. 7 \& 8, Herman (1963).

[Dieu74] J. Dieudonné, Treatise on Analysis, vol. IV, Academic Press, 1974.

[FK90] J. Faraut and A. Korányi, Function spaces and reproducing kernels on bounded symmetric domains, J. Funct. Anal. 88 (1990), 64-89.

[FK94] J. Faraut and A. Korányi, Analysis on Symmetric Cones, Clarendon Press, Oxford (1994).

[Gi64] S. Gindikin, Analysis on homogeneous domains, Russ. Math. Surv. 19 (1964), 1-89.

[Gi75] S. Gindikin, Invariant generalized functions in homogeneous domains, Funct. Anal. Appl. 9 (1975), 50-52.

[Ga47] L. Gårding, The solution of Cauchy's problem for two totally hyperbolic linear differential equations by means of Riesz integrals, Ann. Math. 48 (1947), 785-826.

[He78] S. Helgason, Differential Geometry, Lie Groups, and Symmetric spaces, Academic Press (1978).

[He84] S. Helgason, Groups and Geometric Analysis, Academic Press (1984).

[Hu63] L.K. Hua, Harmonic Analysis of Functions of Several Complex Variables in the Classical Domains, Transl. Math. Monographs, Amer. Math. Soc. (1963).

[La86] M. Lassalle, Noyau de Szegö, K-types et algèbres de Jordan, C. R. Acad. Sci. Paris 303 (1986), 1-4.

[La87] M. Lassalle, Algèbres de Jordan et ensemble de Wallach, Invent. Math. 89 (1987), 375-393. 
[Lo75] O. Loos, Jordan Pairs, Springer Lect. Notes in Math. 460 (1975).

[Lo77] O. Loos, Bounded Symmetric Domains and Jordan Pairs, Univ. of California, Irvine (1977).

[M87] I. G. Macdonald, Commuting differential operators and zonal spherical functions, Springer Lect. Notes in Math. 1271 (1987), 189-200.

[M95] I. G. Macdonald, Symmetric Functions and Hall Polynomials, Clarendon Press, Oxford (1995).

[N89] T. Nomura, Algebraically independent generators of invariant differential operators on a symmetric cone, J. reine angew. Math. 400 (1989), 122-133.

[O80] B. Orsted, Composition series for analytic continuations of holomorphic discrete series representations of $S U(n, n)$, Trans. Amer. Math. Soc. 260 (1980), 563-573.

[Ri49] M. Riesz, L'intégrale de Riemann-Liouville et le problème de Cauchy, Acta Math. 81 (1949), 1-223.

[RV76] H. Rossi and M. Vergne, Analytic continuation of holomorphic discrete series of a semi-simple Lie group, Acta Math. 136 (1975), 1-59.

[Sch69] W. Schmid, Die Randwerte holomorpher Funktionen auf hermitesch symmetrischen Räumen, Invent. Math. 8 (1969), 61-80.

[St89] R. P. Stanley, Some combinatorial properties of the Jack symmetric functions, Adv. Math. 77 (1989), 76-115.

[UU94] A. Unterberger and H. Upmeier, Berezin transform and invariant differential operators, Comm. Math. Phys. 164 (1994), 563-597.

[U83] H. Upmeier, Toeplitz operators on bounded symmetric domains, Trans. Amer. Math. Soc. 280 (1983), 221-237.

[U85] H. Upmeier, Toeplitz operators on symmetric Siegel domains, Math. Ann. 271 (1985), 401-414.

[U85a] H. Upmeier, Symmetric Banach Manifolds and Jordan $C^{*}$-Algebras, North Holland 1985 .

[U86] H. Upmeier, Jordan algebras and harmonic analysis on symmetric spaces, Amer. J. Math. 108 (1986), 1-25.

[U87] H. Upmeier, Jordan Algebras in Analysis, Operator Theory, and Quantum Mechanics, CBMS Series in Math. 67, Amer. Math. Soc. (1987).

[W79] N. Wallach, The analytic continuation of the discrete series, I, II, Trans. Amer. Math. Soc. 251 (1979), 1-17 and 19-37.

[Y93] Z. Yan, Differential operators and function spaces, Contemp. Math. 142 (1993), 121142 . 


\section{Authors' Addresses:}

Jonathan Arazy: Department of Mathematics, University of Haifa, Haifa 31905, Israel.

Electronic Address: jarazy@math.haifa.ac.il

Harald Upmeier: Fachbereich Mathematik, Universität Marburg, D-35032 Marburg, Germany.

Electronic Address: upmeier@mathematik.uni-marburg.de 